Supporting Information for:

\title{
Modulating the Optical Properties of BODIPY Dyes by Noncovalent Dimerization within a Flexible Coordination Cage
}

Julius Gemen, ${ }^{\dagger}$ Johannes Ahrens, ${ }^{\dagger,}{ }^{\ddagger}$ Linda J. W. Shimon, ${ }^{\curvearrowleft}$ Rafal Klajn ${ }^{\dagger}$

†Department of Organic Chemistry, Weizmann Institute of Science, Rehovot 76100, Israel

‡BASF SE, Carl-Bosch-Straße 38, Ludwigshafen am Rhein 67056, Germany

ॠChemical Research Support, Weizmann Institute of Science, Rehovot 76100, Israel

\section{Table of contents}

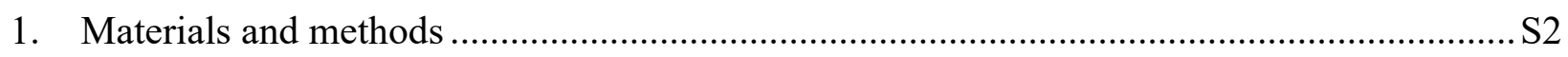

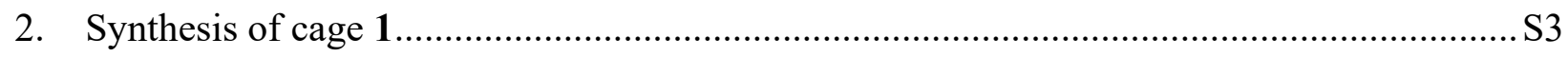

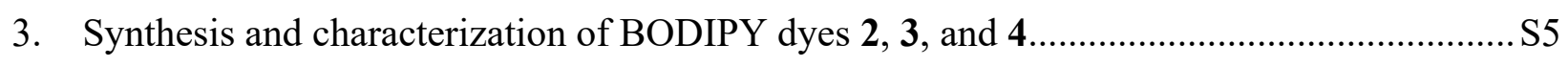

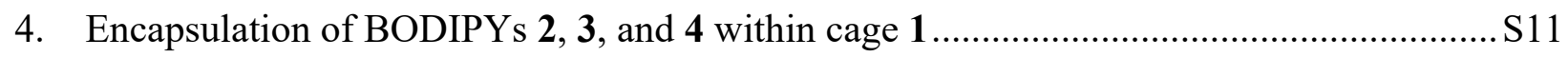

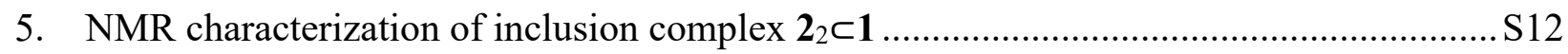

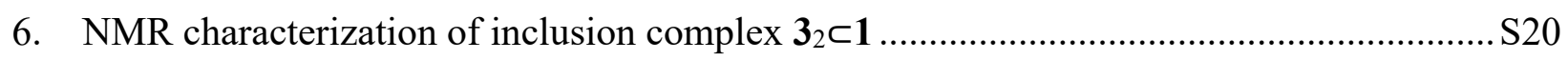

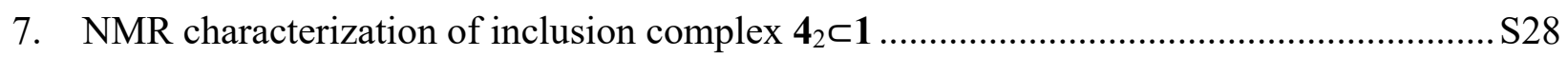

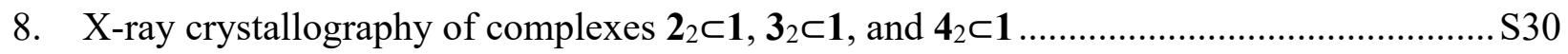

9. Steady-state optical properties of $\mathbf{2}-\mathbf{4}$ and their inclusion complexes ................................S35

10. Time-resolved optical properties of 2-4 and their inclusion complexes .......................... S40

11. Competitive binding of BODIPY 2 vs azobenzene guests ............................................ S42

12. Reversible switching between $\mathrm{H}$ - and J-aggregates of BODIPY 4 ….............................. 443

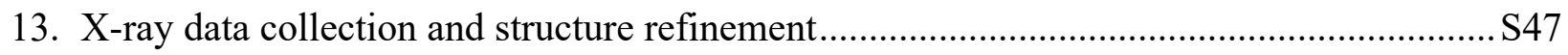

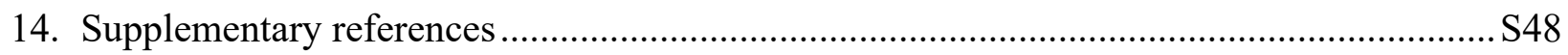




\section{Materials and methods}

All commercial chemicals were used as received unless stated otherwise. Solvents were dried according to standard procedures. Synthesis was carried out in oven-dried glassware. NMR spectra were recorded on a Bruker Avance III $400 \mathrm{MHz}$ spectrometer, a Bruker Avance III HD $500 \mathrm{MHz}$ spectrometer, or a Bruker Avance III $800 \mathrm{MHz}$ spectrometer. Chemical shifts $(\delta)$ are given in ppm relative to residual proton solvent resonances (4.79 ppm for $\mathrm{D}_{2} \mathrm{O}$ and $7.26 \mathrm{ppm}$ for $\mathrm{CDCl}_{3}$ ). ${ }^{1} \mathrm{H}$ DOSY measurements were performed on a Bruker Avance III $500 \mathrm{MHz}$ spectrometer with temperature and gradient calibration prior to the measurements (the diffusion coefficient of the solvent was used as a calibration standard). A constant temperature of $298 \mathrm{~K}$ was maintained during all NMR measurements unless stated otherwise. Solution and solid-state UV-vis absorption spectra were recorded with a Shimadzu UV-2700 or a UV-3600 spectrophotometer. Emission and excitation spectra were recorded with a Shimadzu spectrofluorophotometer RF-5301 PC. Fluorescence quantum yields were determined on a Quantaurus-QY Absolute PL quantum yield spectrometer. Experimental details about the time-resolved fluorescence and absorbance spectroscopy can be found in Section 10. For details on the X-ray data collection and refinement, see Section 13. 


\section{Synthesis of cage 1}

Coordination cage $\mathbf{1}$ was synthesized based on a reported literature procedure. ${ }^{1}$

${ }^{1} \mathrm{H}$ NMR $\left(500 \mathrm{MHz}, \mathrm{D}_{2} \mathrm{O}\right): \delta=9.12\left(\mathrm{~s}, 8 \mathrm{H}, \mathbf{1}_{4}\right), 8.83\left(\mathrm{~s}, 4 \mathrm{H}, \mathbf{1}_{1}\right), 7.75\left(\mathrm{~s}, 4 \mathrm{H}, \mathbf{1}_{\mathbf{8}}\right), 7.73\left(\mathrm{~s}, 4 \mathrm{H}, \mathbf{1}_{3}\right), 7.71(\mathrm{~s}, 8 \mathrm{H}$, 17), $7.66\left(\mathrm{~s}, 8 \mathrm{H}, \mathbf{1}_{6}\right), 7.56\left(\mathrm{~s}, 12 \mathrm{H}, \mathbf{1}_{2+5}\right), 3.13\left(\mathrm{~s}, 24 \mathrm{H}, \mathbf{1}_{11}\right), 2.84-2.66\left(\mathrm{~m}, 72 \mathrm{H}, \mathbf{1}_{12}\right)$.

${ }^{13} \mathrm{C}$ NMR $\left(100 \mathrm{MHz}, \mathrm{D}_{2} \mathrm{O}\right): \delta=138.04(\mathbf{1}), 137.76\left(\mathbf{1}_{\mathbf{1 0}}\right), 137.51\left(\mathbf{1}_{\mathbf{4}}\right), 137.26\left(\mathbf{1}_{\mathbf{1}}\right), 128.73\left(\mathbf{1}_{\mathbf{2}+5}\right), 120.74\left(\mathbf{1}_{\mathbf{6}}\right)$, $120.51\left(\mathbf{1}_{3}\right), 114.78\left(\mathbf{1}_{\mathbf{8}}\right), 113.09\left(\mathbf{1}_{7}\right), 62.52\left(\mathbf{1}_{11}\right), 50.29\left(\mathbf{1}_{12}\right), 50.24\left(\mathbf{1}_{12}\right), 50.02\left(\mathbf{1}_{12}\right)$.

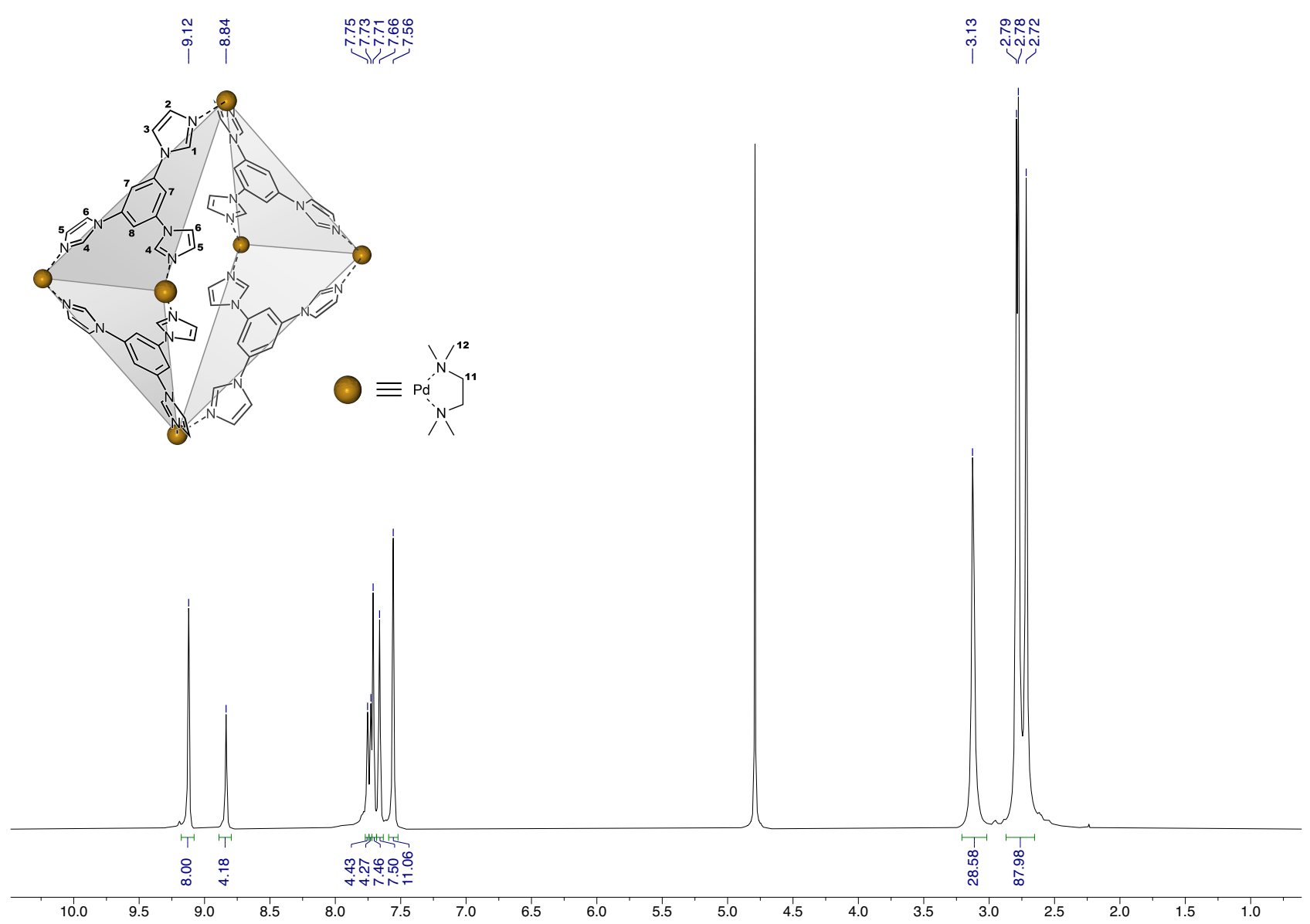

Figure S1. ${ }^{1} \mathrm{H}$ NMR spectrum of 1 (500 MHz, $\left.\mathrm{D}_{2} \mathrm{O}, 298 \mathrm{~K}\right)$. 


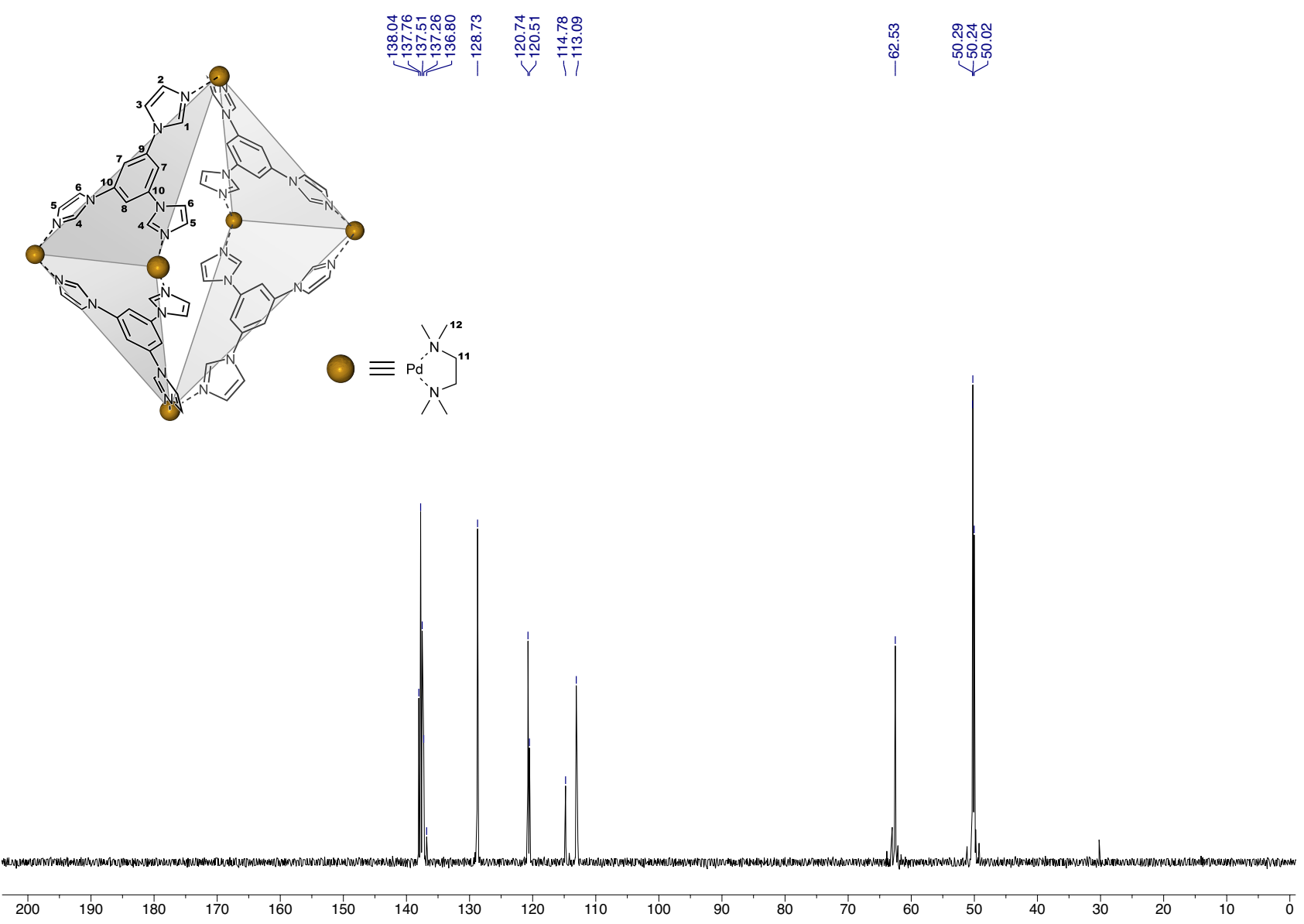

Figure S2. ${ }^{13} \mathrm{C}$ NMR spectrum of $\mathbf{1}\left(100 \mathrm{MHz}, \mathrm{D}_{2} \mathrm{O}, 298 \mathrm{~K}\right)$. 
3. Synthesis and characterization of BODIPY dyes $\mathbf{2 , 3}$, and $\mathbf{4}$

BODIPY 2 was synthesized based on a reported literature procedure. ${ }^{2}$

${ }^{1} \mathrm{H}$ NMR (400 MHz, CDCl $)$ ): $\delta=6.05$ (s, 2H, 24), 2.57 (s, 3H, 23), 2.52 (s, 6H, 21), 2.41 (s, 6H, 2 2 ).

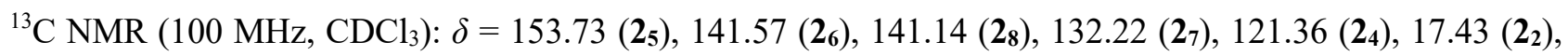
$16.49\left(\mathbf{2}_{3}\right), 14.55\left(\mathbf{2}_{1}\right)$.

${ }^{19} \mathrm{~F}$ NMR (376 MHz, $\left.\mathrm{CDCl}_{3}\right): \delta=-147.42$ (q).
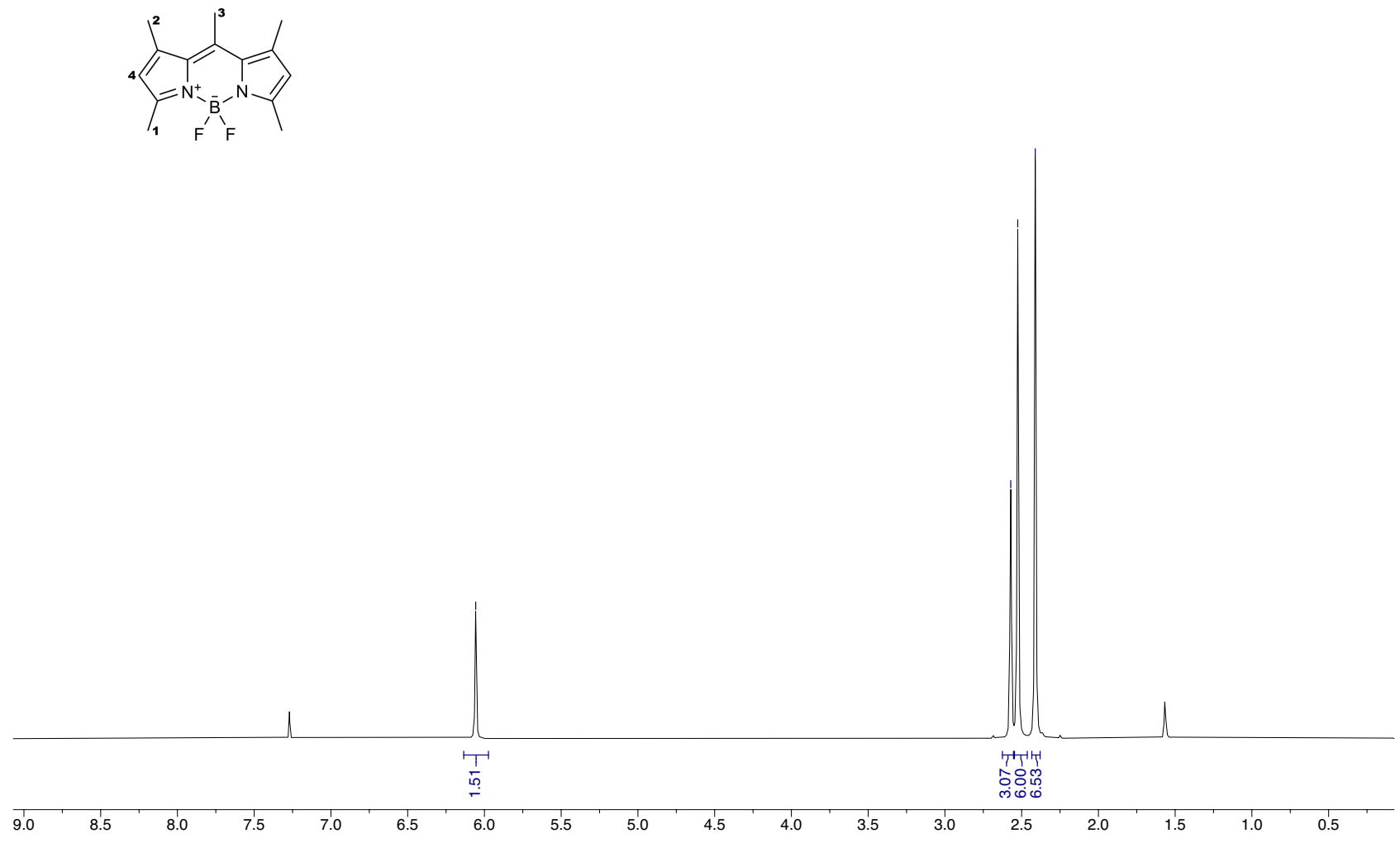

Figure S3. ${ }^{1} \mathrm{H}$ NMR spectrum of $2\left(400 \mathrm{MHz}, \mathrm{CDCl}_{3}, 298 \mathrm{~K}\right)$. 


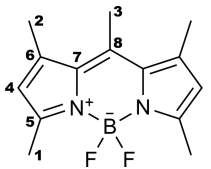

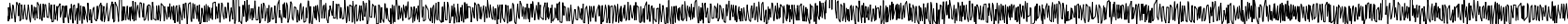

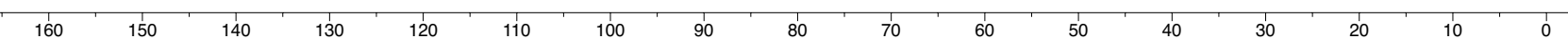

Figure S4. ${ }^{13} \mathrm{C}$ NMR spectrum of $2\left(100 \mathrm{MHz}, \mathrm{CDCl}_{3}, 298 \mathrm{~K}\right)$.

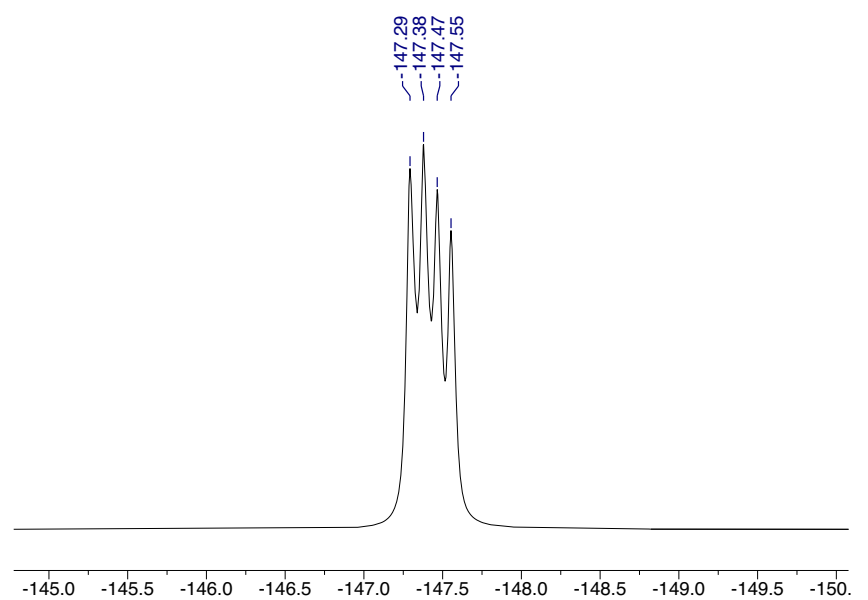

Figure S5. ${ }^{19} \mathrm{~F}$ NMR spectrum of $2\left(376 \mathrm{MHz}, \mathrm{CDCl}_{3}, 298 \mathrm{~K}\right)$. 
BODIPY 3 was obtained as a byproduct in the synthesis of 8-acetoxymethyl-1,2,3,5,6,7-hexamethyl pyrromethene fluoroborate. Specifically, acetoxyacetyl chloride $(0.87 \mathrm{~mL} ; 8.1 \mathrm{mmol} ; 1.2 \mathrm{eq})$ was slowly added to a solution of 2,3,4-trimethyl- $1 \mathrm{H}$-pyrrole $(1.478 \mathrm{~g} ; 13.5 \mathrm{mmol} ; 2.0 \mathrm{eq})$ in dry $\mathrm{CH}_{2} \mathrm{Cl}_{2}(6 \mathrm{~mL})$. During the addition, the solution warmed up and turned deep-red. Then, the mixture was refluxed for $1 \mathrm{~h}$, cooled down, and poured onto $n$-hexane $(100 \mathrm{~mL})$. The solvent was evaporated to afford a deep-red solid. The solid was redissolved in dry $\mathrm{CH}_{2} \mathrm{Cl}_{2}(70 \mathrm{~mL})$ containing $6.8 \mathrm{~mL}(39 \mathrm{mmol})$ of diisopropylethylamine. After having been stirred for $10 \mathrm{~min}$ at room temperature, $\mathrm{BF}_{3} \cdot \mathrm{OEt}_{2}(7.2 \mathrm{~mL} ; 58 \mathrm{mmol})$ was added dropwise and the solution was stirred for $1 \mathrm{~h}$ at the same temperature, turning violet. Then, the reaction was discontinued and the mixture was washed with a saturated aqueous solution of $\mathrm{NaHCO}_{3}(3 \times 100 \mathrm{~mL})$ and dried over $\mathrm{Na}_{2} \mathrm{SO}_{4}$. The solvent was evaporated and the resulting solid was purified by column chromatography $\left(\mathrm{CH}_{2} \mathrm{Cl}_{2} /\right.$ petroleum ether 1/1). BODIPY 3 was isolated as a dark-purple solid. Yield: $167 \mathrm{mg}(0.55 \mathrm{mmol}, 8 \%)$.

The peaks in the NMR spectra were assigned by analyzing 2D NMR spectra (not shown).

${ }^{1} \mathrm{H}$ NMR (400 MHz, CDCl $): \delta=10.60$ (s, 1H, 33), 2.49 (s, 6H, 3 $\mathbf{3}_{1}$ ), 2.02 (s, 6H, 32), 1.91 (s, 6H, 34).

${ }^{13} \mathrm{C} \mathrm{NMR}\left(100 \mathrm{MHz}, \mathrm{CDCl}_{3}\right): \delta=194.19\left(\mathbf{3}_{3}\right), 157.12\left(\mathbf{3}_{5}\right), 137.07\left(\mathbf{3}_{\mathbf{6}}\right), 134.87\left(\mathbf{3}_{\mathbf{8}}\right), 128.06\left(\mathbf{3}_{7}\right), 127.34\left(\mathbf{2}_{\mathbf{9}}\right)$, $13.22\left(\mathbf{3}_{1}\right), 13.18\left(\mathbf{3}_{2}\right), 9.11\left(\mathbf{3}_{4}\right)$.

${ }^{19} \mathrm{~F}$ NMR (376 MHz, $\left.\mathrm{CDCl}_{3}\right): \delta=-146.57$ (q).
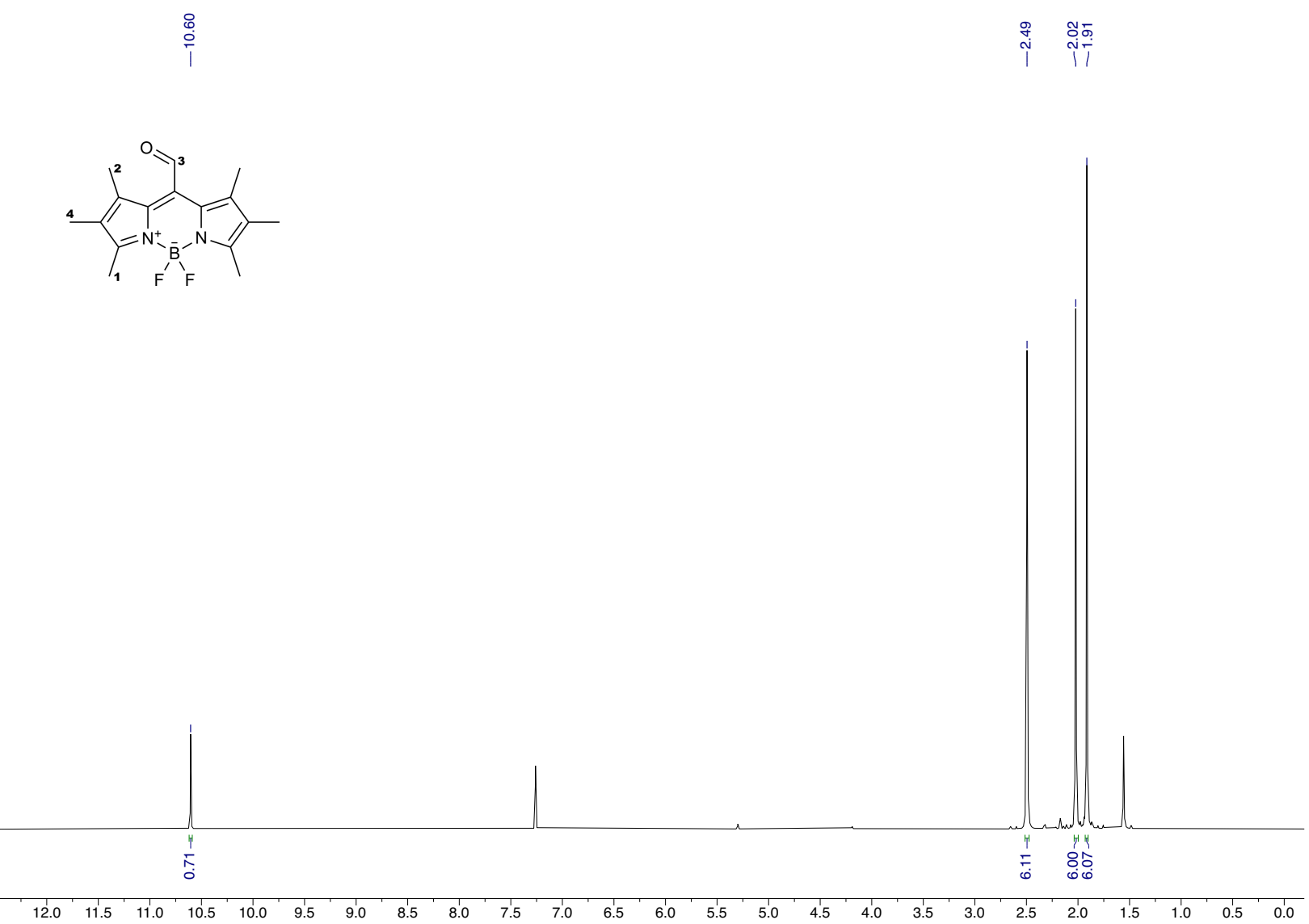

Figure S6. ${ }^{1} \mathrm{H}$ NMR spectrum of 3 (400 MHz, $\left.\mathrm{CDCl}_{3}, 298 \mathrm{~K}\right)$. 

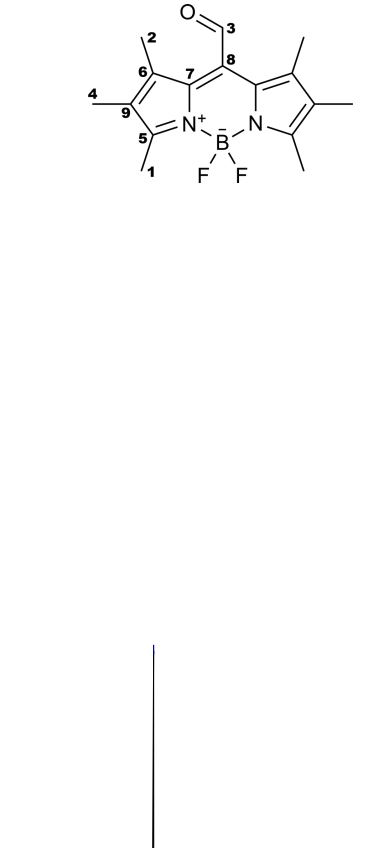

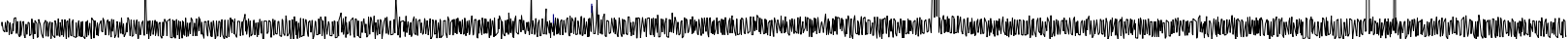

$\begin{array}{llllllllllllllllllllllllllll}210 & 200 & 190 & 180 & 170 & 160 & 150 & 140 & 130 & 120 & 110 & 100 & 90 & 80 & 70 & 60 & 50 & 40 & 30 & 20 & 10 & 0 & -10\end{array}$

Figure S7. ${ }^{13} \mathrm{C}$ NMR spectrum of $3\left(100 \mathrm{MHz}, \mathrm{CDCl}_{3}, 298 \mathrm{~K}\right)$.

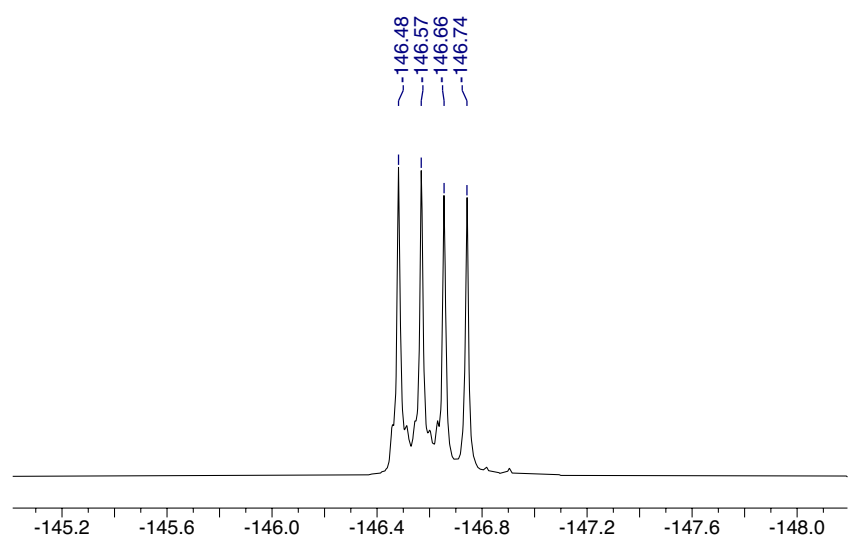

Figure S8. ${ }^{19} \mathrm{~F}$ NMR spectrum of $3\left(376 \mathrm{MHz}, \mathrm{CDCl}_{3}, 298 \mathrm{~K}\right)$. 


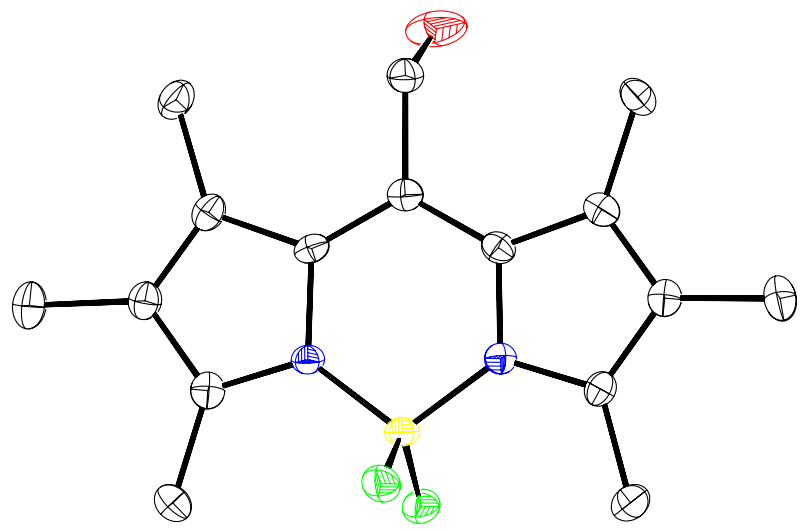

Figure S9. ORTEP representation of the X-ray structure of BODIPY 3 (thermal ellipsoids at a $50 \%$ probability level). C, gray; N, blue; O, red; B, yellow; F, green (note that the X-ray structures of BODIPYs $\mathbf{2}$ and $\mathbf{4}$ have been reported previously ${ }^{3,4}$ ).

BODIPY 4 was synthesized based on a reported literature procedure. ${ }^{4}$

${ }^{1} \mathrm{H} \mathrm{NMR}\left(400 \mathrm{MHz}, \mathrm{CDCl}_{3}\right): \delta=6.15$ (s, 2H, 43), 2.54 (s, 6H, 4) $)$ 2.33-2.25 (m, 6H, 42).

${ }^{13} \mathrm{C}$ NMR (100 MHz, $\left.\mathrm{CDCl}_{3}\right): \delta=158.84\left(\mathbf{4}_{5}\right), 143.14\left(\mathbf{4}_{6}\right), 131.3\left(\mathbf{4}_{7}\right), 124.41\left(\mathbf{4}_{\mathbf{8}}\right), 123.71\left(\mathbf{4}_{4}\right), 120.98\left(\mathbf{4}_{3}\right)$, $15.87\left(\mathbf{4}_{2}\right), 15.00\left(\mathbf{4}_{\mathbf{1}}\right)$.

${ }^{19} \mathrm{~F}$ NMR (376 MHz, $\left.\mathrm{CDCl}_{3}\right): \delta=-53.51\left(\mathrm{t}, 3 \mathrm{~F}, \mathbf{4}_{\mathrm{CF} 3}\right),-146.86\left(\mathrm{q}, 2 \mathrm{~F}, \mathbf{4}_{\mathrm{BF} 2}\right)$.

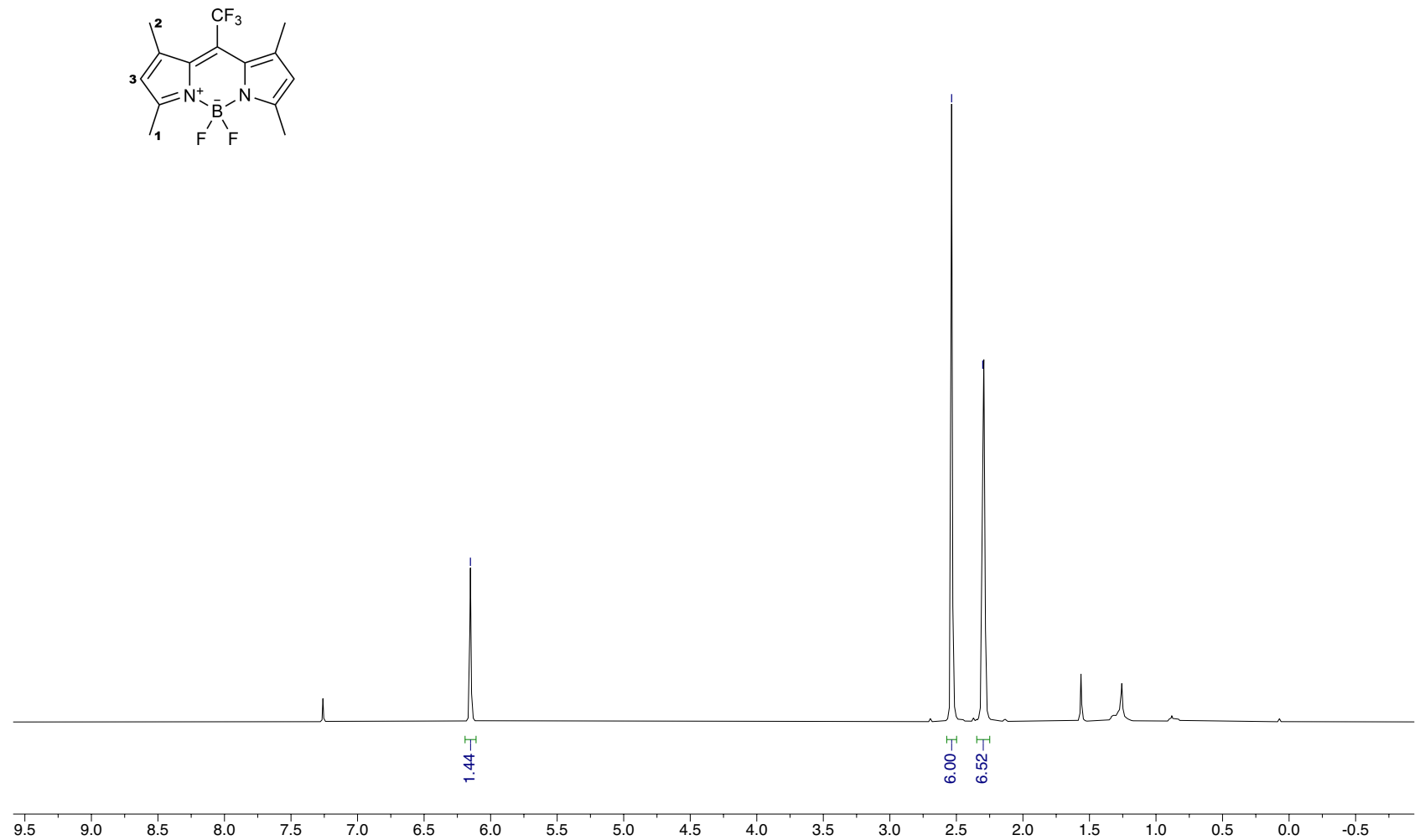

Figure S10. ${ }^{1} \mathrm{H}$ NMR spectrum of 4 (400 MHz, $\left.\mathrm{CDCl}_{3}, 298 \mathrm{~K}\right)$. 

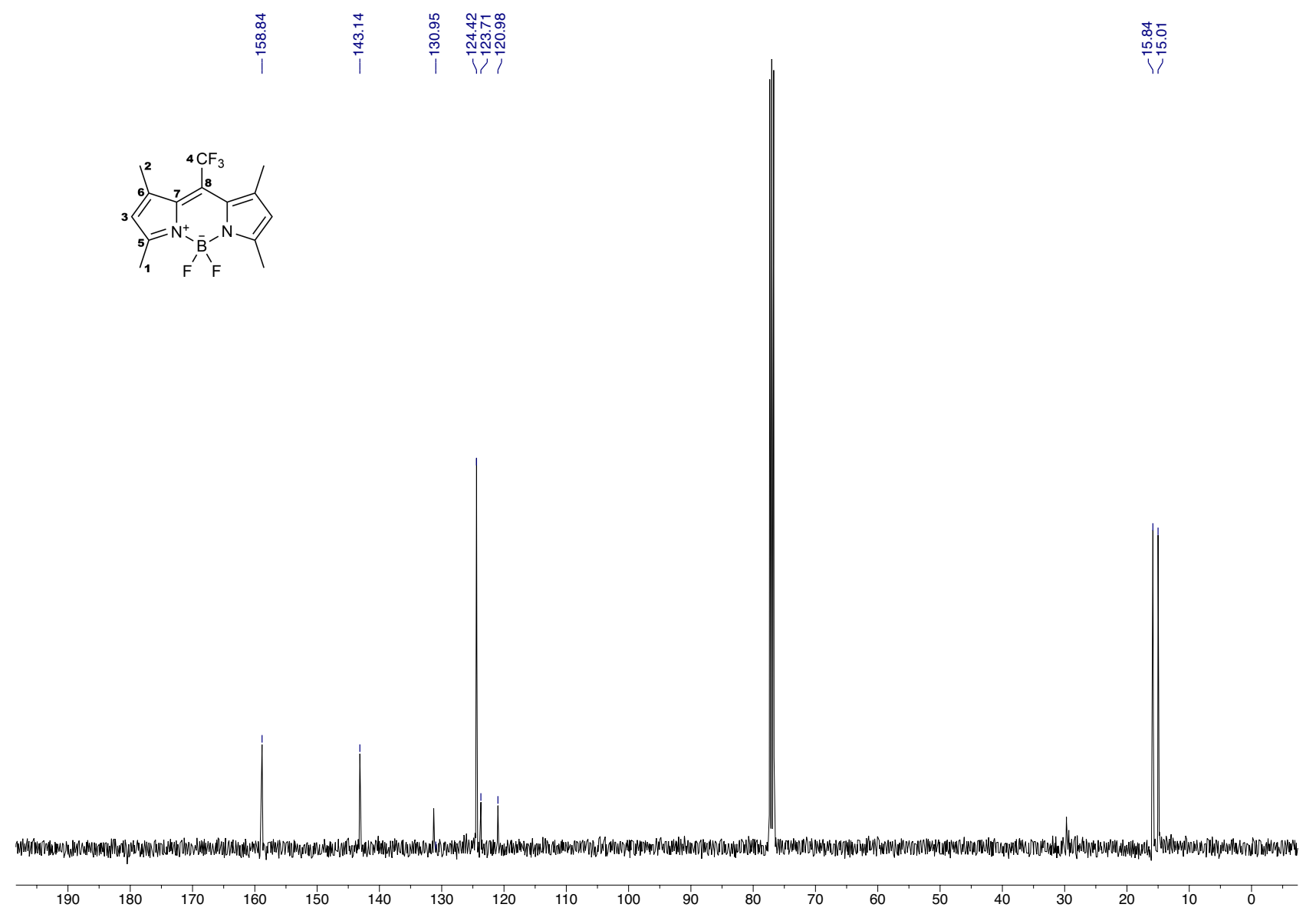

Figure S11. ${ }^{13} \mathrm{C}$ NMR spectrum of $4\left(100 \mathrm{MHz}, \mathrm{CDCl}_{3}, 298 \mathrm{~K}\right)$.

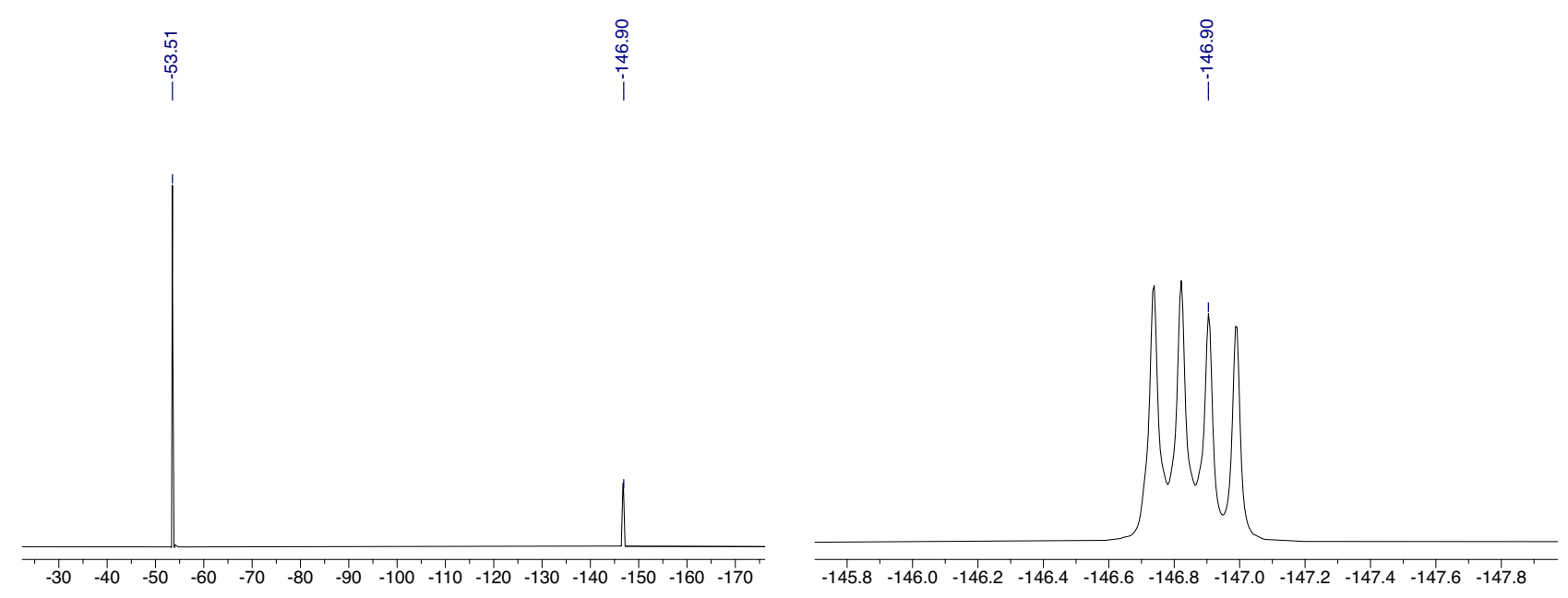

Figure S12. Full-range (left) and partial (right) ${ }^{19} \mathrm{~F}$ NMR spectrum of 4 ( $\left.376 \mathrm{MHz}, \mathrm{CDCl}_{3}, 298 \mathrm{~K}\right)$. 


\section{Encapsulation of BODIPYs $\mathbf{2}, \mathbf{3}$, and $\mathbf{4}$ within cage $\mathbf{1}$}

General procedure for encapsulation: Cage $1(9.0 \mathrm{mg}, 2.8 \mu \mathrm{mol})$ was dissolved in $\mathrm{D}_{2} \mathrm{O}(0.7 \mathrm{~mL})$. The resulting colorless solution was added to an excess ( $>5$ eq) of solid $\mathbf{2 , 3}$, or $\mathbf{4}$ (none of which is soluble in water) and the resulting suspensions were stirred overnight in the dark at ambient temperature. Then, the undissolved solids were removed by several cycles of centrifugation, resulting in clear, intensely colored solutions. Stirring for a longer time, heating, and applying sonication did not increase the encapsulation yields. The resulting solutions were stable in the dark at ambient temperature for at least several months (verified by NMR and UV-vis absorption spectroscopy).

The intense colorization of the aqueous solutions of $\mathbf{1}$ in the visible area allowed us to follow the uptake of each BODIPY dye by the cage over time. To this end, aliquots from the suspensions were taken at various times, subjected to repeated centrifugations, and analyzed by UV-vis absorption spectroscopy. Representative UV-vis spectra are shown in Figure S13. Based on the results replotted in Figure S14, we concluded that the uptake of all the BODIPY dyes is complete within $\sim 10 \mathrm{~h}$, with minor differences between dyes $\mathbf{2 , 3}$, and $\mathbf{4}$.
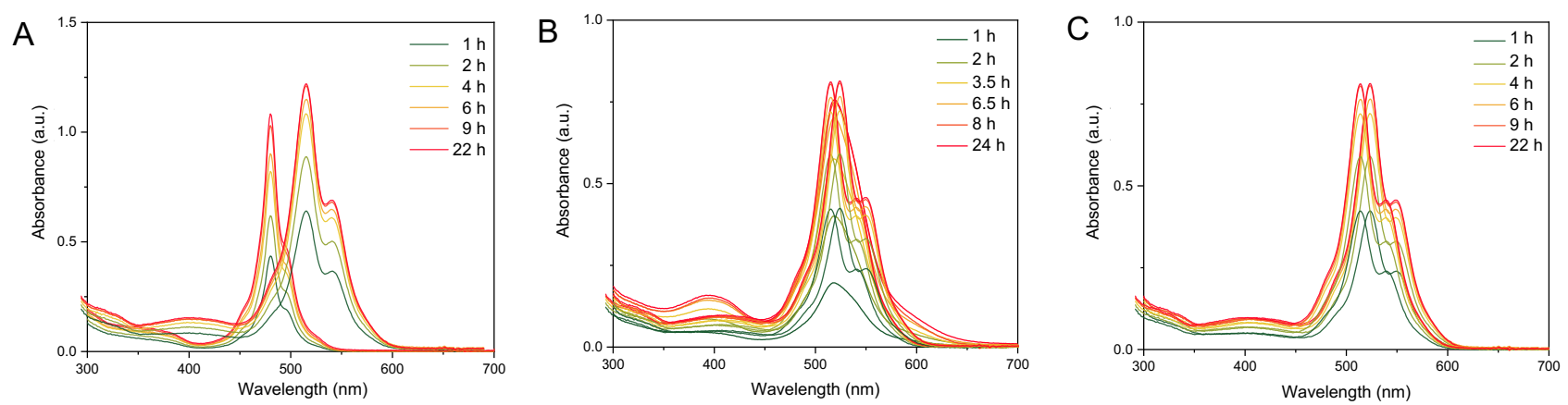

Figure S13. Uptake of 2 (A), 3 (B), and 4 (C) by cage 1 over time followed by UV-vis absorption spectroscopy.
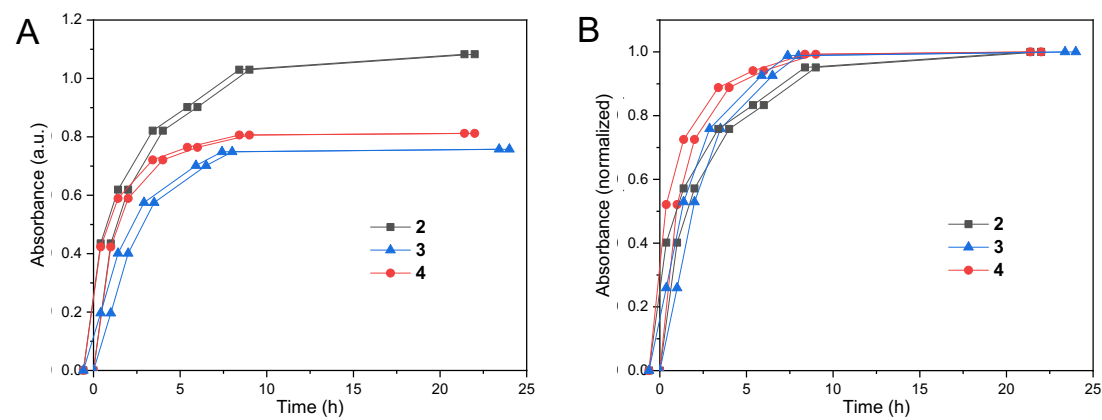

Figure S14. Absolute (A) and normalized (B) profiles of the uptake of BODIPYs 2, 3, and $\mathbf{4}$ by cage $\mathbf{1}$. Absorbance was followed at $480 \mathrm{~nm}, 519 \mathrm{~nm}$, and $523 \mathrm{~nm}$ for 2, 3, and 4, respectively. 


\section{NMR characterization of inclusion complex $\mathbf{2}_{2} \subset 1$}

Inclusion complex $\mathbf{2}_{2} \subset \mathbf{1}$ was obtained in $\sim 50 \%$ yield as determined by ${ }^{1} \mathrm{H}$ NMR spectroscopy. To help assign peaks due to $\mathbf{1}$ vs $\mathbf{2}$, as-prepared $\mathbf{2}_{2} \subset \mathbf{1}$ was treated with extra free $\mathbf{1}$ (see Figure S16).

${ }^{1} \mathrm{H}$ NMR (500 MHz, $\left.\mathrm{D}_{2} \mathrm{O}\right): \delta=9.55\left(\mathrm{~s}, 4 \mathrm{H}, \mathbf{1}_{\mathbf{4}}\right), 9.29$ (s, 4H, 14) $), 8.81$ (s, 4H, $\left.\mathbf{1}_{\mathbf{1}+\mathbf{1}}\right), 8.31\left(\mathrm{~s}, 2 \mathrm{H}, \mathbf{1}_{\mathbf{8}}\right), 8.07$ (s,

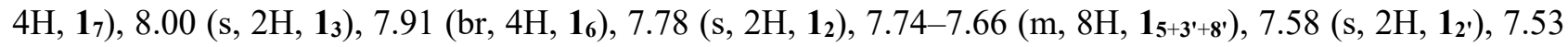

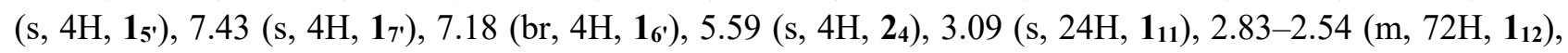
1.87 (br, 12H, 2 1 ), $0.62\left(\mathrm{~s}, 12 \mathrm{H}, \mathbf{2}_{2}\right), 0.26\left(\mathrm{~s}, 6 \mathrm{H}, \mathbf{2}_{3}\right)$.

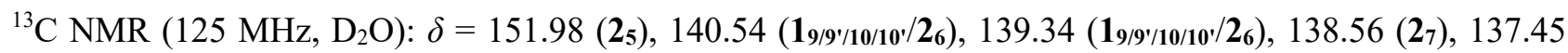

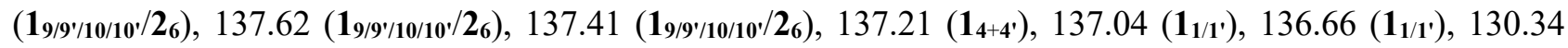

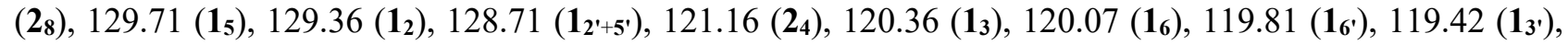
$111.43\left(\mathbf{1}_{\mathbf{8}}\right), 110.68\left(\mathbf{1}_{7}\right), 110.00\left(\mathbf{1}_{\mathbf{8}^{\prime}}\right), 109.24\left(\mathbf{1}_{7^{\prime}}\right), 62.61\left(\mathbf{1}_{\mathbf{1 2}}\right), 50.43-49.91\left(\mathbf{1}_{11}\right), 14.92\left(\mathbf{2}_{\mathbf{2}}\right), 13.53\left(\mathbf{2}_{\mathbf{3}}\right), 13.31$ (21).

${ }^{19} \mathrm{~F}$ NMR (470 MHz, $\left.\mathrm{D}_{2} \mathrm{O}\right): \delta=(-142.72)-(-143.17)\left(\mathrm{m}, 2 \mathrm{~F}, \mathbf{2}_{\mathrm{BF} 2}\right),-144.70\left(\mathrm{br}, 2 \mathrm{~F}, \mathbf{2}_{\mathrm{BF} 2}\right)$.

${ }^{1} \mathrm{H}$ DOSY NMR $\left(500 \mathrm{MHz}, \mathrm{D}_{2} \mathrm{O}\right): D=0.15 \times 10^{-5} \mathrm{~cm}^{2} / \mathrm{s}$.
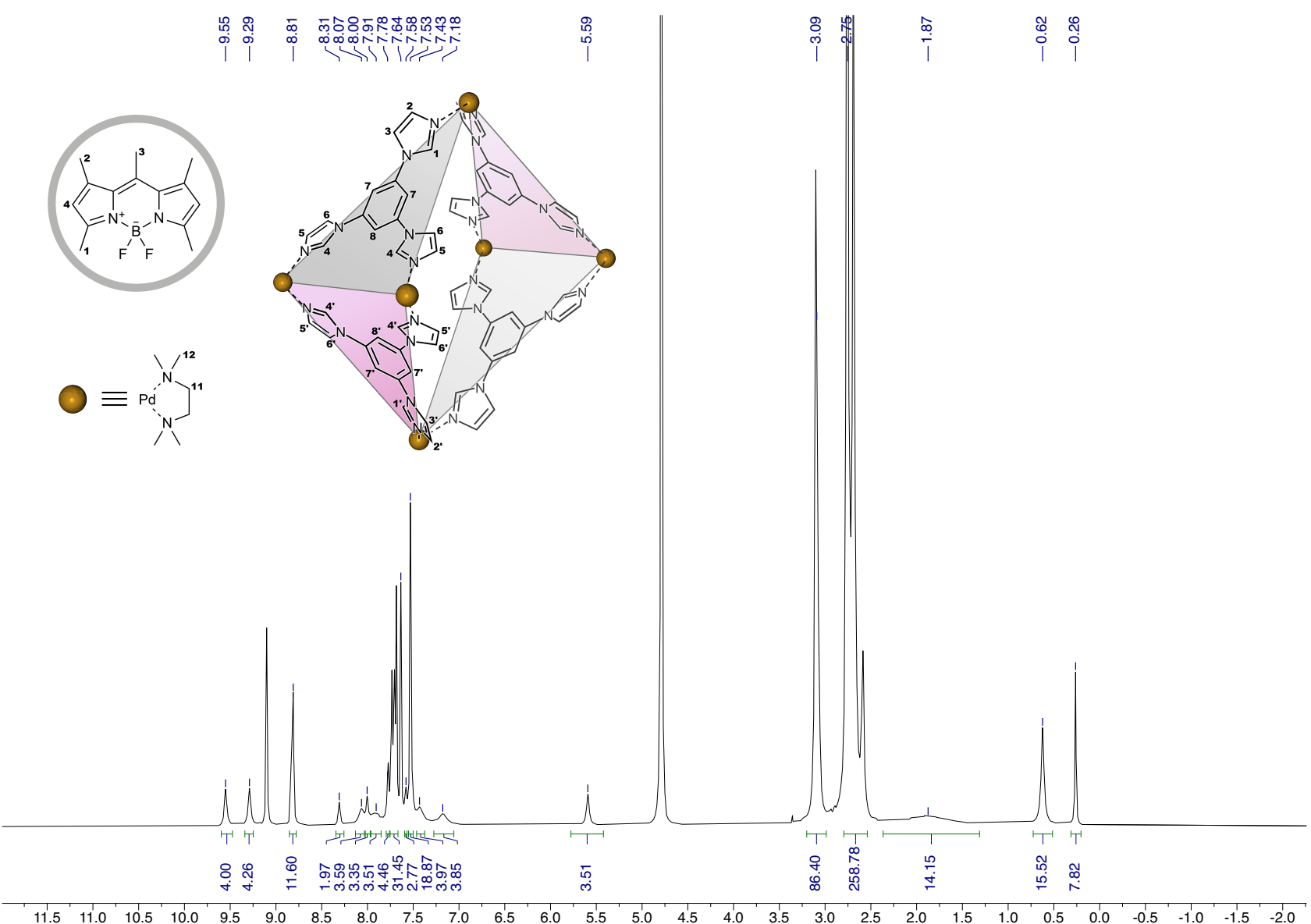

Figure S15. ${ }^{1} \mathrm{H}$ NMR spectrum of $\mathbf{2}_{2} \subset \mathbf{1}\left(500 \mathrm{MHz}, \mathrm{D}_{2} \mathrm{O}, 298 \mathrm{~K}\right)$. 

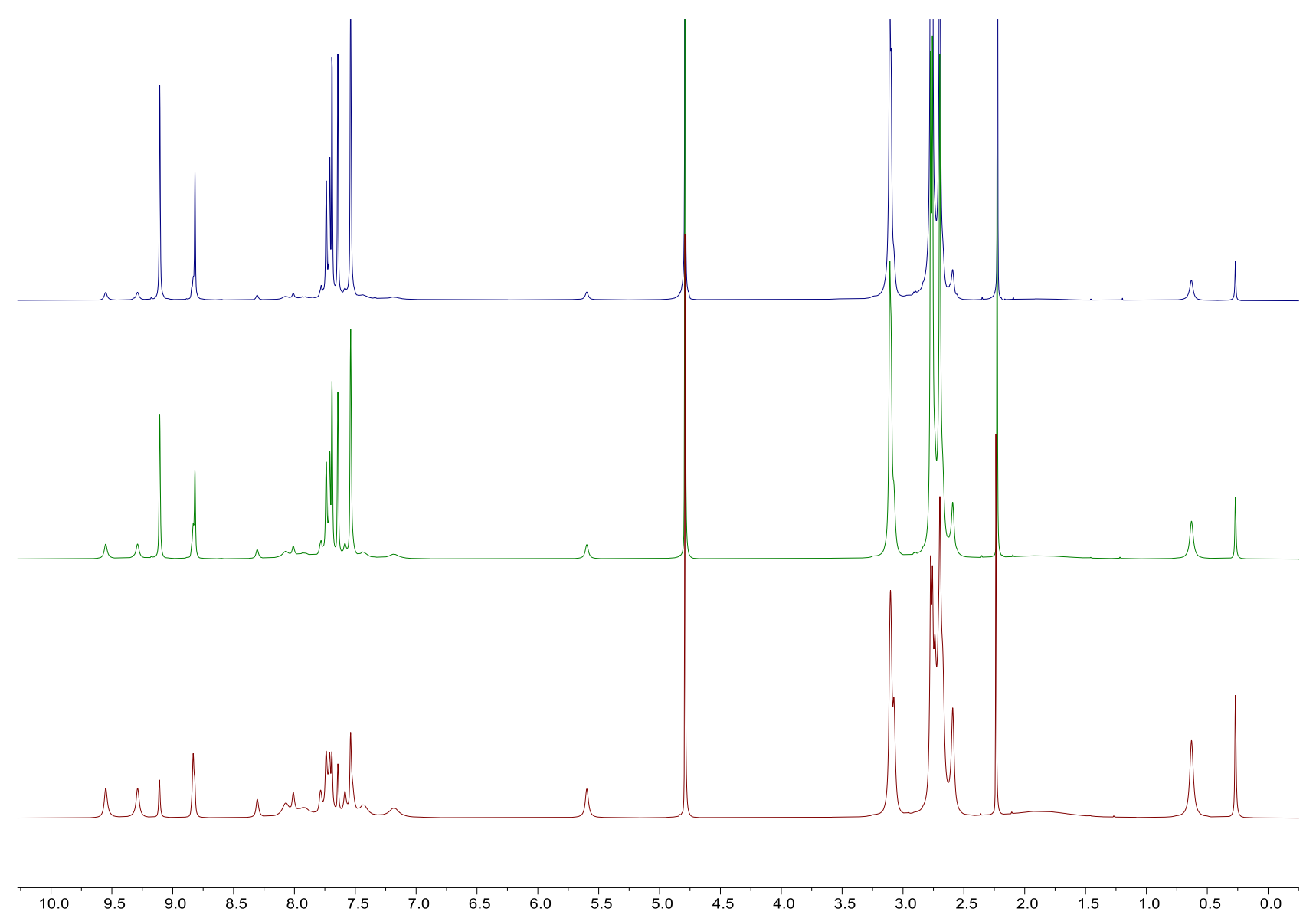

Figure S16. ${ }^{1} \mathrm{H}$ NMR spectrum of as-prepared $\mathbf{2}_{2} \subset \mathbf{1}$ (in the presence of free cage 1, which always remains partially unfilled) (bottom; red), after the addition of an extra 1 equiv of free 1 (center; green), and after the addition of an extra 2 equiv of free 1 (i.e., a total of 3 equiv of 1) (top; dark-blue) (400 MHz, $\mathrm{D}_{2} \mathrm{O}, 298 \mathrm{~K}$ ). 

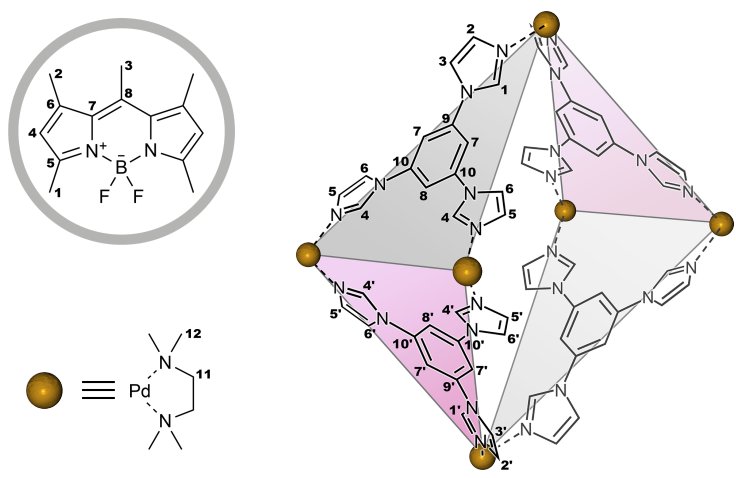

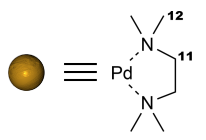

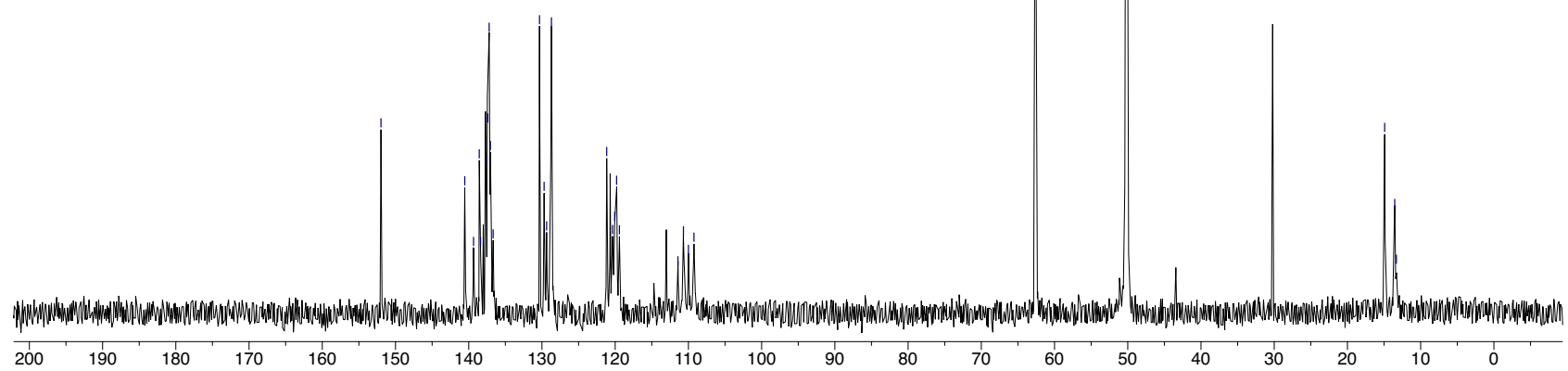

Figure S17. ${ }^{13} \mathrm{C}$ NMR spectrum of $\mathbf{2}_{2} \subset \mathbf{1}\left(125 \mathrm{MHz}, \mathrm{D}_{2} \mathrm{O}, 298 \mathrm{~K}\right)$.

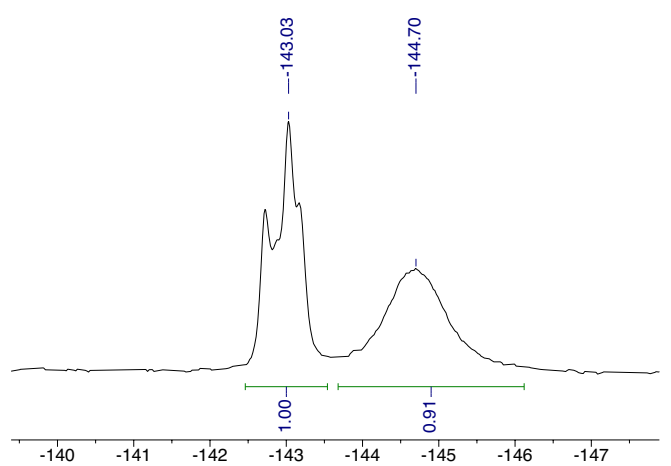

Figure S18. ${ }^{19} \mathrm{~F}$ NMR spectrum of $\boldsymbol{2}_{2} \subset \mathbf{1}\left(470 \mathrm{MHz}, \mathrm{D}_{2} \mathrm{O}, 298 \mathrm{~K}\right)$. 


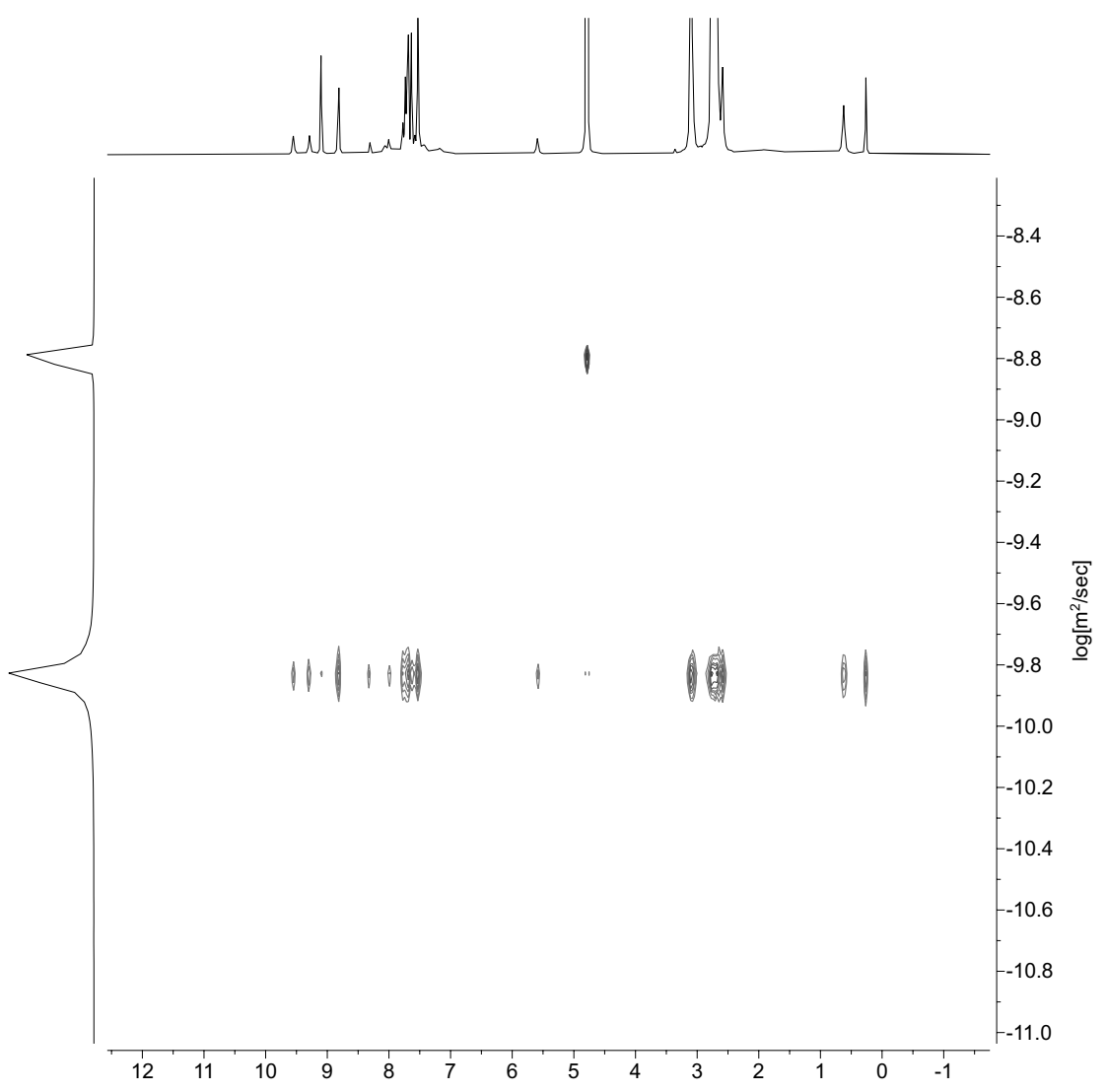

Figure S19. ${ }^{1} \mathrm{H}$ DOSY NMR spectrum of $\mathbf{2}_{2} \subset \mathbf{1}\left(500 \mathrm{MHz}, \mathrm{D}_{2} \mathrm{O}, 298 \mathrm{~K}\right)$.

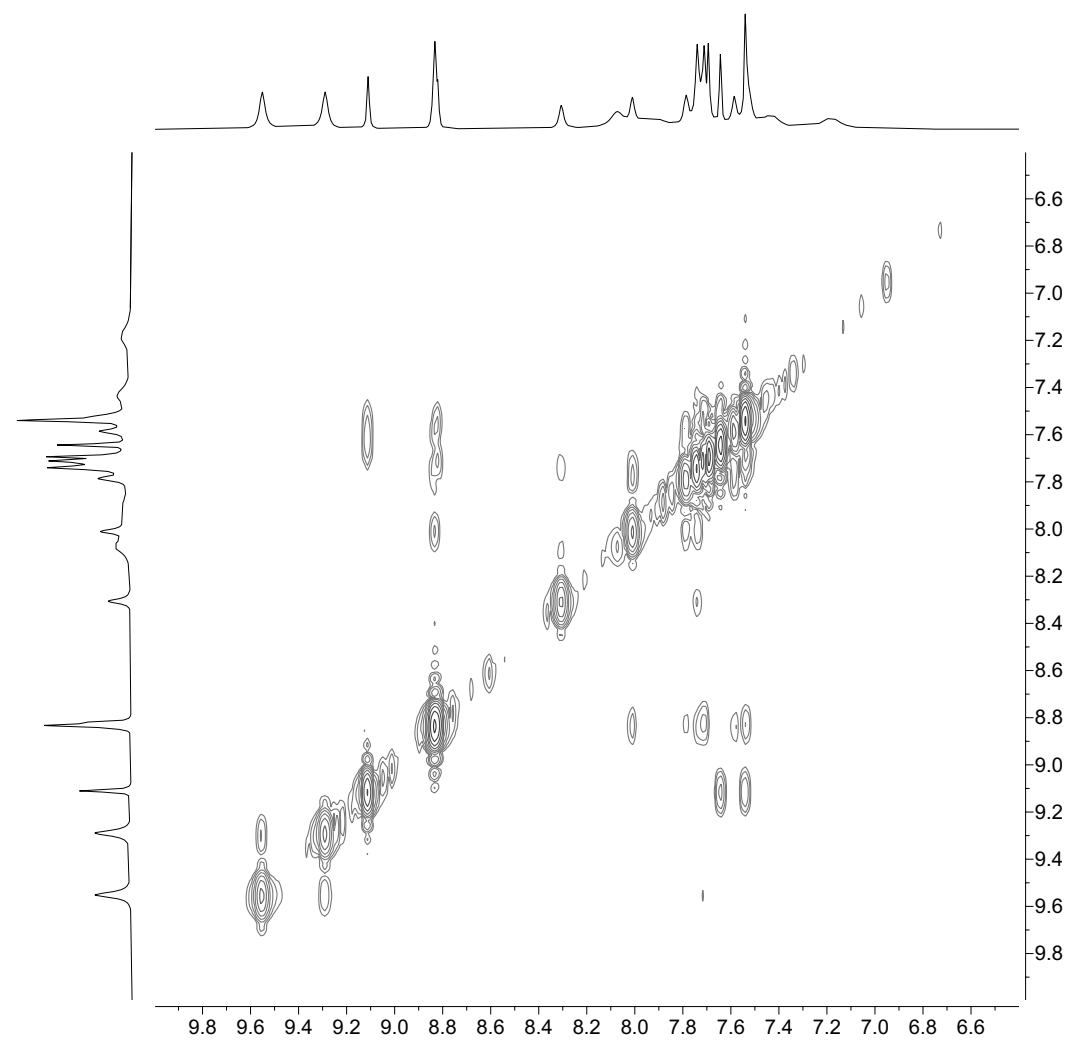

Figure S20. Partial ${ }^{1} \mathrm{H}-{ }^{1} \mathrm{H}$ COSY NMR spectrum of $\mathbf{2}_{2} \subset \mathbf{1}\left(500 \mathrm{MHz}, \mathrm{D}_{2} \mathrm{O}, 298 \mathrm{~K}\right)$. 


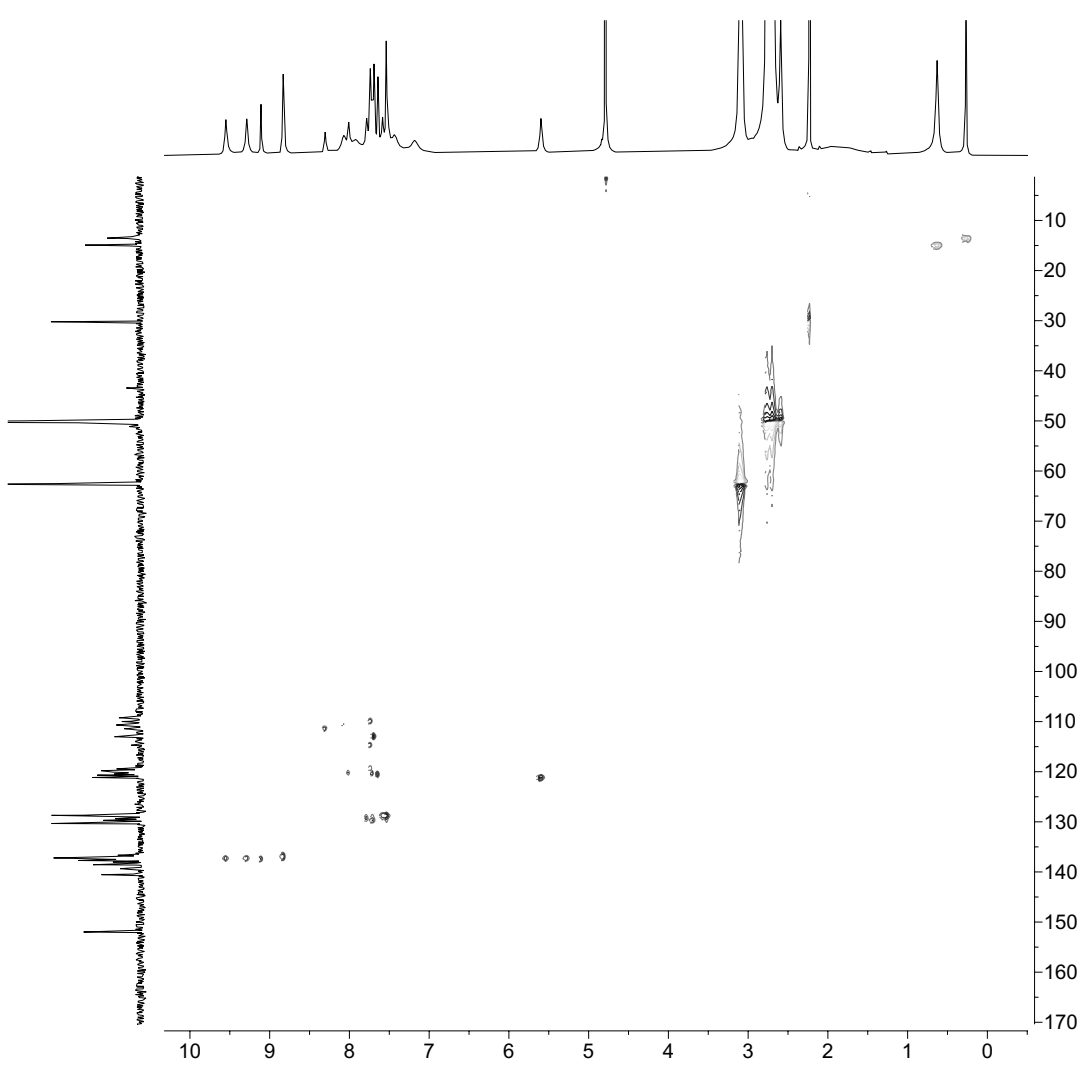

Figure S21. ${ }^{1} \mathrm{H}-{ }^{13} \mathrm{C}$ HSQC NMR spectrum of $\mathbf{2}_{2} \subset \mathbf{1}\left(500 \mathrm{MHz}, \mathrm{D}_{2} \mathrm{O}, 298 \mathrm{~K}\right)$.

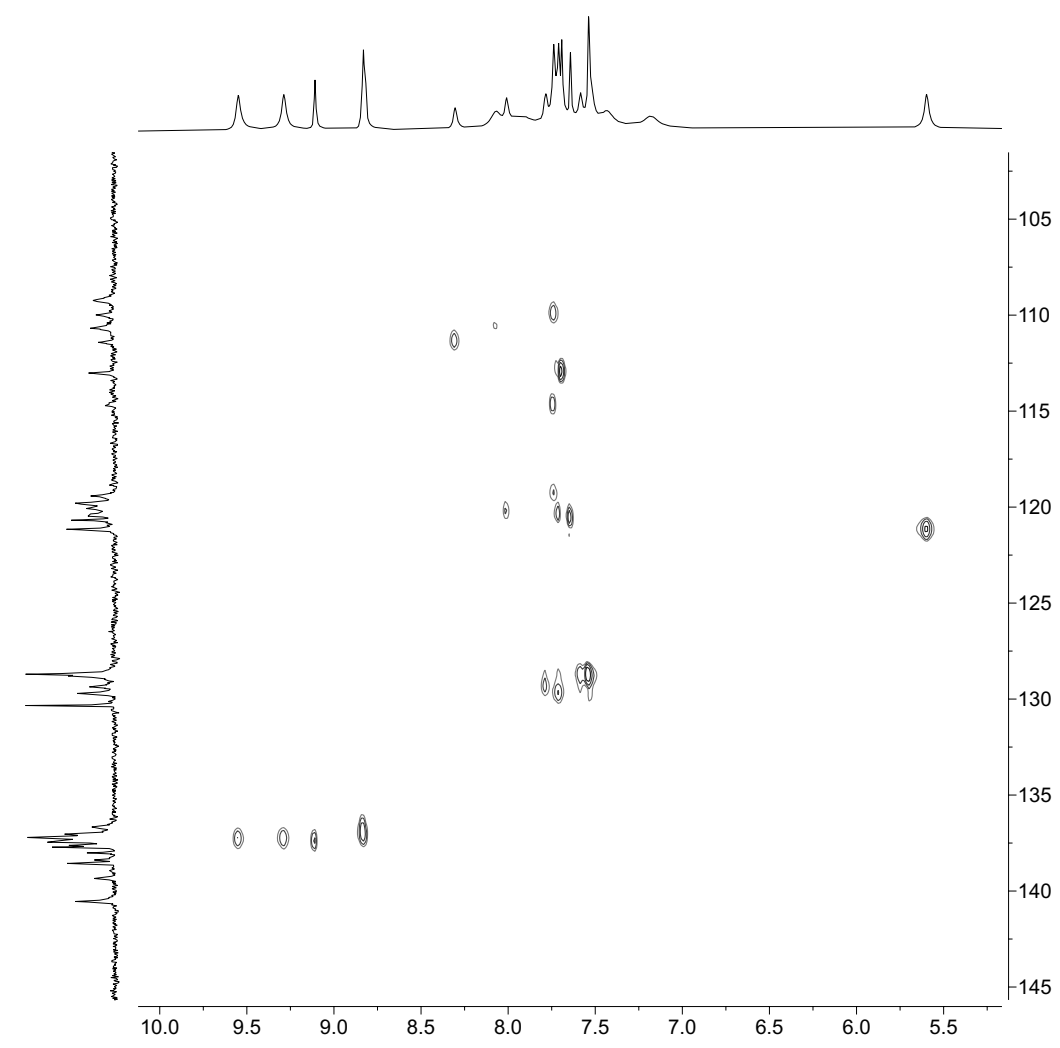

Figure S22. Partial ${ }^{1} \mathrm{H}-{ }^{13} \mathrm{C}$ HSQC NMR spectrum of $\mathbf{2}_{2} \subset \mathbf{1}\left(500 \mathrm{MHz}, \mathrm{D}_{2} \mathrm{O}, 298 \mathrm{~K}\right)$. 


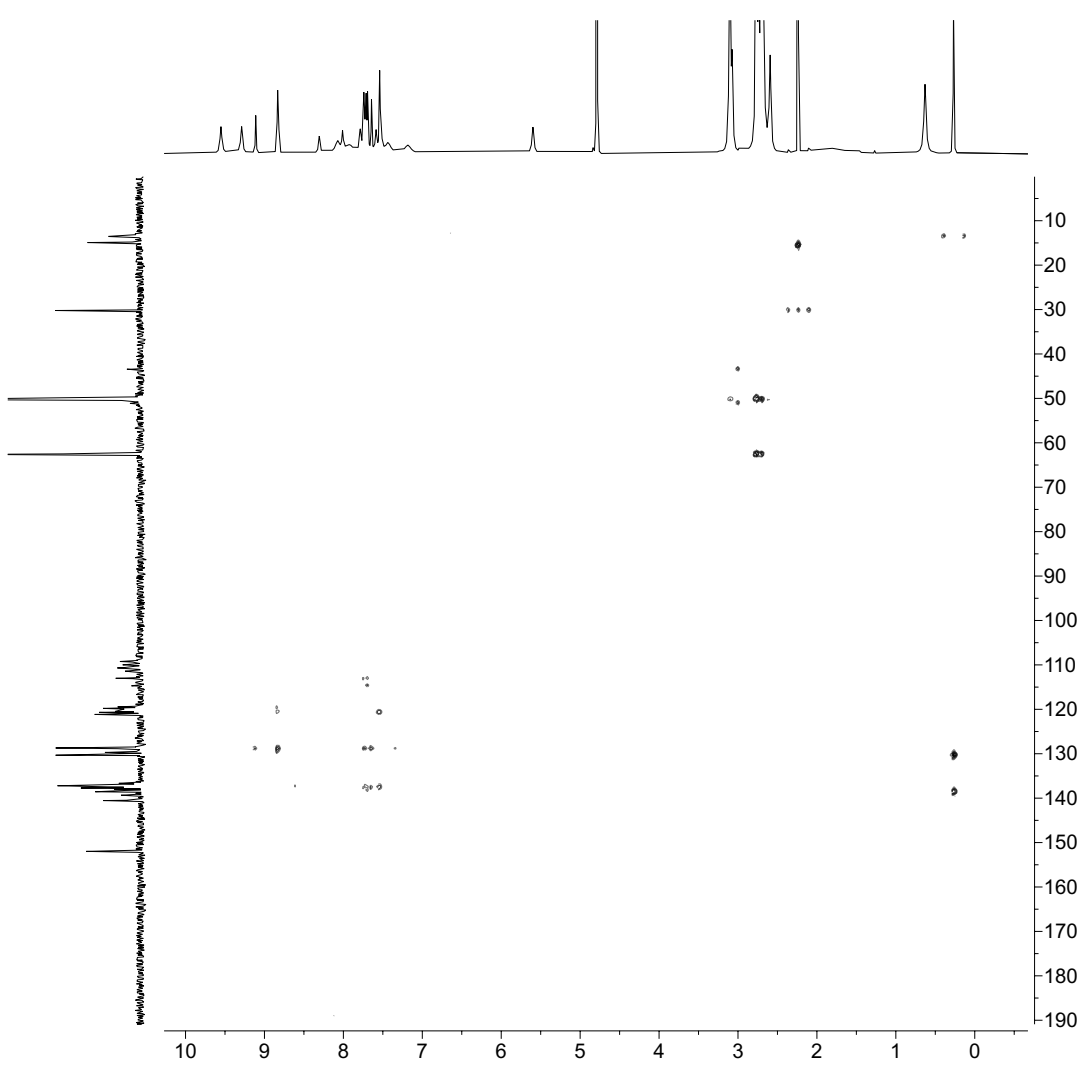

Figure S23. ${ }^{1} \mathrm{H}-{ }^{13} \mathrm{C}$ HMBC NMR spectrum of $\mathbf{2}_{2} \subset \mathbf{1}\left(500 \mathrm{MHz}, \mathrm{D}_{2} \mathrm{O}, 298 \mathrm{~K}\right)$.

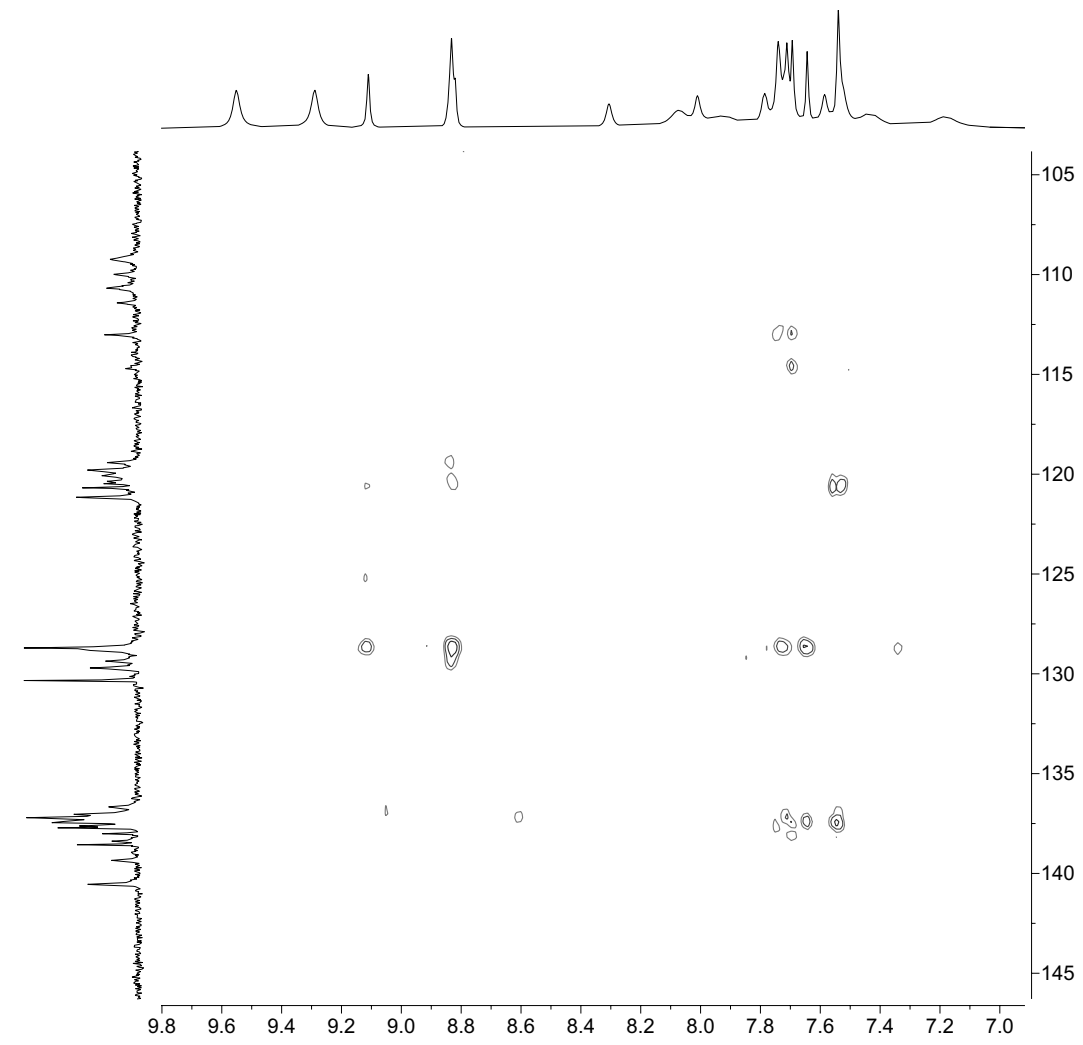

Figure S24. Partial ${ }^{1} \mathrm{H}-{ }^{13} \mathrm{C}$ HMBC NMR spectrum of $\mathbf{2}_{2} \subset \mathbf{1}\left(500 \mathrm{MHz}, \mathrm{D}_{2} \mathrm{O}, 298 \mathrm{~K}\right)$. 


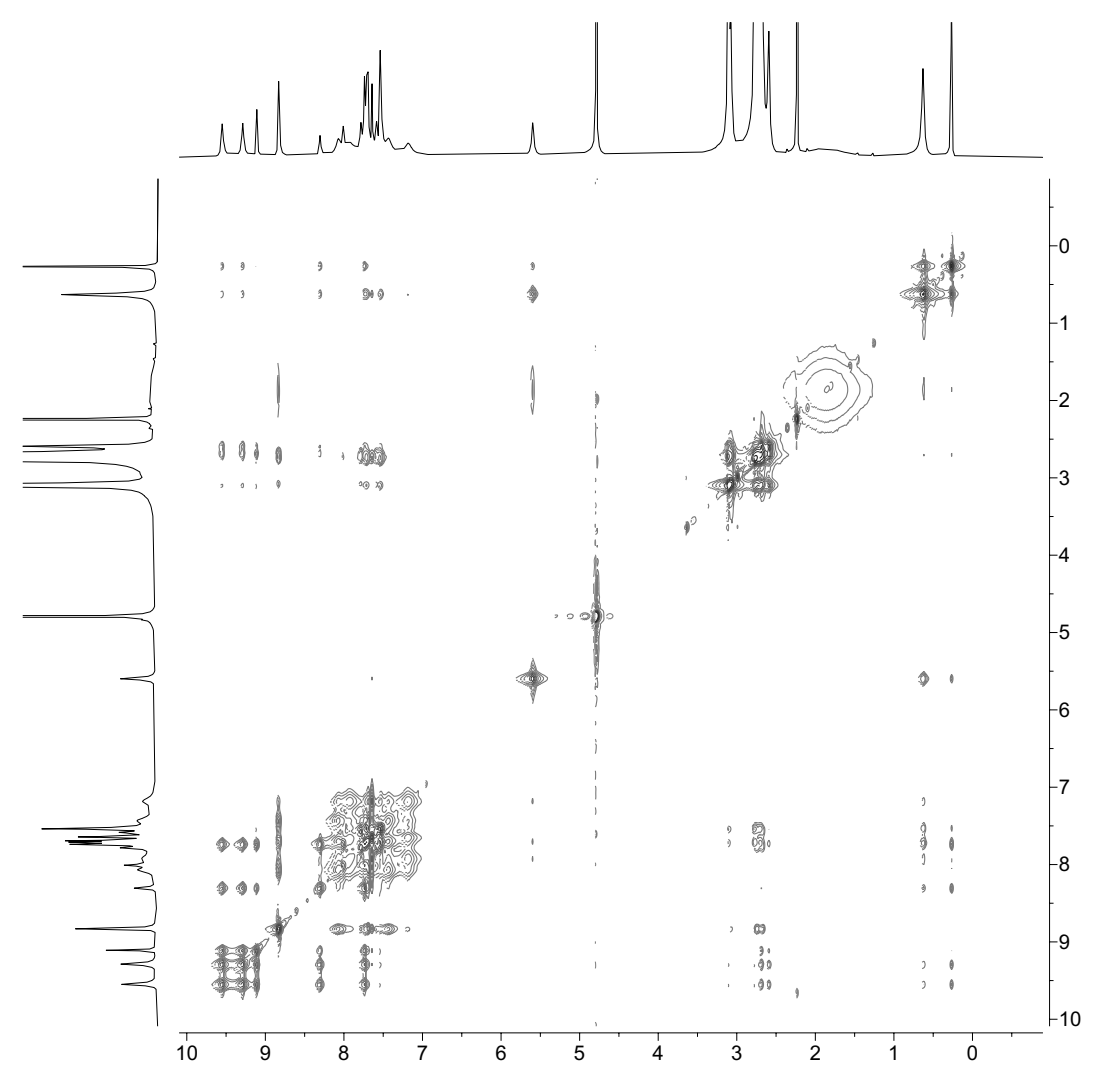

Figure S25. ${ }^{1} \mathrm{H}-{ }^{1} \mathrm{H}$ NOESY NMR spectrum of $\mathbf{2}_{2} \subset \mathbf{1}\left(500 \mathrm{MHz}, \mathrm{D}_{2} \mathrm{O}, 298 \mathrm{~K}\right)$.

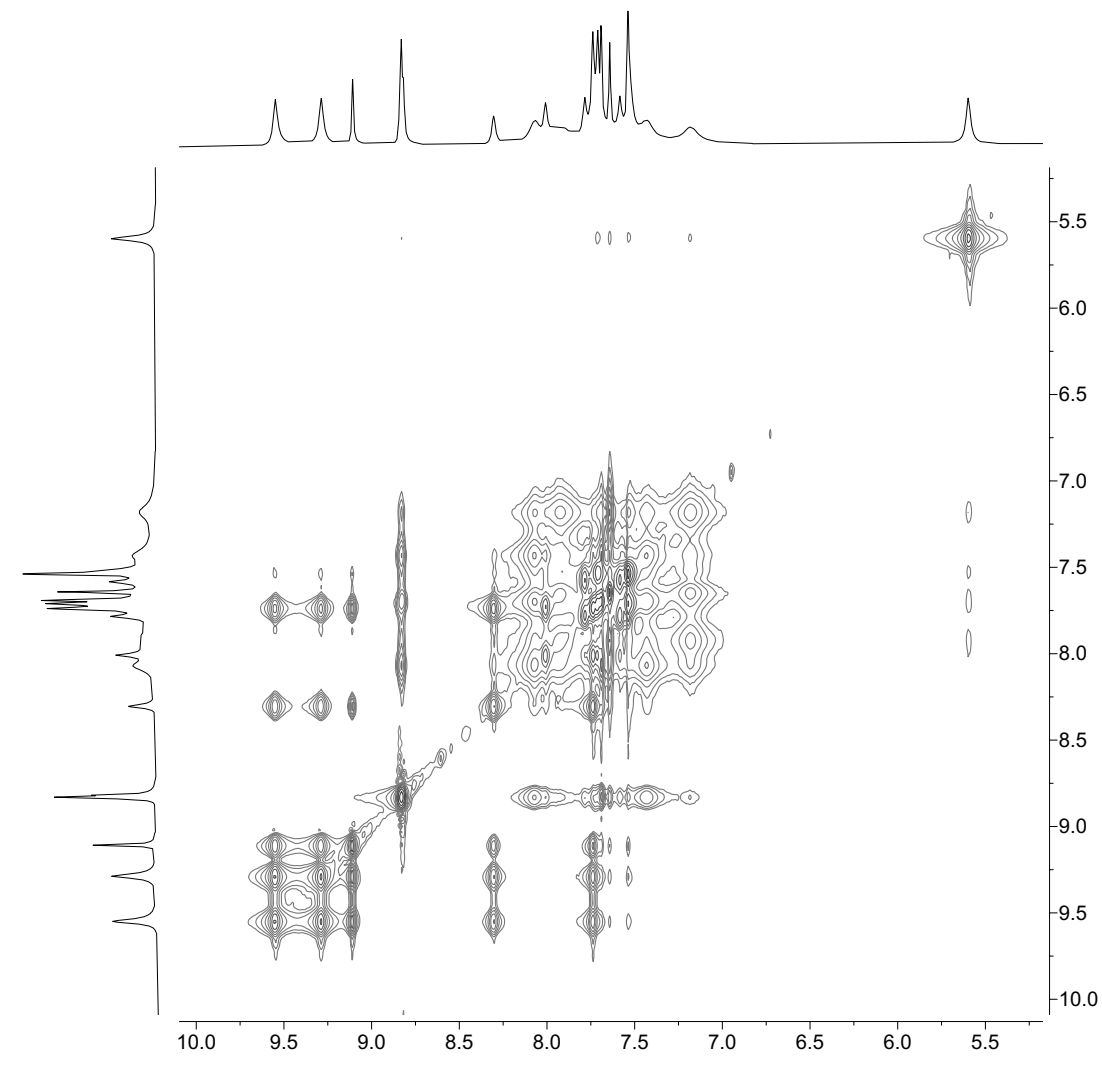

Figure S26. Partial ${ }^{1} \mathrm{H}-{ }^{1} \mathrm{H}$ NOESY NMR spectrum of $\mathbf{2}_{2} \subset \mathbf{1}$ showing nuclear Overhauser (nOe) correlations between 1 and 2 (500 MHz, $\left.\mathrm{D}_{2} \mathrm{O}, 298 \mathrm{~K}\right)$. 


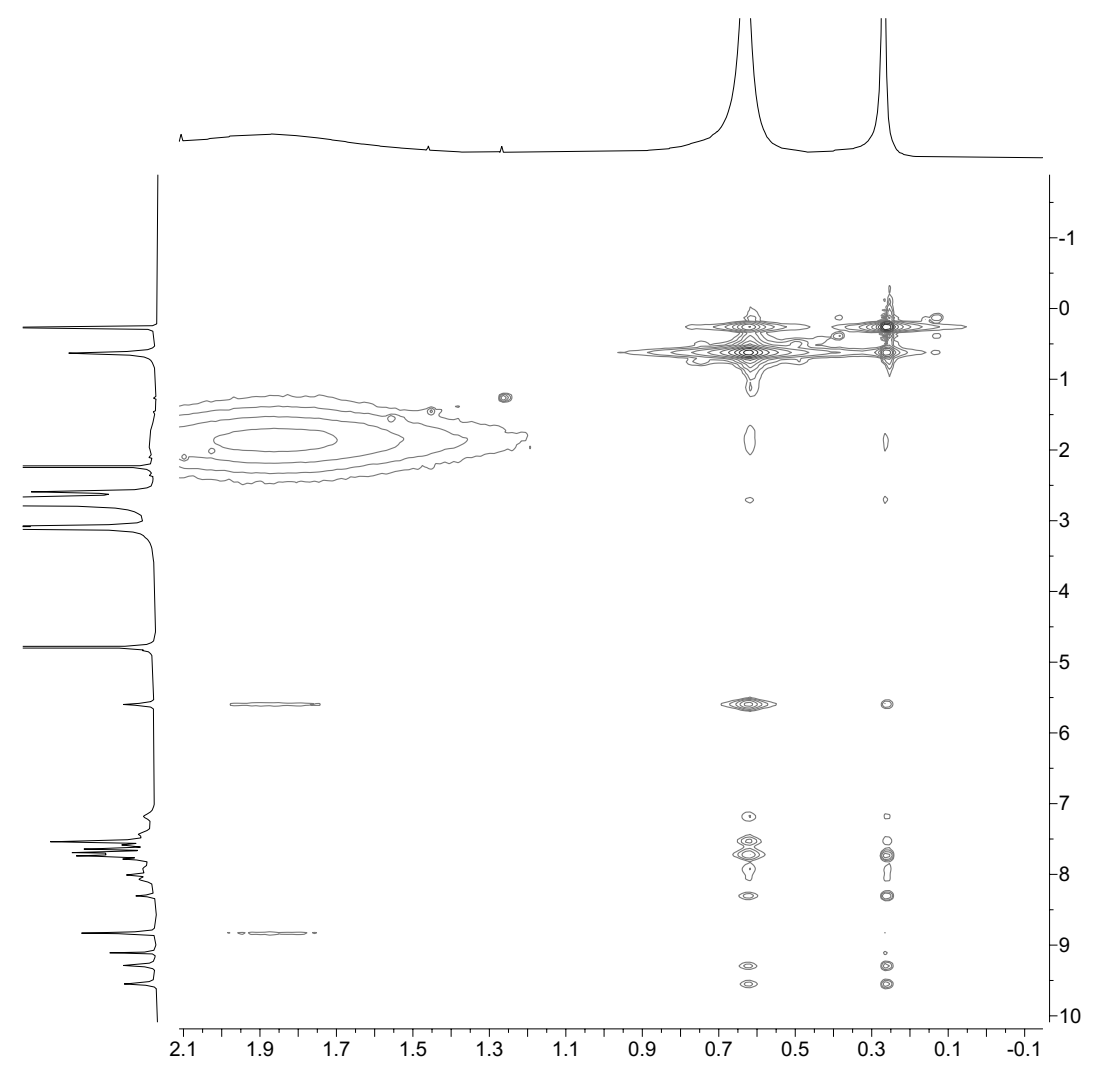

Figure S27. Partial ${ }^{1} \mathrm{H}-{ }^{1} \mathrm{H}$ NOESY NMR spectrum of $\mathbf{2}_{2} \subset \mathbf{1}$ showing nOe correlations between $\mathbf{1}$ and $\mathbf{2}$ and between two co-encapsulated molecules of $2\left(500 \mathrm{MHz}, \mathrm{D}_{2} \mathrm{O}, 298 \mathrm{~K}\right)$.

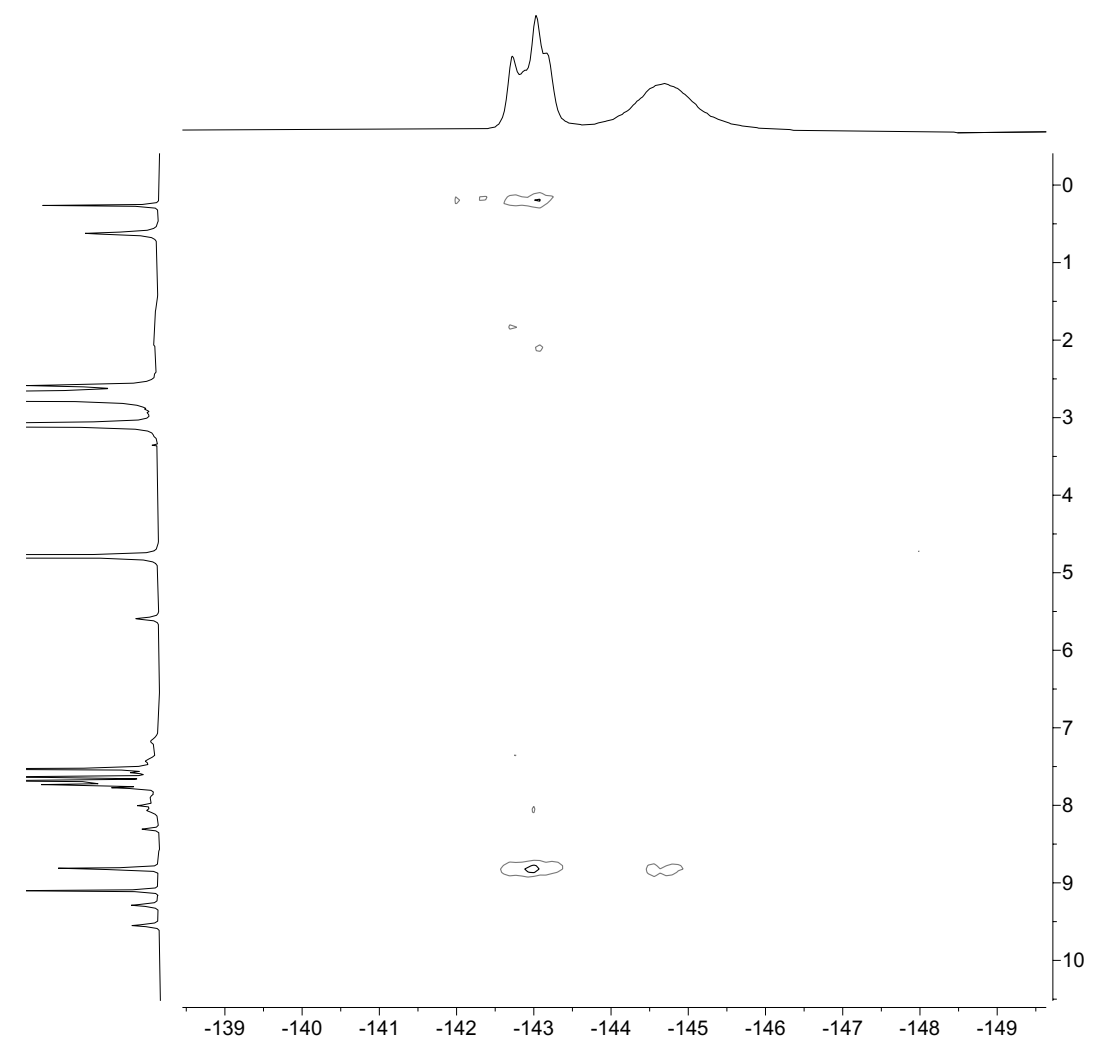

Figure S28. Partial ${ }^{1} \mathrm{H}-{ }^{19} \mathrm{~F}$ HOESY NMR spectrum of $\mathbf{2}_{2} \subset \mathbf{1}\left(500 \mathrm{MHz}, \mathrm{D}_{2} \mathrm{O}, 298 \mathrm{~K}\right)$. 
6. NMR characterization of inclusion complex $\mathbf{3}_{2} \subset \mathbf{1}$

Inclusion complex $\mathbf{3}_{2} \subset \mathbf{1}$ was obtained in a quantitative yield, as determined by ${ }^{1} \mathrm{H}$ NMR spectroscopy.

${ }^{1} \mathrm{H}$ NMR $\left(500 \mathrm{MHz}, \mathrm{D}_{2} \mathrm{O}\right): \delta=9.71\left(\mathrm{~s}, 4 \mathrm{H}, \mathbf{1}_{4}\right), 9.48\left(\mathrm{~s}, 4 \mathrm{H}, \mathbf{1}_{\mathbf{4}^{\prime}}\right), 8.84\left(\mathrm{~s}, 2 \mathrm{H}, \mathbf{1}_{\mathbf{1}}\right), 8.80\left(\mathrm{~s}, 2 \mathrm{H}, \mathbf{1}_{\mathbf{1}^{\prime}}\right), 8.57(\mathrm{~s}, 2 \mathrm{H}$,

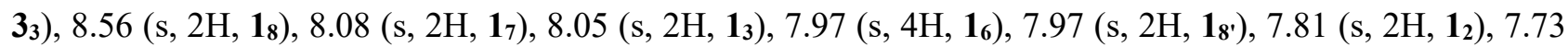
(s, 2H, 1 $\left.\mathbf{3}^{\prime}\right), 7.62\left(\mathrm{~s}, 4 \mathrm{H}, \mathbf{1}_{5}\right), 7.59\left(\mathrm{~s}, 2 \mathrm{H}, \mathbf{1}_{2^{\prime}}\right), 7.51\left(\mathrm{~s}, 4 \mathrm{H}, \mathbf{1}_{5^{\prime}}\right), 7.36\left(\mathrm{br}, 4 \mathrm{H}, \mathbf{1}_{7^{\prime}}\right), 7.17\left(\mathrm{br}, 4 \mathrm{H}, \mathbf{1}_{6^{\prime}}\right), 3.09(\mathrm{~s}, 24 \mathrm{H}$, $\left.\mathbf{1}_{11}\right), 2.84-2.52\left(\mathrm{~m}, 72 \mathrm{H}, \mathbf{1}_{12}\right), 2.24\left(\mathrm{br}, 12 \mathrm{H}, \mathbf{3}_{1}\right), 1.58\left(\mathrm{~s}, 12 \mathrm{H}, \mathbf{3}_{4}\right),-0.06\left(\mathrm{~s}, 12 \mathrm{H}, \mathbf{3}_{2}\right)$.

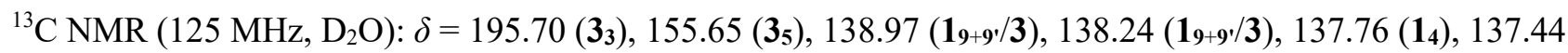
$\left(\mathbf{1}_{\left.\mathbf{4}^{\prime}+\mathbf{1}^{\prime}+\mathbf{1 0}\right)}\right), 136.80\left(\mathbf{1}_{\mathbf{1 0}^{\prime}}\right), 136.58\left(\mathbf{1}_{\mathbf{1}}\right), 136.09\left(\mathbf{1}_{\mathbf{9}+\mathbf{9}^{\prime}} / \mathbf{3}\right), 132.19\left(\mathbf{3}_{\mathbf{8}}\right), 129.56\left(\mathbf{1}_{\mathbf{5}}\right), 129.46\left(\mathbf{1}_{\mathbf{2}}\right), 128.63\left(\mathbf{1}_{\mathbf{2}^{\prime}}\right), 128.54$ $\left(\mathbf{1}_{5^{\prime}}\right), 126.95\left(\mathbf{3}_{4}\right), 126.38(\mathbf{3}), 120.25\left(\mathbf{1}_{\mathbf{3}}\right), 119.24\left(\mathbf{1}_{\mathbf{6}^{+} \mathbf{6}^{\prime}}\right), 119.16\left(\mathbf{1}_{3^{\prime}}\right), 111.92\left(\mathbf{1}_{\mathbf{8}}\right), 110.68\left(\mathbf{1}_{\mathbf{8}^{\prime}}\right), 109.57\left(\mathbf{1}_{7}\right)$, $107.62\left(\mathbf{1}_{7^{\prime}}\right), 62.66\left(\mathbf{1}_{11}\right), 50.41\left(\mathbf{1}_{12}\right), 50.22\left(\mathbf{1}_{12}\right), 12.14(\mathbf{3}), 10.37(\mathbf{3}), 7.90(\mathbf{3})$.

${ }^{19} \mathrm{~F}$ NMR (470 MHz, $\left.\mathrm{D}_{2} \mathrm{O}\right): \delta=(-143.0)-(-144.0)(\mathrm{m}, 2 \mathrm{~F}, 3),-145.0(\mathrm{br}, 2 \mathrm{~F}, 3)$.

${ }^{1} \mathrm{H}$ DOSY NMR $\left(500 \mathrm{MHz}, \mathrm{D}_{2} \mathrm{O}\right): D=0.15 \times 10^{-5} \mathrm{~cm}^{2} / \mathrm{s}$.

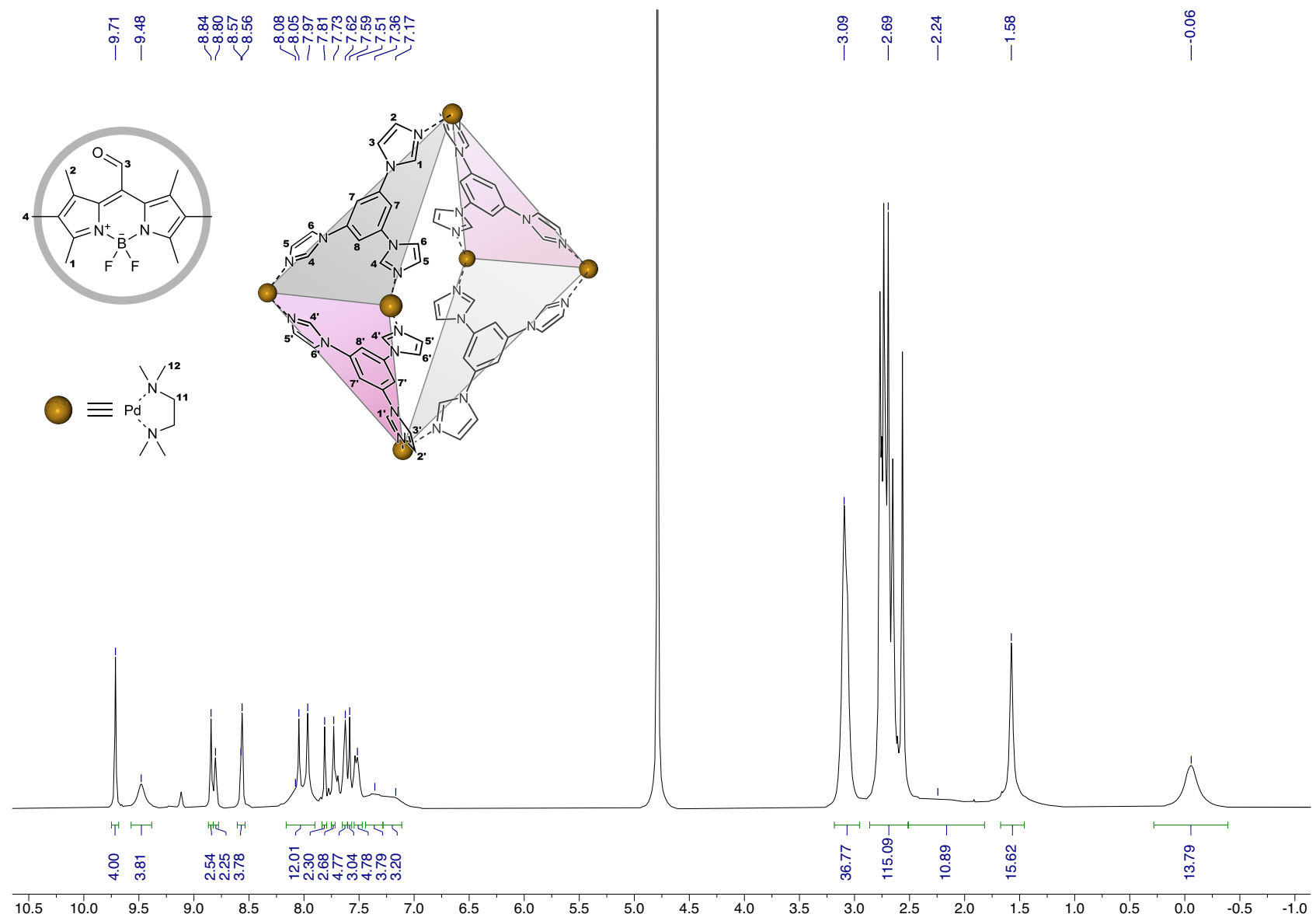

Figure S29. ${ }^{1} \mathrm{H}$ NMR spectrum of $\mathbf{3}_{2} \subset \mathbf{1}\left(500 \mathrm{MHz}, \mathrm{D}_{2} \mathrm{O}, 298 \mathrm{~K}\right)$. 


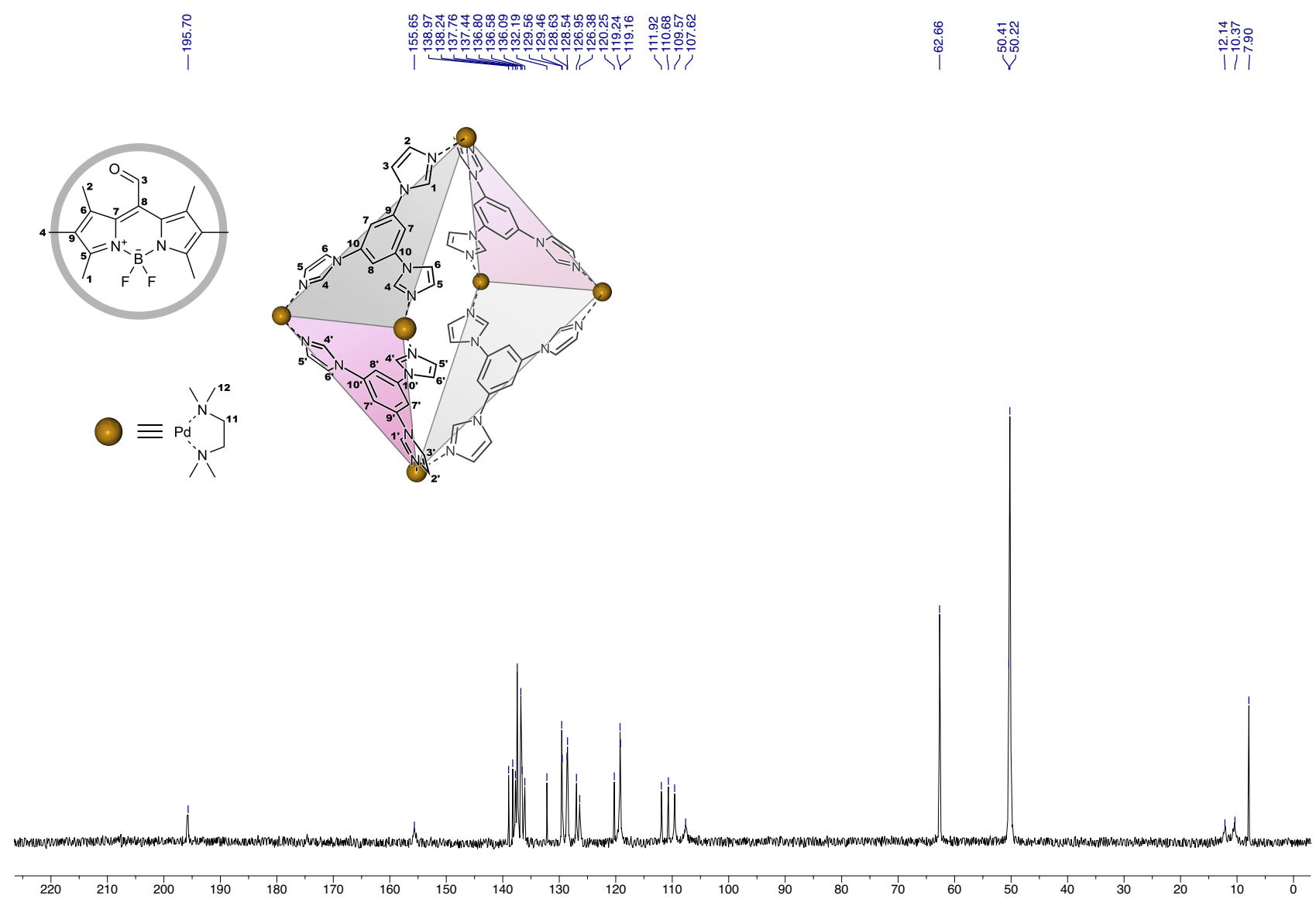

Figure S30. ${ }^{13} \mathrm{C}$ NMR spectrum of $\mathbf{3}_{2} \subset \mathbf{1}\left(125 \mathrm{MHz}, \mathrm{D}_{2} \mathrm{O}, 298 \mathrm{~K}\right)$.

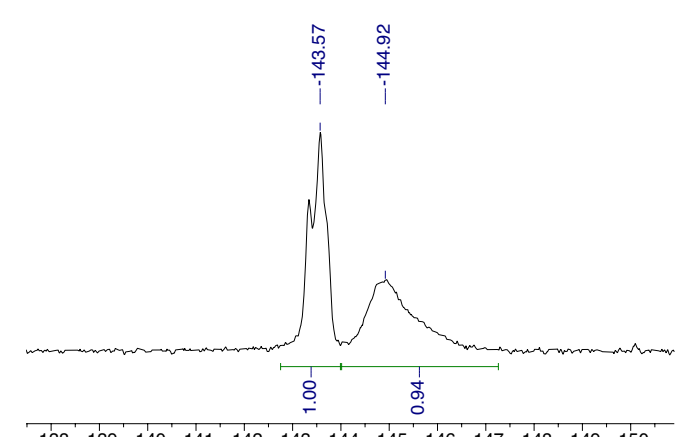

Figure S31. ${ }^{19} \mathrm{~F}$ NMR spectrum of $\mathbf{3}_{2} \subset \mathbf{1}$ ( $470 \mathrm{MHz}, \mathrm{D}_{2} \mathrm{O}, 298 \mathrm{~K}$ ). 


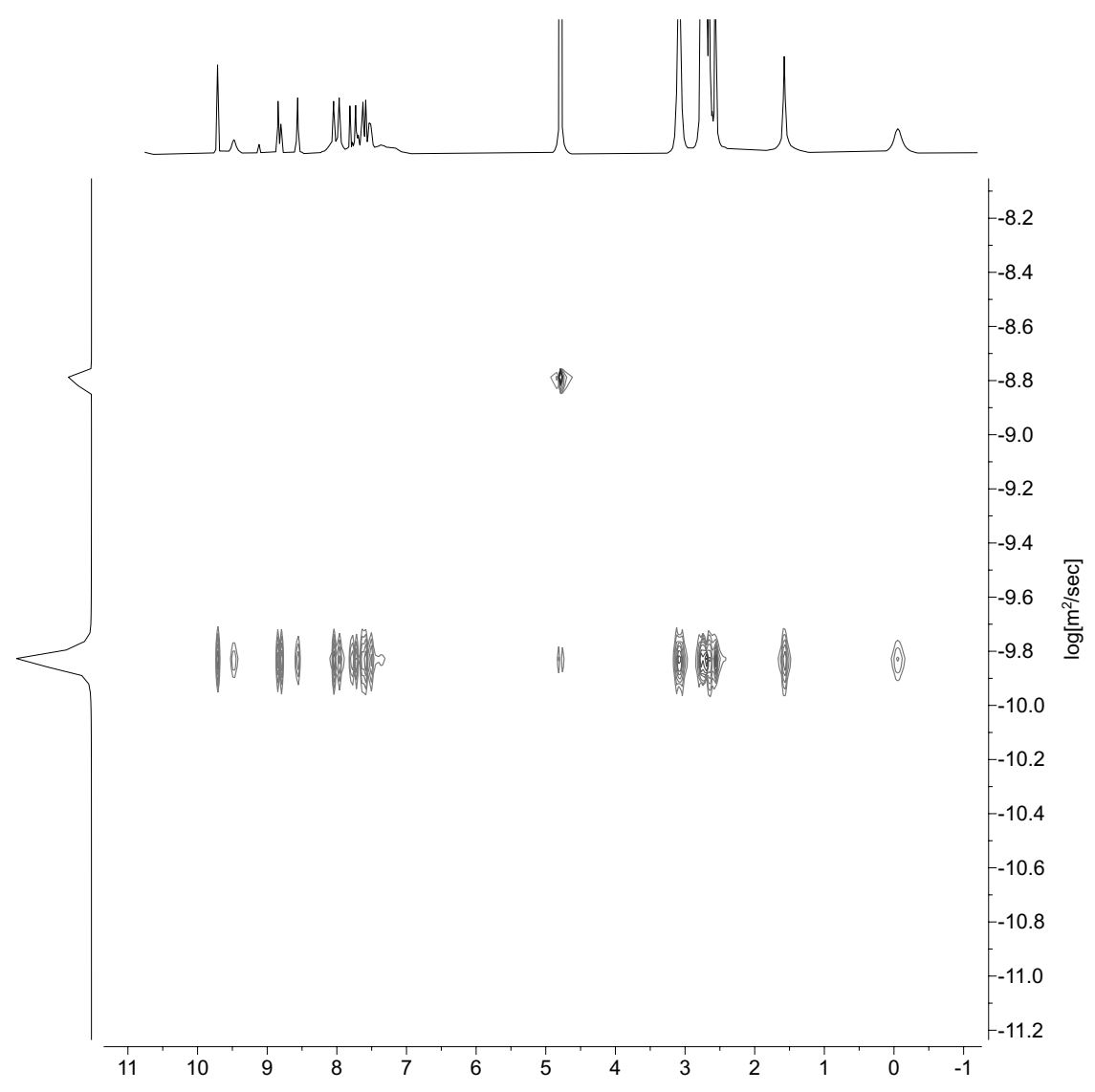

Figure S32. ${ }^{1} \mathrm{H}$ DOSY NMR spectrum of $\mathbf{3}_{2} \subset \mathbf{1}\left(500 \mathrm{MHz}, \mathrm{D}_{2} \mathrm{O}, 298 \mathrm{~K}\right)$.

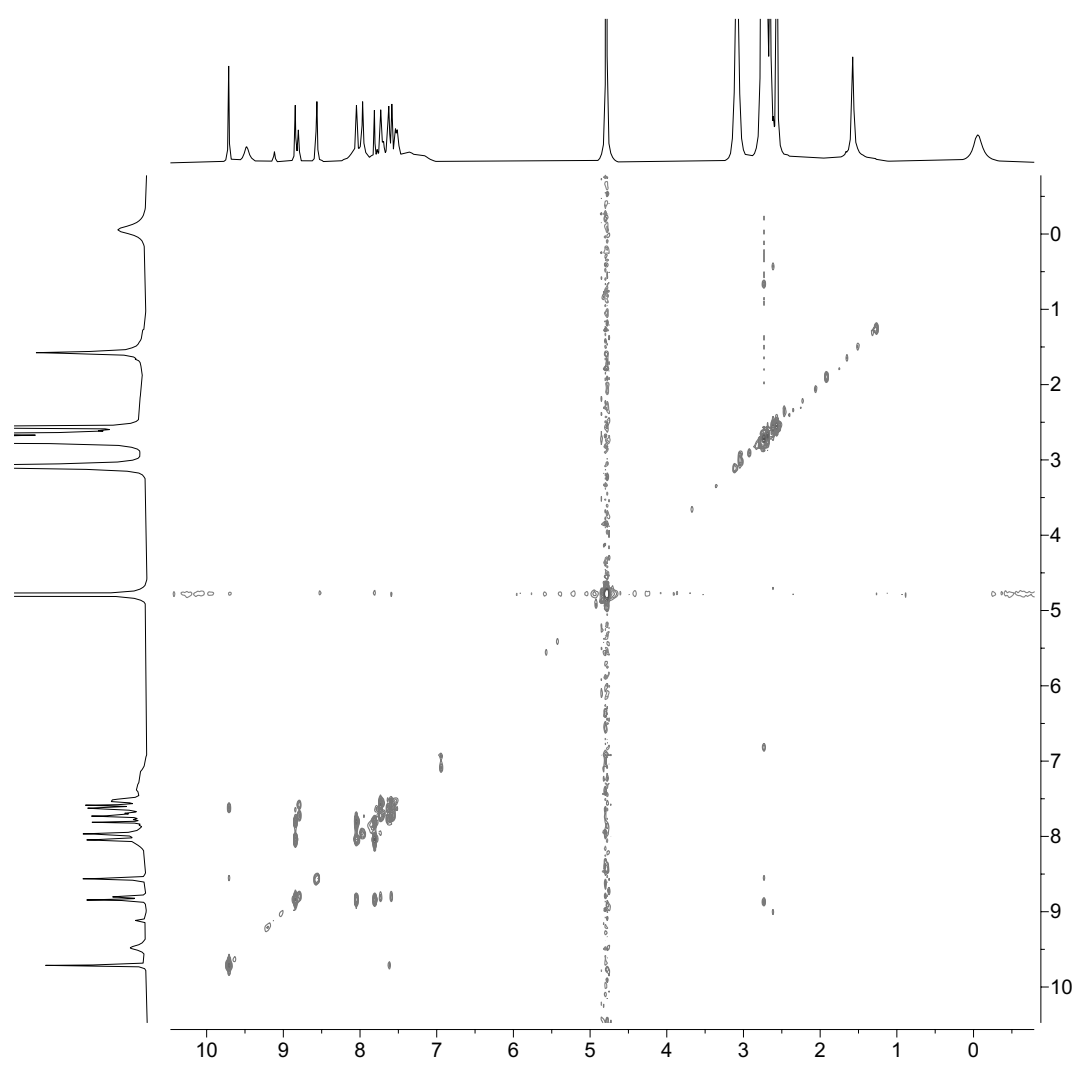

Figure S33. ${ }^{1} \mathrm{H}-{ }^{1} \mathrm{H}$ COSY NMR spectrum of $\mathbf{3}_{2} \subset \mathbf{1}\left(500 \mathrm{MHz}, \mathrm{D}_{2} \mathrm{O}, 298 \mathrm{~K}\right)$. 


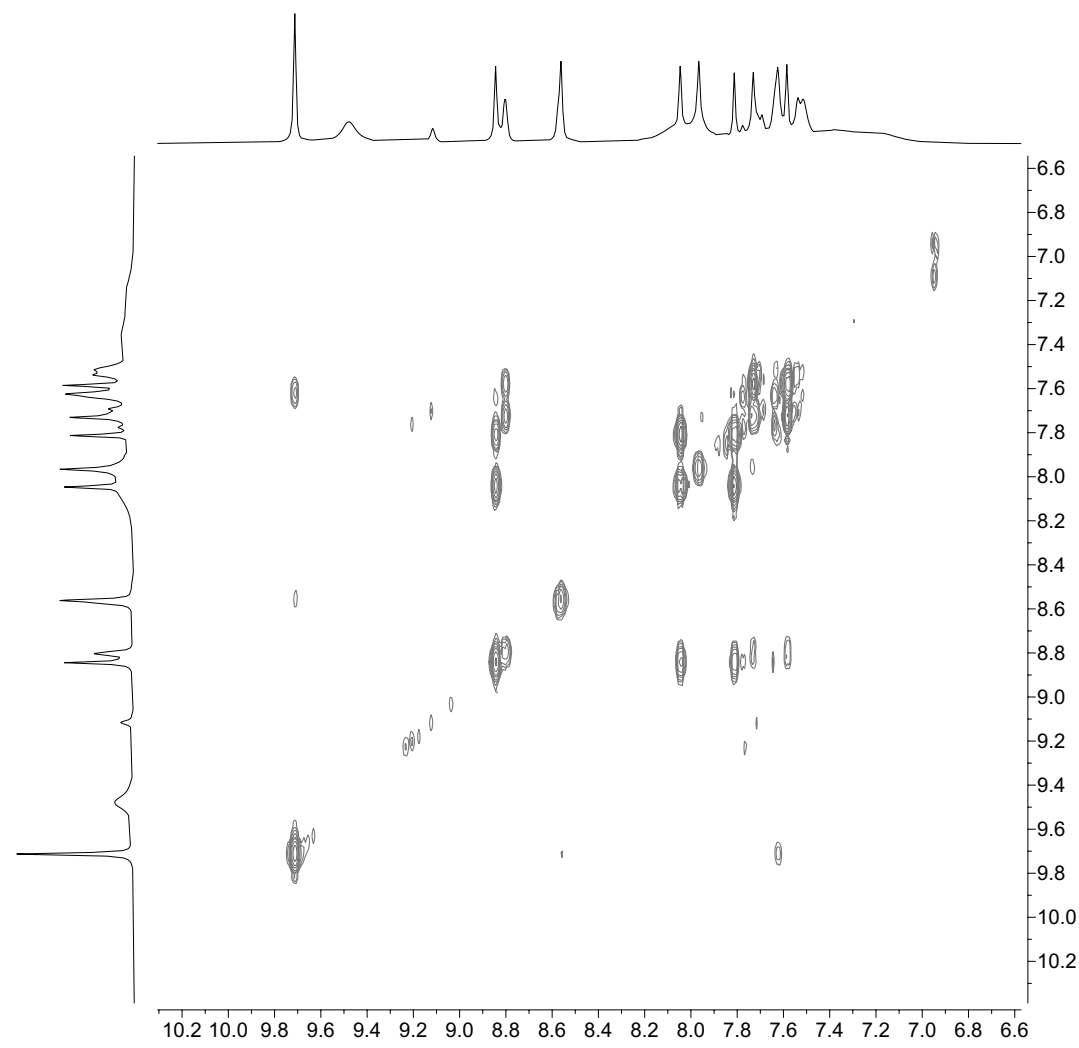

Figure S34. Partial ${ }^{1} \mathrm{H}-{ }^{1} \mathrm{H}$ COSY NMR spectrum of $\mathbf{3}_{2} \subset \mathbf{1}\left(500 \mathrm{MHz}, \mathrm{D}_{2} \mathrm{O}, 298 \mathrm{~K}\right)$.

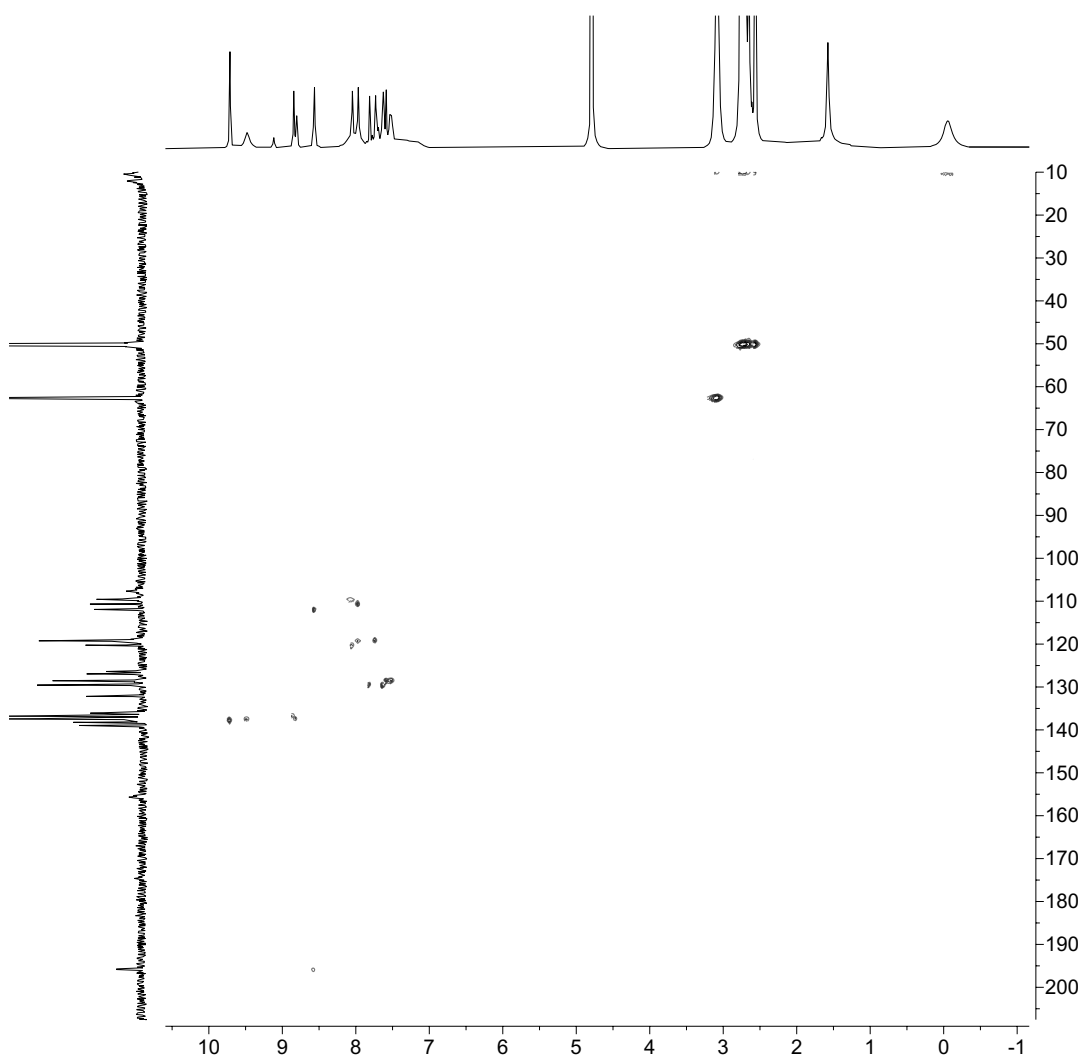

Figure S35. ${ }^{1} \mathrm{H}-{ }^{13} \mathrm{C}$ HSQC NMR spectrum of $\mathbf{3}_{2} \subset \mathbf{1}\left(500 \mathrm{MHz}, \mathrm{D}_{2} \mathrm{O}, 298 \mathrm{~K}\right)$. 


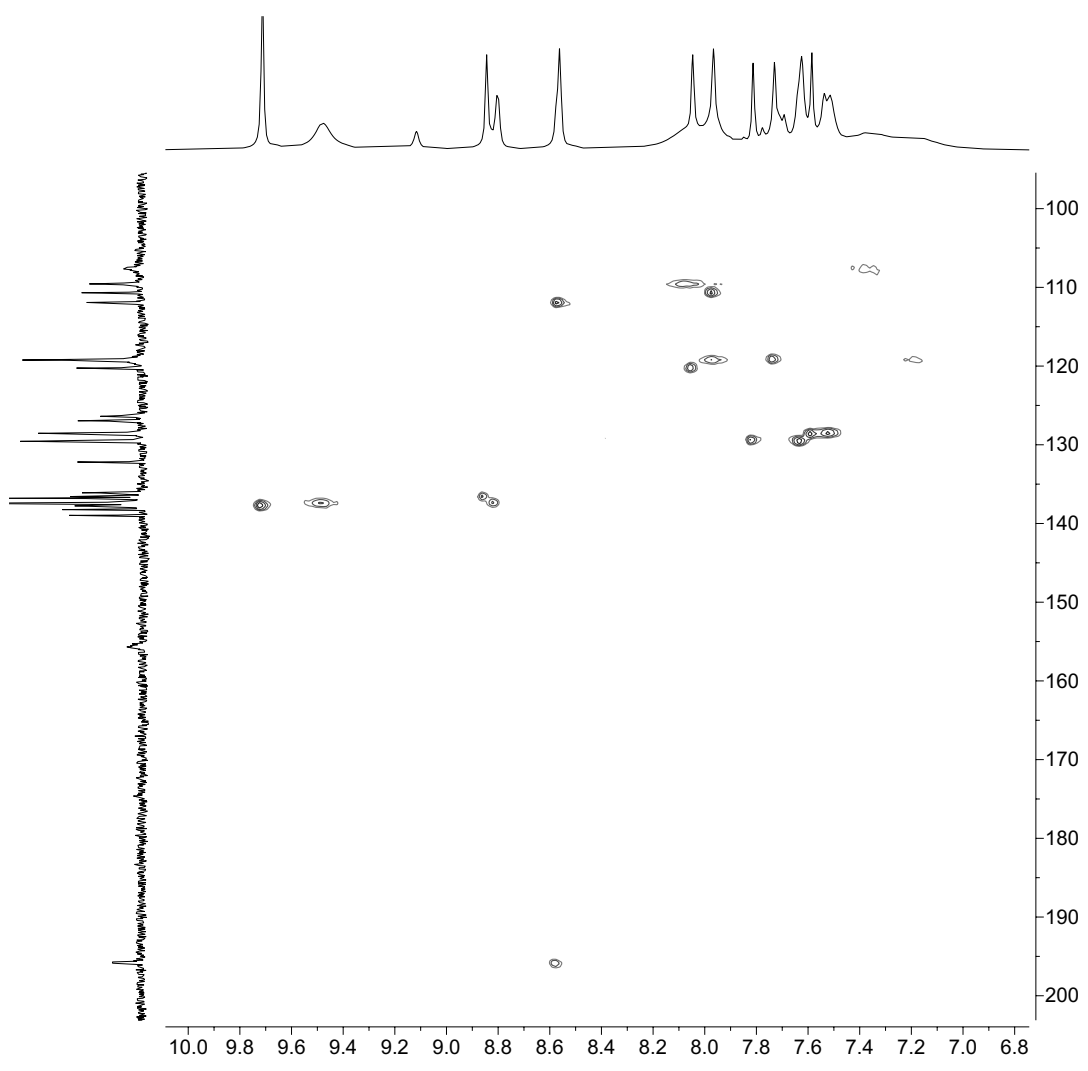

Figure S36. Partial ${ }^{1} \mathrm{H}-{ }^{13} \mathrm{C}$ HSQC NMR spectrum of $\mathbf{3}_{2} \subset \mathbf{1}\left(500 \mathrm{MHz}, \mathrm{D}_{2} \mathrm{O}, 298 \mathrm{~K}\right)$.

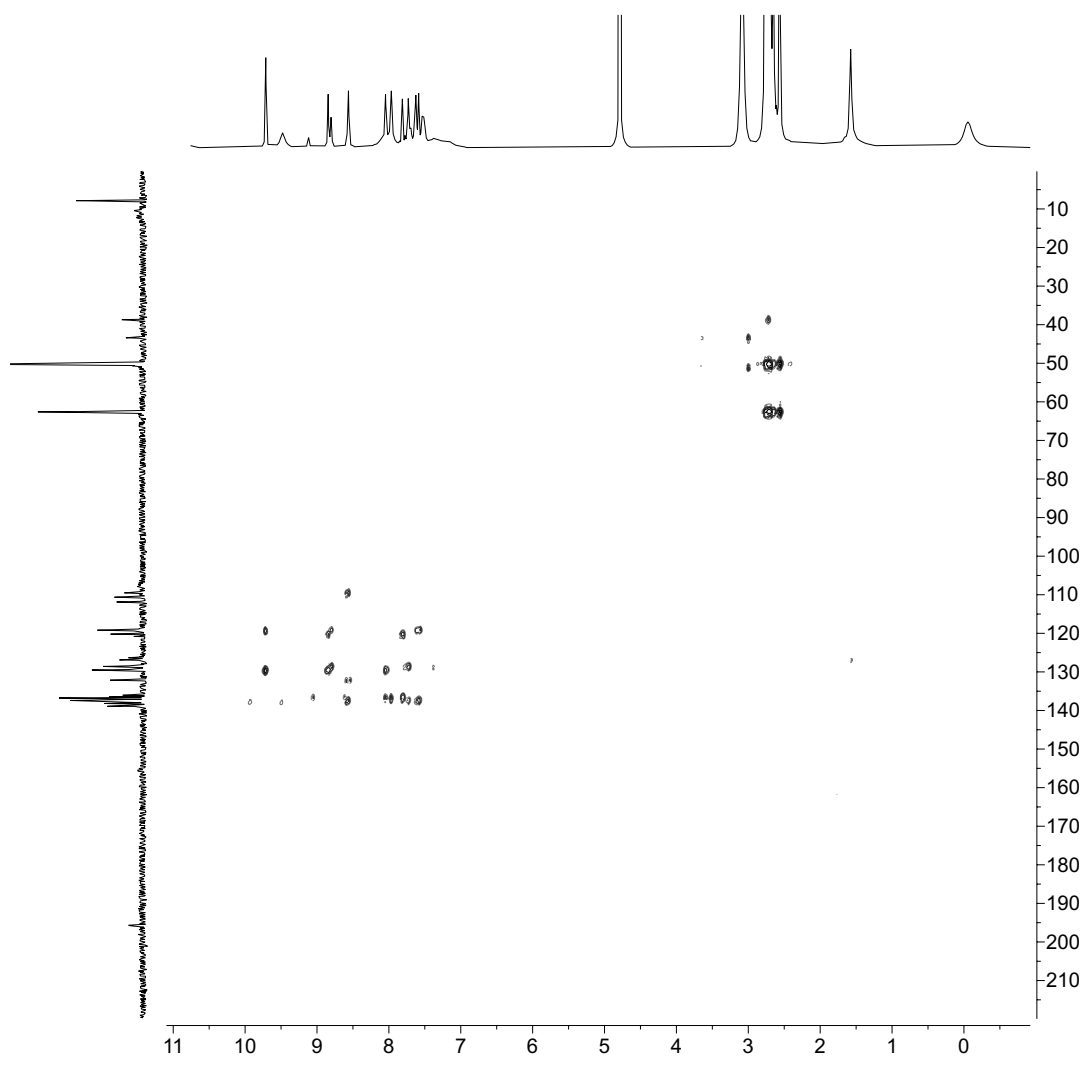

Figure S37. ${ }^{1} \mathrm{H}-{ }^{13} \mathrm{C}$ HMBC NMR spectrum of $\mathbf{3}_{2} \subset \mathbf{1}\left(500 \mathrm{MHz}, \mathrm{D}_{2} \mathrm{O}, 298 \mathrm{~K}\right)$. 


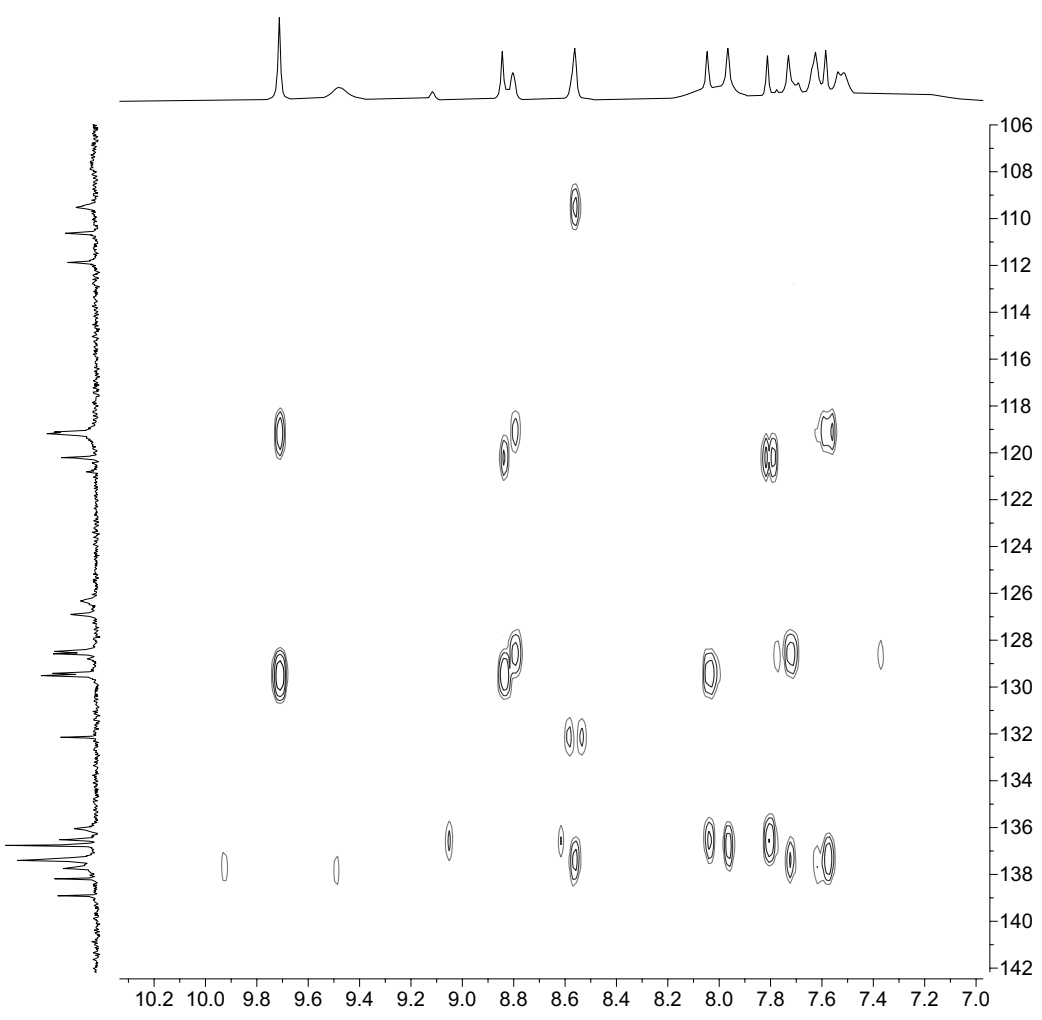

Figure S38. Partial ${ }^{1} \mathrm{H}-{ }^{13} \mathrm{C}$ HMBC NMR spectrum of $\mathbf{3}_{2} \subset \mathbf{1}\left(500 \mathrm{MHz}, \mathrm{D}_{2} \mathrm{O}, 298 \mathrm{~K}\right)$. 


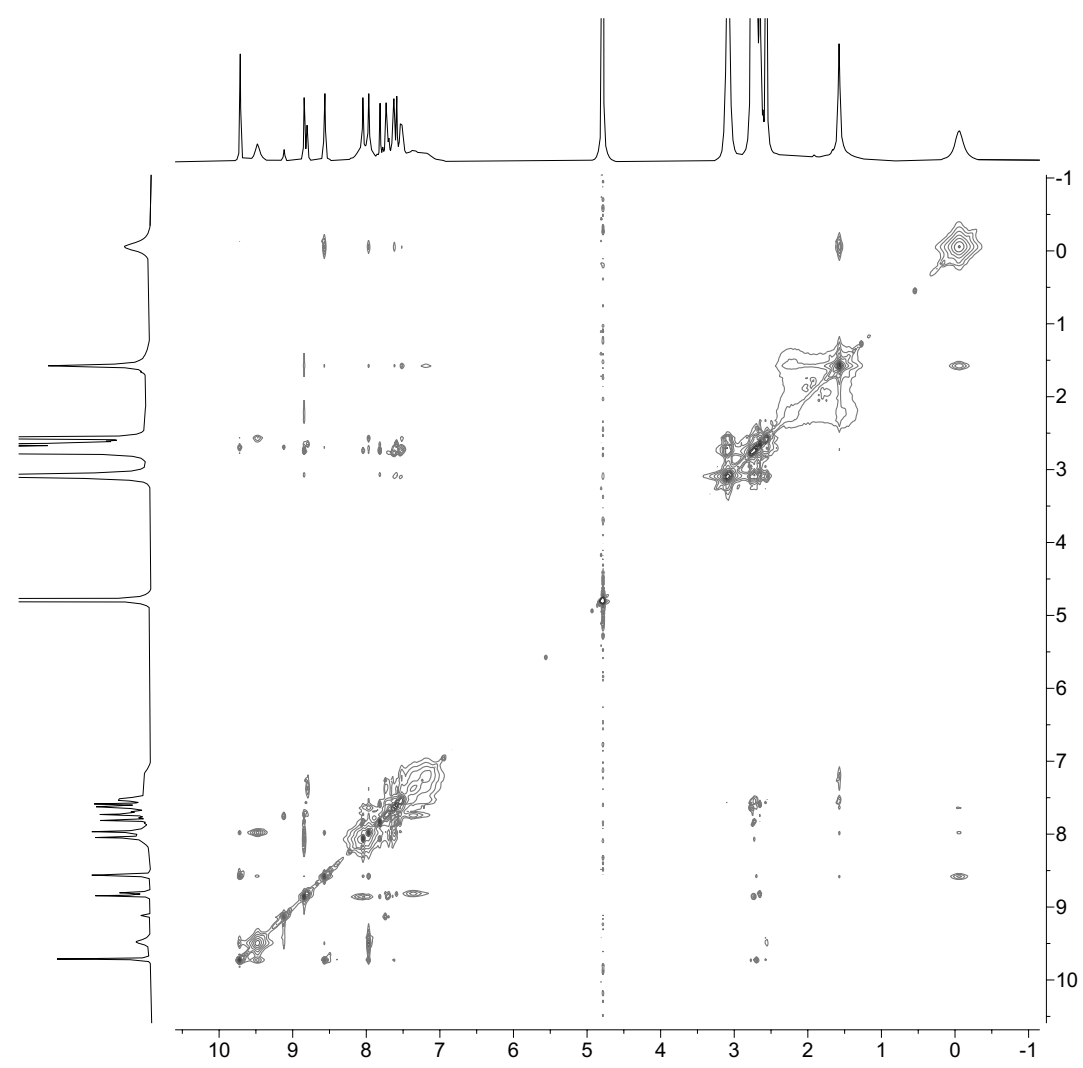

Figure S39. ${ }^{1} \mathrm{H}-{ }^{1} \mathrm{H}$ NOESY NMR spectrum of $\mathbf{3}_{2} \subset \mathbf{1}\left(500 \mathrm{MHz}, \mathrm{D}_{2} \mathrm{O}, 298 \mathrm{~K}\right)$.

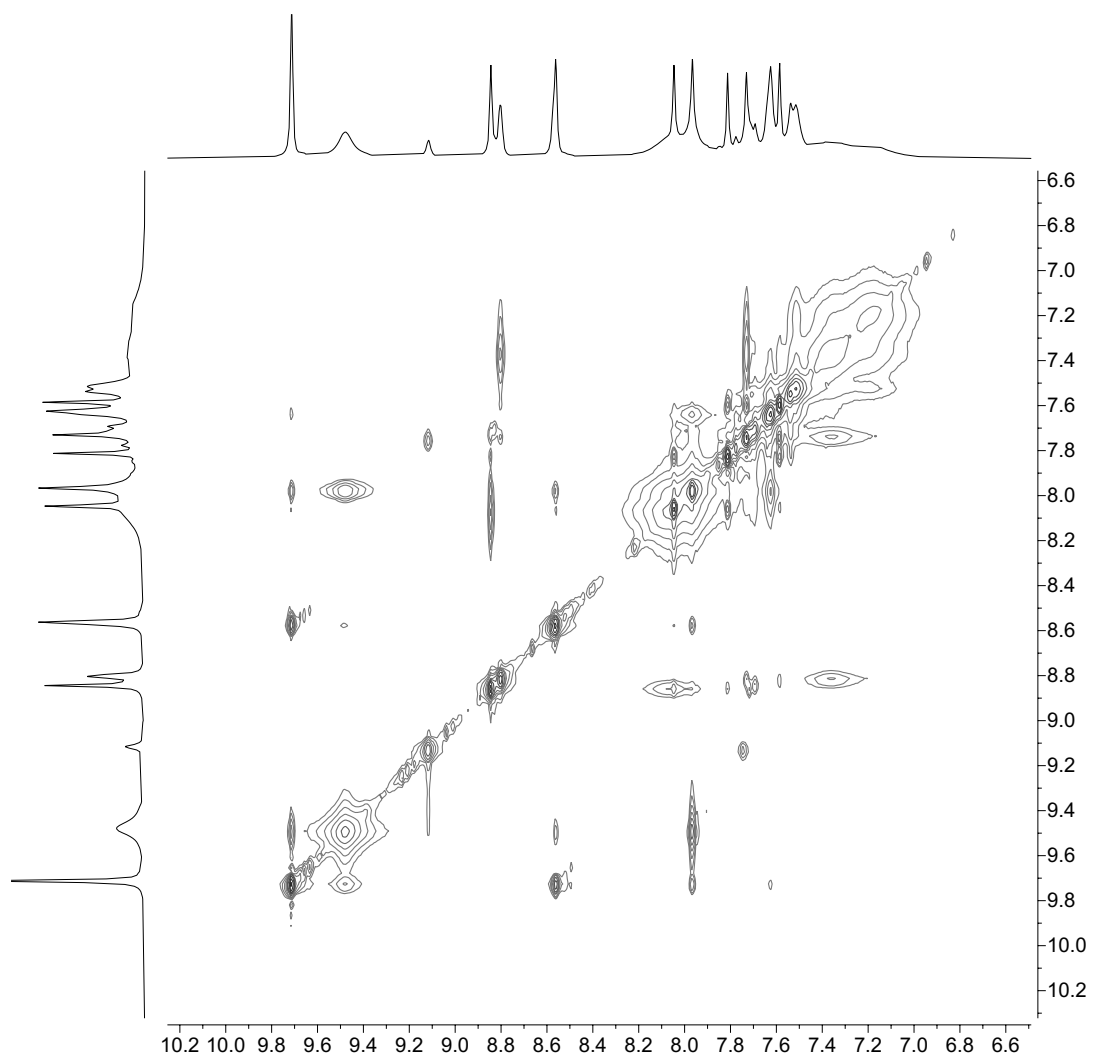

Figure S40. Partial ${ }^{1} \mathrm{H}-{ }^{1} \mathrm{H}$ NOESY NMR spectrum of $\mathbf{3}_{2} \subset \mathbf{1}$ showing nOe correlations between $\mathbf{1}$ and $\mathbf{3}$ (500 $\left.\mathrm{MHz}, \mathrm{D}_{2} \mathrm{O}, 298 \mathrm{~K}\right)$. 


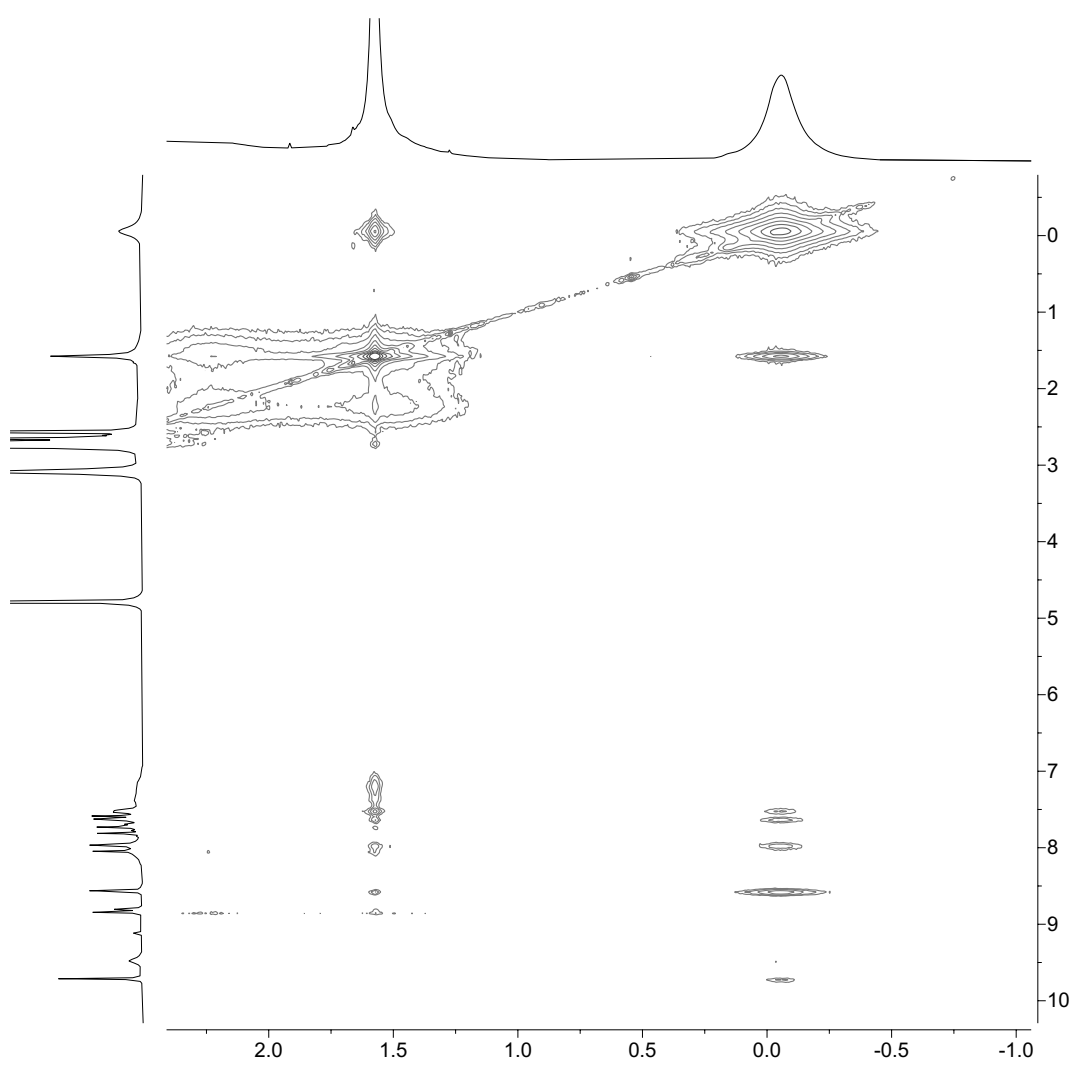

Figure S41. Partial ${ }^{1} \mathrm{H}-{ }^{1} \mathrm{H}$ NOESY NMR spectrum of $\mathbf{3}_{2} \subset \mathbf{1}$ showing nOe correlations between $\mathbf{1}$ and $\mathbf{3}$ and between two co-encapsulated molecules of $\mathbf{3}\left(500 \mathrm{MHz}, \mathrm{D}_{2} \mathrm{O}, 298 \mathrm{~K}\right)$. 


\section{NMR characterization of inclusion complex $\mathbf{4}_{2} \subset \mathbf{1}$}

Inclusion complex $\mathbf{4}_{2} \subset \mathbf{1}$ was obtained in $\sim 62 \%$ yield, as determined by UV-vis absorption spectroscopy (see Section 9). The signals in the NMR spectra of $\mathbf{4}_{2} \subset \mathbf{1}$ were significantly broader than those of the other two inclusion complexes, which made the analysis of $\mathbf{4}_{2} \subset \mathbf{1}$ using 2D NMR methods challenging.

${ }^{1} \mathrm{H}$ NMR (500 MHz, $\mathrm{D}_{2} \mathrm{O}$ ): $\delta=9.59$ (s), 9.34 (s), 9.13-9.05 (br), 8.15-7.77 (br), 5.98 (s), 3.31 (s), 3.29 (s), $2.97(\mathrm{~s}), 2.87(\mathrm{~s}), 1.62(\mathrm{br}), 1.01(\mathrm{~s})$.

${ }^{13} \mathrm{C}$ NMR $\left(125 \mathrm{MHz}, \mathrm{D}_{2} \mathrm{O}\right): \delta=137.81,137.39,129.11,128.80,124.63,121.87,120.97,119.66,119.14$, $109.27,62.60,50.27,14.06$.

${ }^{19} \mathrm{~F}$ NMR (470 MHz, $\left.\mathrm{D}_{2} \mathrm{O}\right): \delta=-55.3\left(\mathrm{~s}, 6 \mathrm{~F}, \mathbf{3}_{\mathrm{CF} 3}\right)(-138.3)-(-143.3)\left(\mathrm{m}, 2 \mathrm{~F}, \mathbf{3}_{\mathrm{BF} 2}\right),-145.0\left(\mathrm{~m}, 2 \mathrm{~F}, \mathbf{3}_{\mathrm{BF} 2}\right)$.

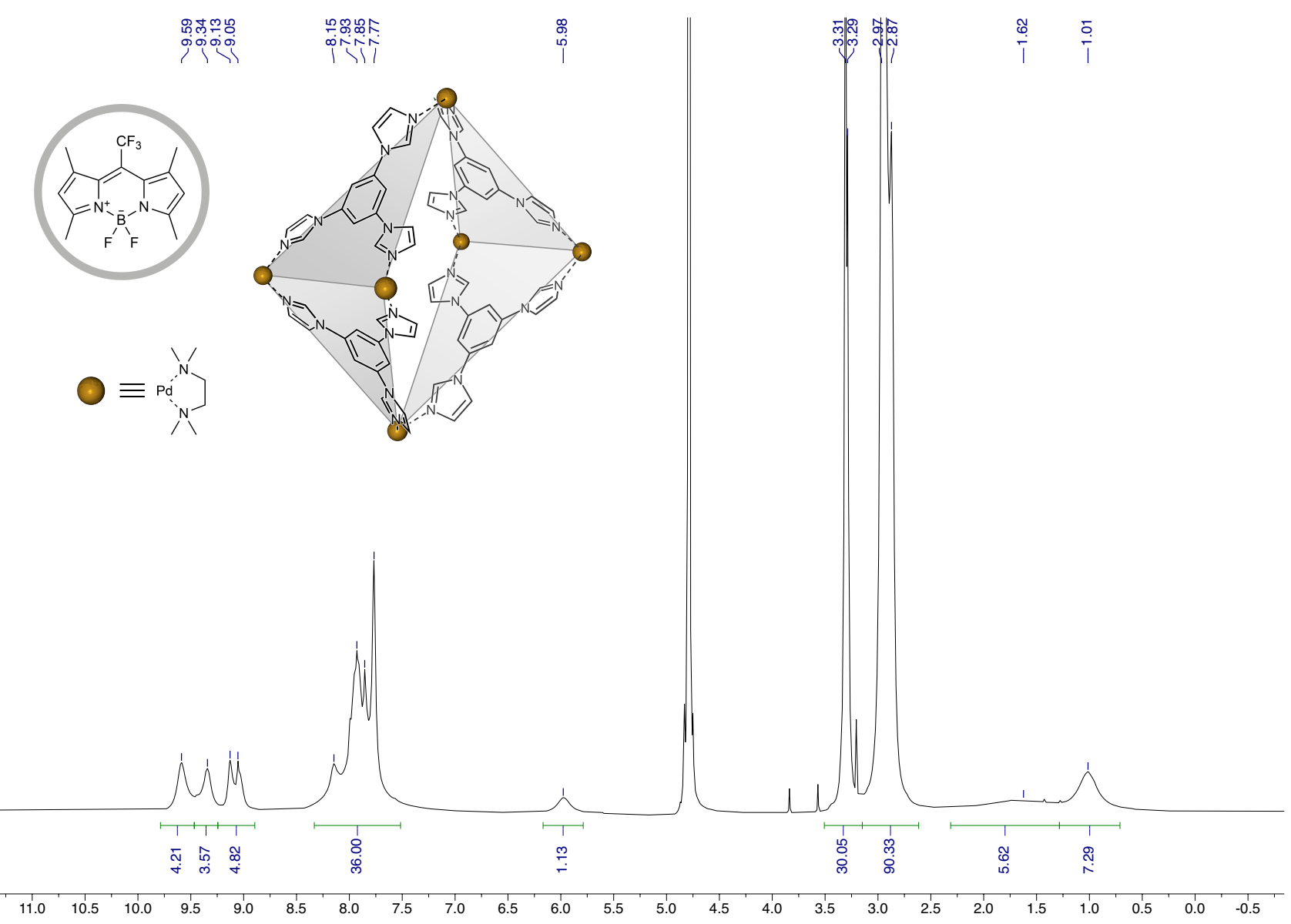

Figure S42. ${ }^{1} \mathrm{H}$ NMR spectrum of $\mathbf{4}_{2} \subset \mathbf{1}\left(500 \mathrm{MHz}, \mathrm{D}_{2} \mathrm{O}, 318 \mathrm{~K}\right)$. Note that the spectrum was recorded at 318 $\mathrm{K}$; the peaks at room temperature were significantly broader. 


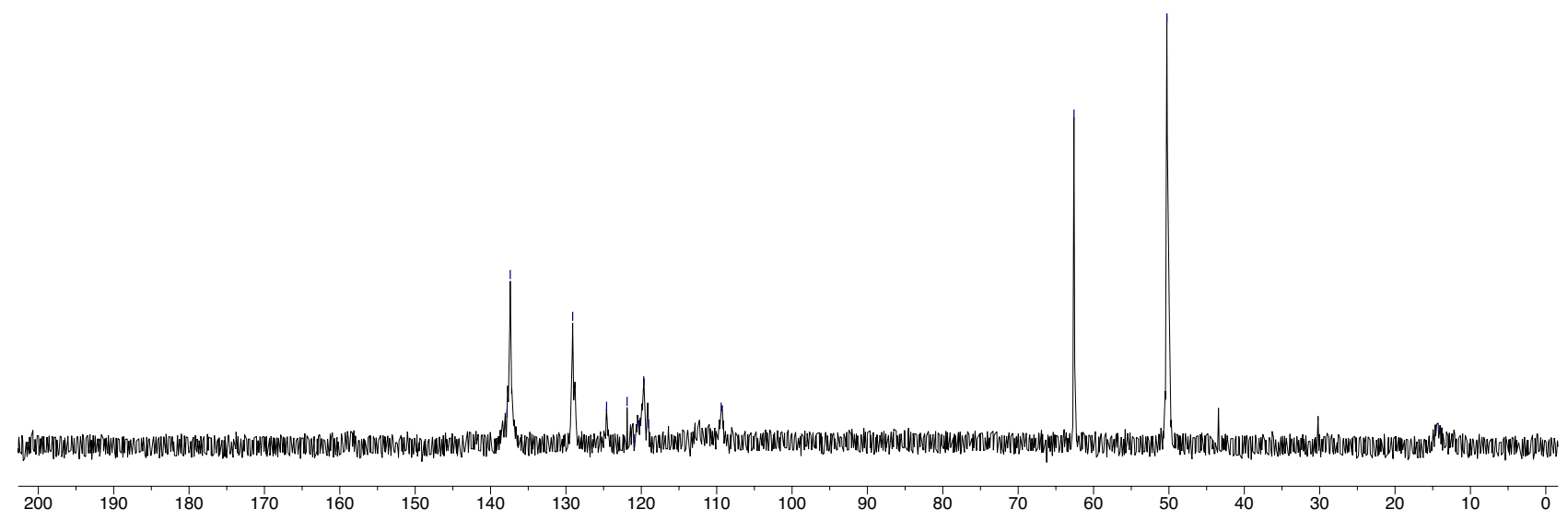

Figure S43. ${ }^{13} \mathrm{C}$ NMR spectrum of $\mathbf{4}_{2} \subset \mathbf{1}\left(125 \mathrm{MHz}, \mathrm{D}_{2} \mathrm{O}, 298 \mathrm{~K}\right)$.

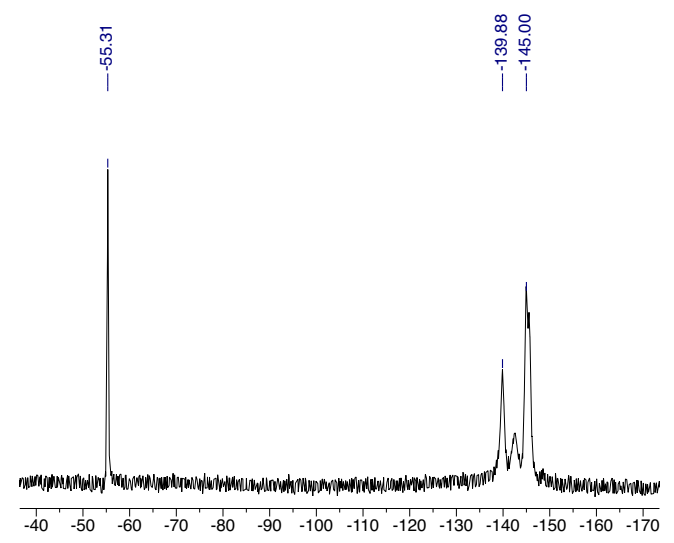

Figure S44. ${ }^{19} \mathrm{~F}$ NMR spectrum of $\mathbf{4}_{2} \subset \mathbf{1}\left(470 \mathrm{MHz}, \mathrm{D}_{2} \mathrm{O}, 298 \mathrm{~K}\right.$ ). 


\section{X-ray crystallography of complexes $\mathbf{2}_{2} \subset \mathbf{1}, \mathbf{3}_{2} \subset \mathbf{1}$, and $\mathbf{4}_{2} \subset \mathbf{1}$}

Table S1 lists the structural parameters of cage 1 (free and encapsulating BODIPYs 2-4). Crystals of inclusion complexes $\mathbf{2}_{2} \subset \mathbf{1}$ and $\mathbf{4}_{2} \subset \mathbf{1}$ contained a single type of species. Single crystals of $\mathbf{3}_{2} \subset \mathbf{1}$ contained two alternating inclusion complexes with slightly different conformations.

$\begin{array}{cccc}\text { Species } & \mathrm{Pd}_{\mathrm{ax}}-\mathrm{Pd}_{\mathrm{ax}} \text { distance } & \mathrm{Pd}_{\mathrm{eq}}-\mathrm{Pd}_{\mathrm{eq}} \text { distance } & \text { Angle at } \mathrm{Pd}_{\mathrm{ax}} \\ \mathbf{1} & 16.86 \AA & 18.23 \AA & 88.59^{\circ} \\ \mathbf{2}_{2} \subset \mathbf{1} & 18.44 \AA & 16.84 \AA & 76.38^{\circ} \\ \mathbf{3}_{2} \subset \mathbf{1} & 18.16 \AA / 18.59 \AA & 17.09 \AA / 16.70 \AA & 78.65^{\circ} / 75.37^{\circ} \\ \mathbf{4}_{2} \subset \mathbf{1} & 18.42 \AA & 16.91 \AA & 76.89^{\circ}\end{array}$

Table S1. Structural parameters for empty 1 and 1 encapsulating BODIPYs 2-4. $\mathrm{Pd}_{\mathrm{ax}}$ and $\mathrm{Pd}_{\mathrm{eq}}$ denote axial and equatorial palladium nodes, respectively; the $\mathrm{Pd}_{\mathrm{ax}}-\mathrm{Pd}_{\mathrm{ax}}$ distance is defined as the distance between two axial palladium nodes. The $\mathrm{Pd}_{\mathrm{eq}}-\mathrm{Pd}_{\mathrm{eq}}$ distance is defined as the average distance between two opposite equatorial palladium nodes of the cage. The angle at $\mathrm{Pd}_{\mathrm{ax}}$ is defined as angle between two TIm ligand planes at the axial position. All calculations of distances and angles were performed using Mercury 4.2.0 software.

Table S2 lists the structural parameters of BODIPYs 2-4 within the crystals of pure BODIPYs and the corresponding 2:1 inclusion complexes with cage $\mathbf{1}$.

$\begin{array}{cccc}\text { Species } & \text { Plane-to-plane distance } & \text { Center-to-center distance } & \text { Slip angle } \\ \mathbf{2}_{2} \subset \mathbf{1} & 3.58 \AA & 4.68 \AA & 65.35^{\circ} \\ \mathbf{2}_{\infty} & 3.52 \AA & 4.98 \AA & 65.05^{\circ} \\ \mathbf{3}_{2} \subset \mathbf{1} & 3.59 \AA / 3.62 \AA & 4.23 \AA / 4.34 \AA & 69.65^{\circ} / 73.45^{\circ} \\ \mathbf{3}_{\infty} & 3.57 \AA & 4.30 \AA & 71.87^{\circ} \\ \mathbf{4}_{2} \subset \mathbf{1} & 3.81 \AA & 4.83 \AA & 64.12^{\circ} \\ \mathbf{4}_{\infty} & 3.71 \AA & 6.49 \AA & 37.68^{\circ}\end{array}$

Table S2. Structural parameters for aggregated BODIPYs 2, 3, and $\mathbf{4}$ within single crystals and inside cage $\mathbf{1 .}$ For the calculation of plane-to-plane distances between two neighboring BODIPY units, individual planes were defined and calculated as surfaces based on the central $\mathrm{C}_{3} \mathrm{BN}_{2}$ ring of each BODIPY. For calculating the center-to-center distances, centroids within the same $\mathrm{C}_{3} \mathrm{BN}_{2}$ core units were calculated. The transition dipole moment of the $\mathrm{S}_{1} \leftarrow \mathrm{S}_{0}$ absorption is polarized along the long axis of the BODIPY chromophore. Thus, the slip angle, $\theta$, between the two dipole moments in a closely packed dimer was calculated as the inner angle of the triangle $\mathrm{C} 1$-centroid1-centroid2 (see the main text, Figure 1e, bottom). All calculations of distances, angles, centroids, and planes were performed using Mercury 4.2.0 software. 


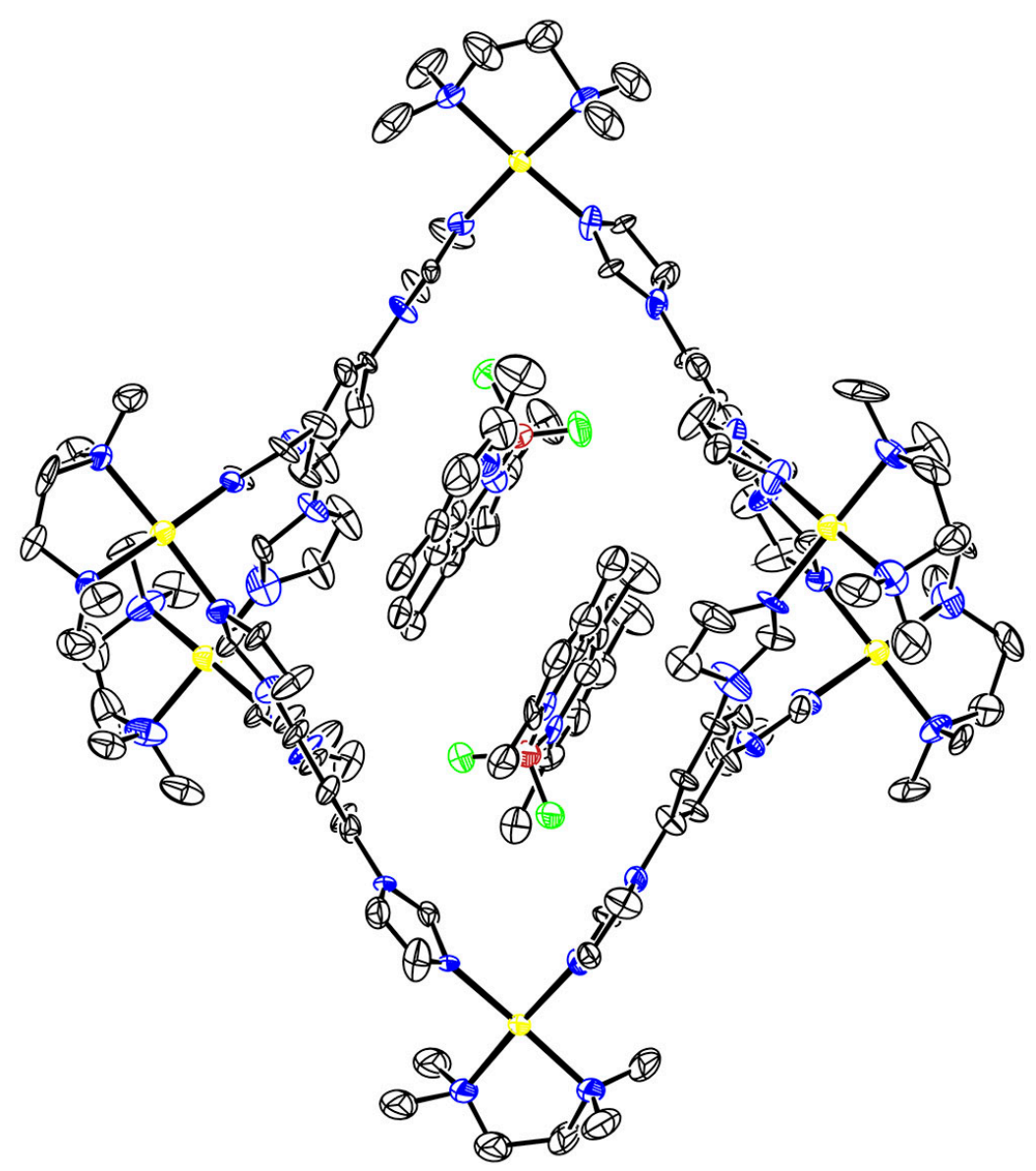

Figure S45. ORTEP representation of the X-ray structure of inclusion complex $\mathbf{2}_{2} \subset \mathbf{1}$ (thermal ellipsoids at a $50 \%$ probability level). Hydrogens, anions, and solvent molecules were eliminated for clarity. Pd, yellow; C gray; $\mathrm{N}$, blue; O, red; $\mathrm{B}$, brown; F, green. 


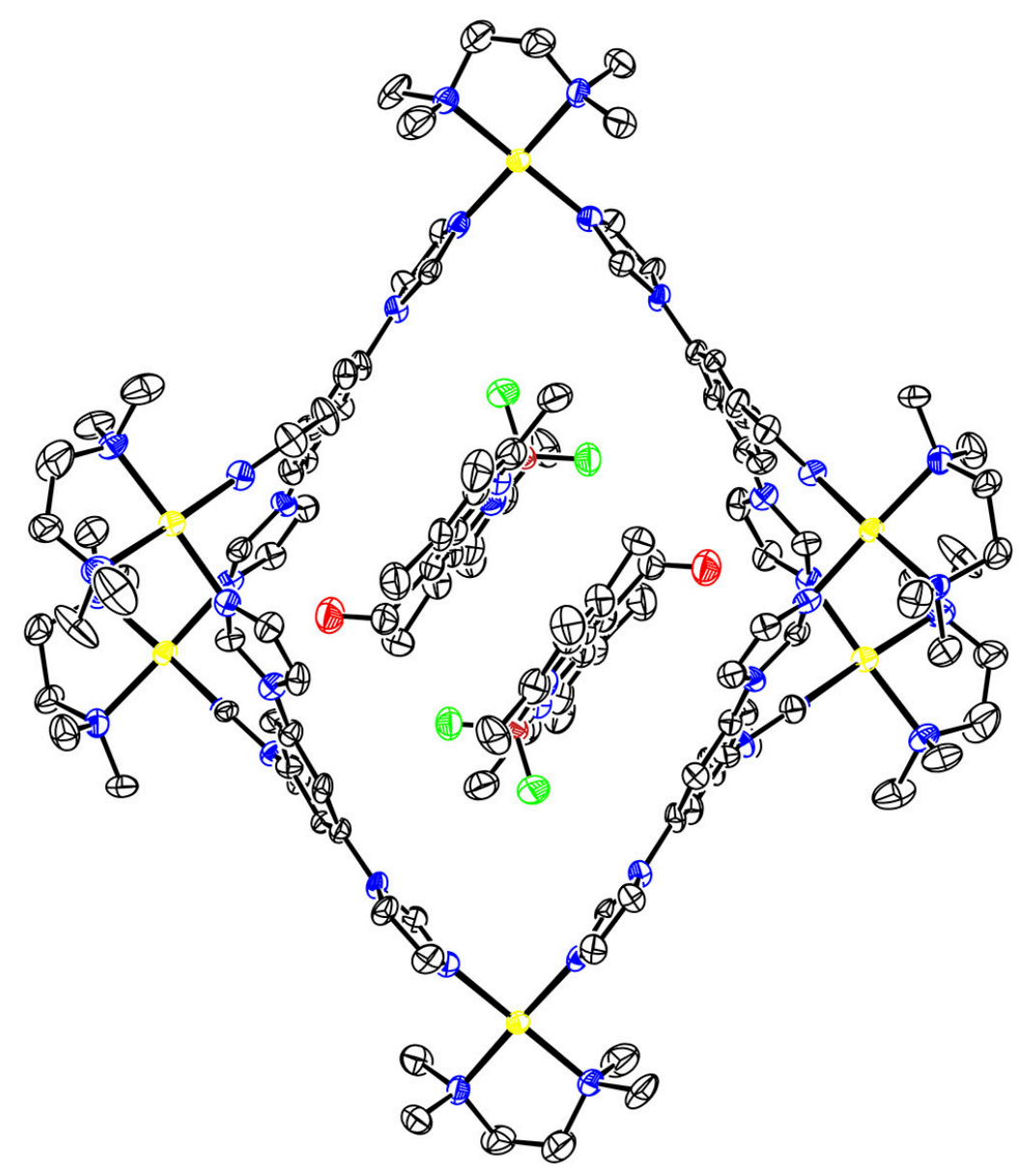

Figure S46. ORTEP representation of the X-ray structure of the first conformer of inclusion complex $\mathbf{3}_{2} \subset \mathbf{1}$ (thermal ellipsoids at a 50\% probability level). Hydrogens, anions, and solvent molecules were eliminated for clarity. Pd, yellow; C gray; N, blue; O, red; B, brown; F, green. 


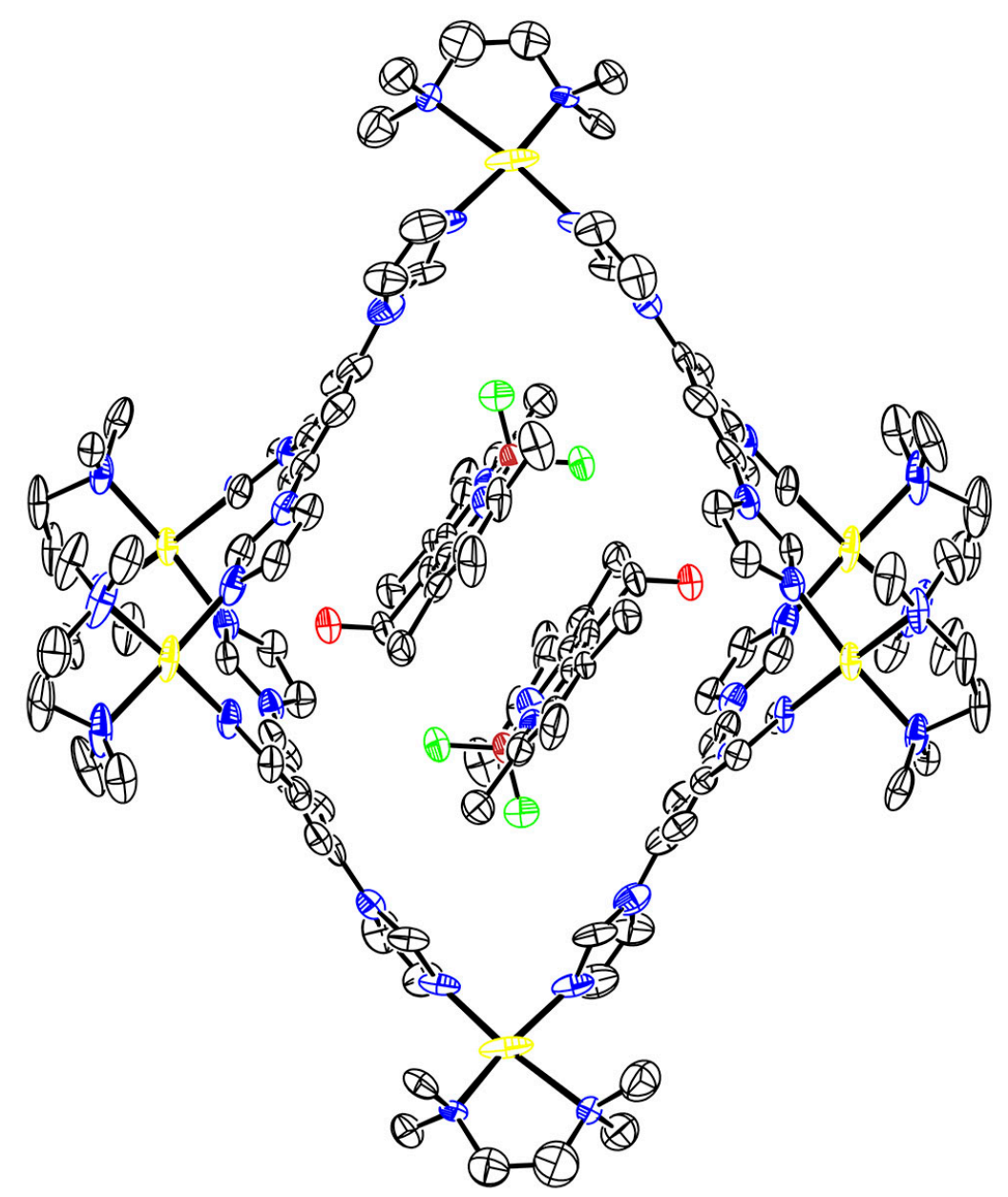

Figure S47. ORTEP representation of the X-ray structure of the second conformer of inclusion complex $\mathbf{3}_{2} \subset \mathbf{1}$ (thermal ellipsoids at a 50\% probability level). Hydrogens, anions, and solvent molecules were eliminated for clarity. Pd, yellow; C gray; N, blue; O, red; B, brown; F, green. 


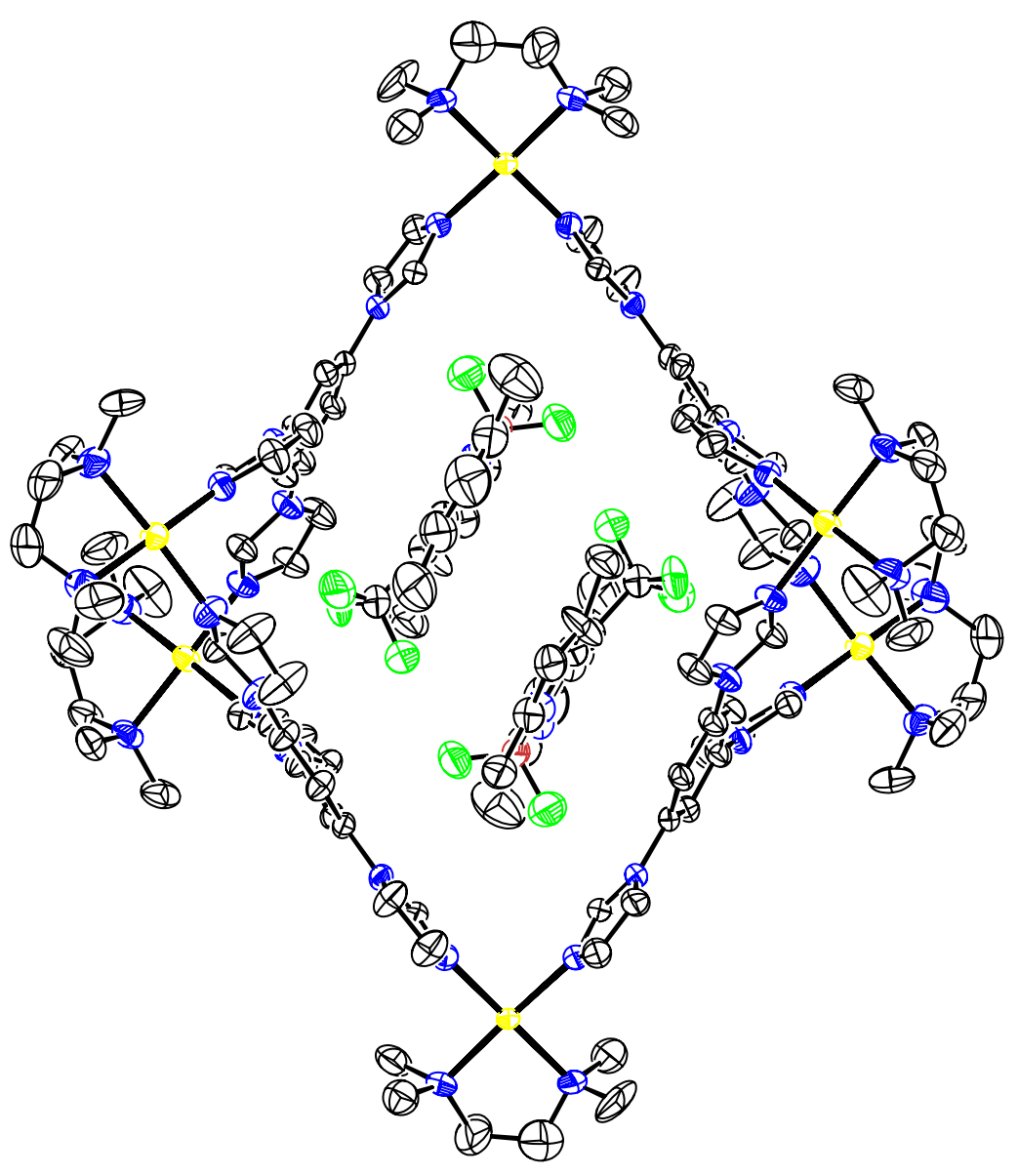

Figure S48. ORTEP representation of the X-ray structure of inclusion complex $\mathbf{4}_{2} \subset \mathbf{1}$ (thermal ellipsoids at a $50 \%$ probability level). Hydrogens, anions, and solvent molecules were eliminated for clarity. Pd, yellow; $\mathrm{C}$ gray; N, blue; B, brown; F, green.
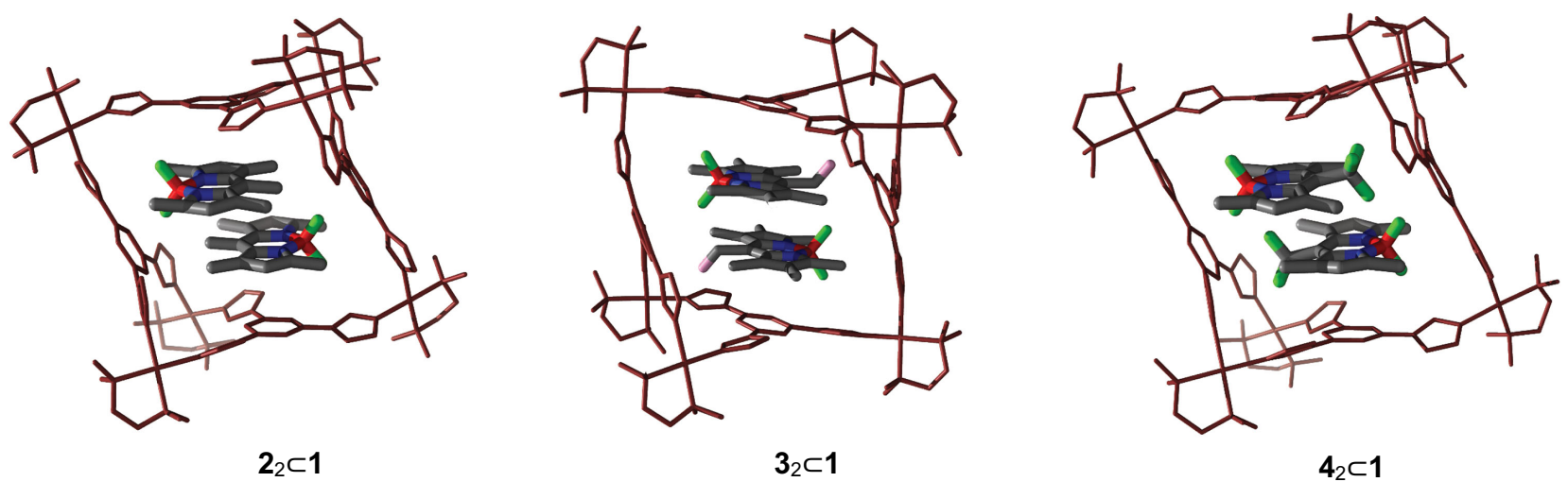

Figure S49. Comparison of the X-ray structures of $2_{2} \subset 1,3_{2} \subset 1$, and $4_{2} \subset 1$ (note that whereas the X-ray structures of $\mathbf{2}_{2} \subset \mathbf{1}$ and $\mathbf{4}_{2} \subset \mathbf{1}$ contained only one type of species, that of $\mathbf{3}_{2} \subset \mathbf{1}$ featured two slightly different conformations (in a 1:1 ratio). Only one of them in shown here; for the other conformation, see the CIF file). 


\section{Steady-state optical properties of $\mathbf{2}-\mathbf{4}$ and their inclusion complexes}

UV-vis absorption measurements were carried out on $0.04 \mathrm{mM}$ solutions of inclusion complexes (the concentration in terms of cage units) at ambient temperature. The solution of free cage $\mathbf{1}$ is practically transparent in the visible range, with an absorption onset at $\sim 380 \mathrm{~nm}$. BODIPY 2 dissolved in MeCN (note that 2-4 are all insoluble in water) exhibits optical behavior typical of classical BODIPY dyes, namely, a sharp absorption band in the visible range centered at $491 \mathrm{~nm}$ (corresponding to the $\mathrm{S}_{1} \leftarrow \mathrm{S}_{0}$ transition) with a blueshifted vibronic shoulder (see Figure S50A). A minor, broader absorption seen centered at $354 \mathrm{~nm}$ corresponds to the $\mathrm{S}_{2} \leftarrow \mathrm{S}_{0}$ transition. Fluorophore $\mathbf{2}$ exhibits an intense green fluorescence with the emission band mirroring the absorption band. The emission band is centered at $502 \mathrm{~nm}$, giving rise to a small Stokes shift (11 nm) characteristic of BODIPY dyes. BODIPY 3 has an intensive violet color, with a main absorbance band at 533 $\mathrm{nm}$. The fluorescence quantum yield of $\mathbf{3}$ is much lower than that of $\mathbf{2}$ due to quenching effects associated with the $\mathrm{CHO}$ group. The excitation spectrum recorded on a solution of $\mathbf{3}$ does not follow its absorption spectrum (excitation maximum $=517 \mathrm{~nm}$ ), with the emission band centered at the same wavelength as the absorption band $(533 \mathrm{~nm})$. We hypothesize that the excitation spectrum may originate from a minor yet highly emissive species, such as a rotamer or protonated 3. BODIPY 4 is red-purple with the main absorbance band at $548 \mathrm{~nm}$. As reported previously, ${ }^{4}$ it has a low fluorescence quantum yield, with a weak emission band at $595 \mathrm{~nm}$. Note that the absorption bands of $\mathbf{3}$ and $\mathbf{4}$ are significantly broader than for $\mathbf{2}$, which is likely due to the bulkiness of the substituents at the meso position $\left(\mathrm{CHO}\right.$ and $\left.\mathrm{CF}_{3}\right)$.

The absorption maxima of inclusion complexes $\mathbf{2}_{2} \subset \mathbf{1}, \mathbf{3}_{2} \subset \mathbf{1}$, and $\mathbf{4}_{2} \subset \mathbf{1}$ in water are blue-shifted with respect to the corresponding non-encapsulated dyes in $\mathrm{MeCN}$. For $\mathbf{2}_{2} \subset \mathbf{1}$, the main absorbance band is centered at 480 $\mathrm{nm}\left(\Delta_{\mathrm{abs}}=11 \mathrm{~nm}\right)$. The fluorescence is largely quenched and appears yellow, with the main emission band red-shifted to $544 \mathrm{~nm}$ (giving rise to a Stokes shift of $64 \mathrm{~nm}$; compare with $11 \mathrm{~nm}$ for free 2 in MeCN). The hypsochromic shift of the main absorption band and the decreased fluorescence quantum yield are well-known characteristics of the H-type dimers of chromophores stacked closely in an antiparallel fashion. These characteristics can be described by Kasha's model of exciton coupling in molecular dimers. ${ }^{5}$ For $\mathbf{3}_{2} \subset \mathbf{1}$, the absorbance band is centered at $520 \mathrm{~nm}\left(\Delta_{\text {abs }}=13 \mathrm{~nm}\right.$ ), with a largely quenched fluorescence (emission maximum $=539 \mathrm{~nm}$ ). The absorbance band of $\mathbf{4}_{2} \subset \mathbf{1}$ is centered at $523 \mathrm{~nm}$. The excitation spectra of $\mathbf{4}_{2} \subset \mathbf{1}$ does not follow the absorbance spectra, with the excitation maximum centered at $562 \mathrm{~nm}$.
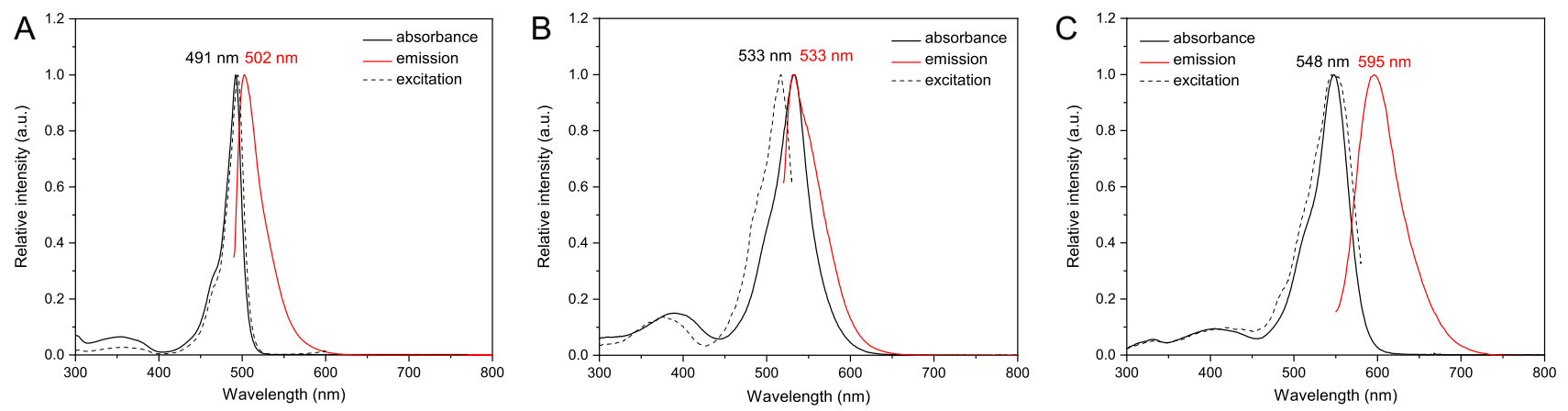

Figure S50. Normalized absorption, emission, and excitation spectra of (A) 2 ( $\left.\lambda_{\mathrm{exc}}=460 \mathrm{~nm}\right),(\mathrm{B}) 3\left(\lambda_{\mathrm{exc}}=500\right.$ $\mathrm{nm})$, and $(\mathrm{C}) 4\left(\lambda_{\mathrm{exc}}=530 \mathrm{~nm}\right)$, all in MeCN. The wavelengths of maximum absorption and emission are denoted in black and red, respectively. The wavelengths of maximum excitation are very similar to those of the absorption spectra, except for (B), where $\lambda_{\max }=517 \mathrm{~nm}$ (note that the fluorescence quantum yield of 3 is low; the excitation and emission spectra could originate from a highly emissive rotamer or another species, such as protonated $\mathbf{3}$ ). 

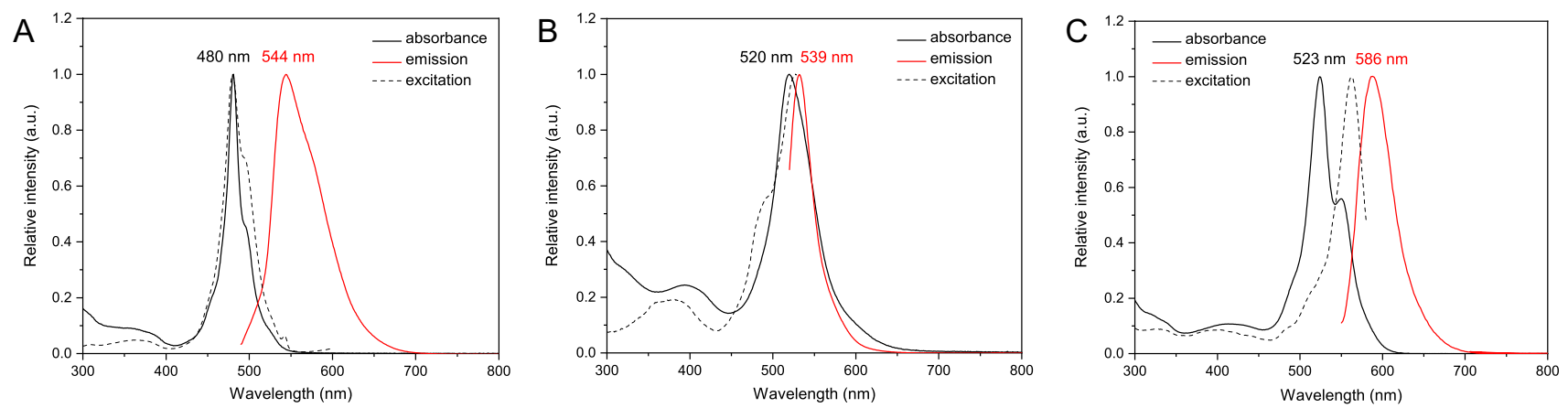

Figure S51. Normalized absorption, emission, and excitation spectra of (A) $\mathbf{2}_{2} \subset \mathbf{1}\left(\lambda_{\mathrm{exc}}=460 \mathrm{~nm}\right),(\mathrm{B}) \mathbf{3}_{2} \subset \mathbf{1}$ $\left(\lambda_{\mathrm{exc}}=500 \mathrm{~nm}\right)$, and $(\mathrm{C}) \mathbf{4}_{2} \subset \mathbf{1}\left(\lambda_{\mathrm{exc}}=530 \mathrm{~nm}\right)$, all in $\mathrm{H}_{2} \mathrm{O}$. The wavelengths of maximum absorption and emission are denoted in black and red, respectively. The wavelengths of maximum excitation are very similar to those of the absorption spectra, except for (C), where $\lambda_{\max }=562 \mathrm{~nm}$.

Addition of $\mathrm{MeCN}$ to an aqueous solution of $\mathbf{2}_{2} \subset \mathbf{1}$ instantly results in a significant (fluorescence) color change of the solution, as shown below:

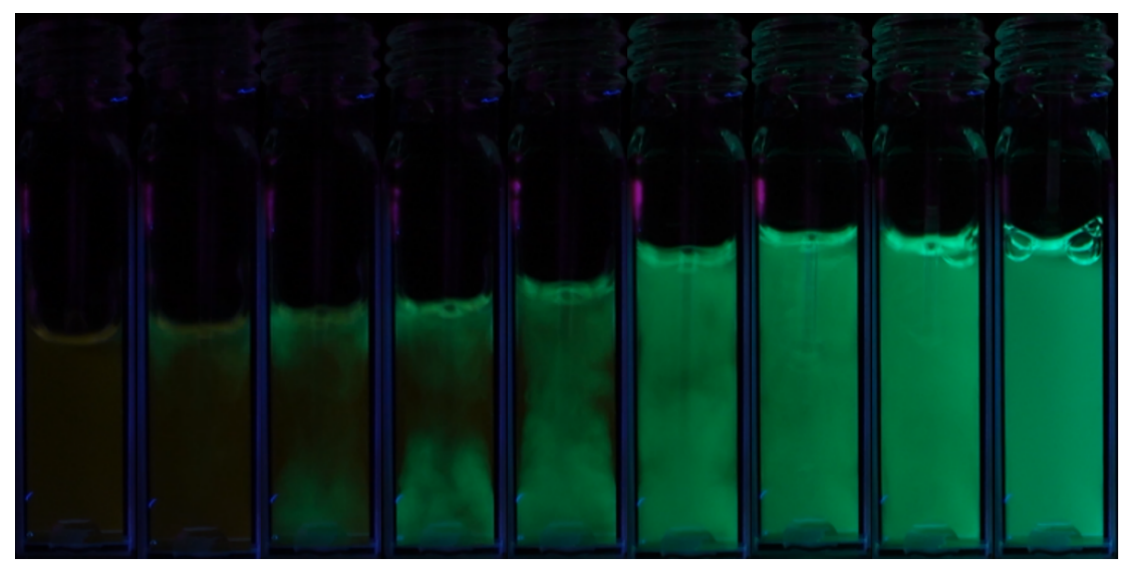

Figure S52. The instantaneous emission color change of an aqueous solution of $\mathbf{2}_{2} \subset \mathbf{1}$ upon the addition of MeCN $\left(\lambda_{\mathrm{exc}}=365 \mathrm{~nm}\right)$.

To investigate this process in more detail, we followed the process by UV-vis absorption and fluorescence spectroscopies. We tested several organic solvents, in which BODIPYs $\mathbf{2 - \mathbf { 4 }}$ are readily soluble (MeCN, acetone, DMF, 1,4-dioxane, DMSO, and MeOH). In a typical experiment, a $0.04 \mathrm{mM}$ solution of $\mathbf{2}_{2} \subset \mathbf{1}$ in $2 \mathrm{~mL}$ of water (the concentration in terms of cage units) was treated with $100 \mu \mathrm{L}$ aliquots of an organic solvent. After the addition of each aliquot, the solution was thoroughly mixed and the spectrum was recorded. The aliquots were added until no further changes in the spectra (other than those due to dilution) were observed. We verified that in all the experiments, all the species $\left(\mathbf{1}, \mathbf{2}\right.$, and $\left.\mathbf{2}_{2} \subset \mathbf{1}\right)$ remained dissolved in solution.

The release of $\mathbf{2}$ from $\mathbf{2}_{2} \subset \mathbf{1}$ upon the addition of MeCN was accompanied by the appearance of a sharp, intense absorption band, characteristic of monomeric BODIPYs in solution (see Figure S53A). In addition, fluorescence spectroscopy showed a rapid increase in the emission intensity (Figure S53C).

Concentration-corrected spectra show that the absorption coefficient of $\mathbf{2}$ within $\mathbf{1}, \varepsilon_{2}^{\prime}$, is substantially smaller than that of free 2 in solution ( $\varepsilon_{2}$; specifically, we found that $\varepsilon_{2}{ }^{\prime} \approx 0.45 \varepsilon_{2}$ ). In other words, the release of 2 from the cage leads not only to a bathochromic shift but also to a substantial increase in the absorption intensity. Provided that the final solution contains only monomeric 2 in solution (i.e., no encapsulated 2 ), we can calculate, based on the molar absorption coefficient of $2\left(\sim 80,000 \mathrm{~L} \mathrm{~mol}^{-1} \mathrm{~cm}^{-1}\right),{ }^{4}$ the percentage of $\mathbf{1}$ 
encapsulating the noncovalent dimer of 2 . The result of this calculation $(\sim 50 \%)$ is in a very good agreement with the encapsulation efficiency determined by ${ }^{1} \mathrm{H}$ NMR.

Next, we investigated the release of $\mathbf{2}$ from $\mathbf{2}_{2} \subset \mathbf{1}$ using other organic solvents. As Figure S53B shows, the release commences only after a certain volume percentage of a polar organic solvent has been added. We found that $\mathrm{MeCN}$, dioxane, acetone, and DMF were similarly efficient in releasing the dye from the cage. However, higher volume fractions of DMSO and $\mathrm{MeOH}$ had to be added to reach the same level of release. In all cases, the final absorption spectra were identical to those of free $\mathbf{2}$ in the corresponding organic solvent, indicating that 2 was released from the cage in a quantitative fashion.

The same experiments were repeated with BODIPY $\mathbf{4}$ and inclusion complex $\mathbf{4}_{2} \subset \mathbf{1}$ (Figures S53D-F). The results were analogous, except that, in contrast to BODIPY $\mathbf{2}$, the fluorescence emission of $\mathbf{4}$ is higher inside the cage than in organic solution, resulting in decreased fluorescence emission upon the addition of an organic solvent (see Figure S53F). Given the molar extinction coefficient of free $4, \varepsilon 4^{\prime} \approx 45,000 \mathrm{~L} \mathrm{~mol}^{-1} \mathrm{~cm}^{-1},{ }^{4}$ the percentage of filled 1 at the onset of the experiment was determined as $62 \%$.
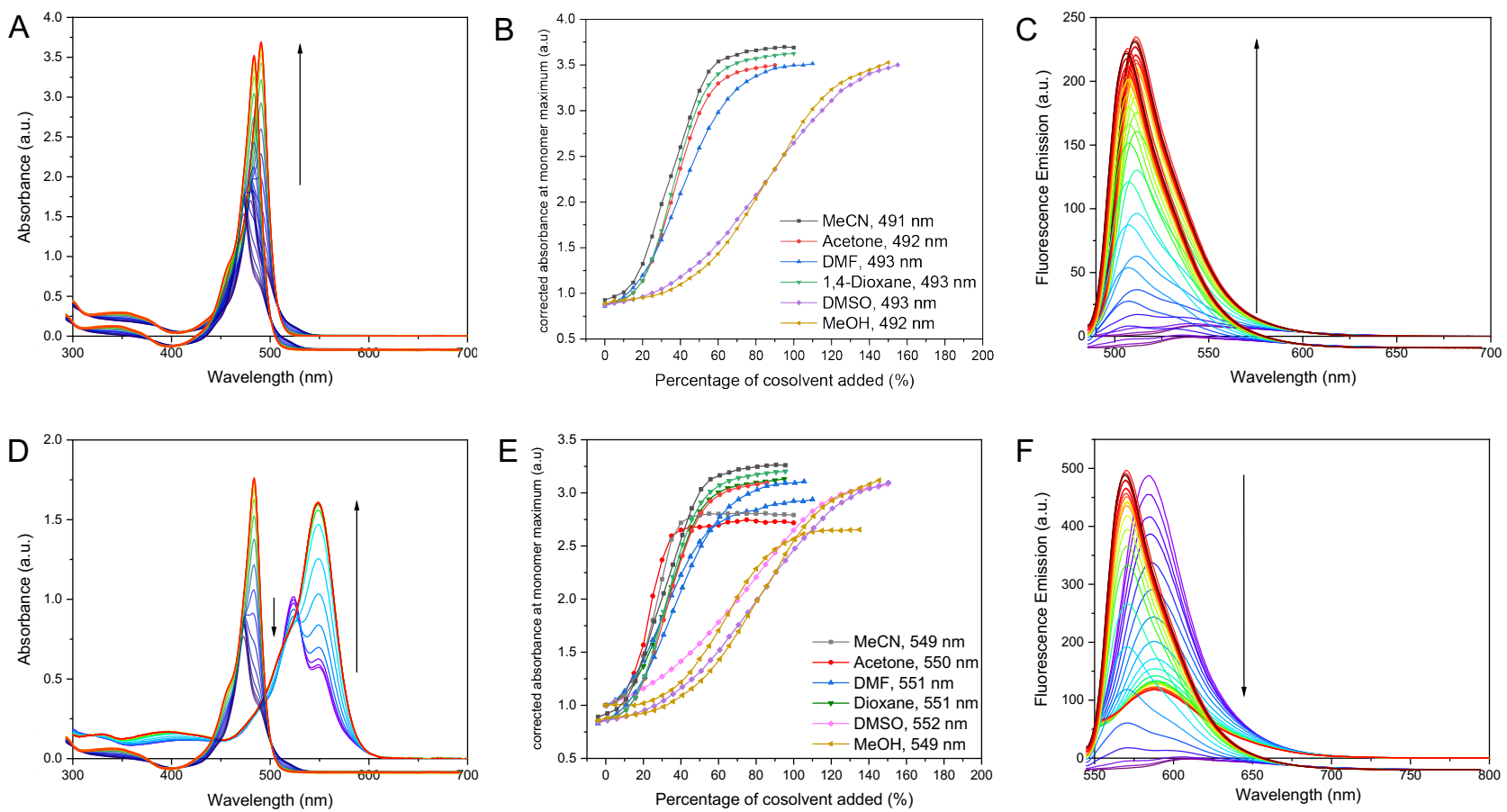

Figure S53. (A) Changes in the absorption spectrum of $\mathbf{2}_{2} \subset \mathbf{1}$ in $\mathrm{H}_{2} \mathrm{O}$ upon the gradual addition of MeCN (concentration-corrected). We found that the extinction coefficient of $\mathbf{2}$ within $\mathbf{1}, \varepsilon\left(\mathbf{2}_{2} \subset \mathbf{1}\right)$, amounts to roughly $0.45 \times \varepsilon(2)$. (B) Plots of concentration-corrected absorbance (at the wavelength of maximum absorption of free 2 in a given solvent) as a function of the volume of organic solvent added. (C) Changes in the emission spectrum $\left(\lambda_{\mathrm{exc}}=460 \mathrm{~nm}\right.$ ) of $\mathbf{2}_{2} \subset \mathbf{1}$ in $\mathrm{H}_{2} \mathrm{O}$ upon the gradual addition of $\mathrm{MeCN}$ (concentration-corrected). (D) Changes in the absorption spectrum of $\mathbf{4}_{2} \mathrm{Cl}$ in $\mathrm{H}_{2} \mathrm{O}$ upon the gradual addition of $\mathrm{MeCN}$ (concentrationcorrected). (E) Plots of concentration-corrected absorbance (at the wavelength of the maximum absorption of free 4 in a given solvent) as a function of the volume of organic solvent added. (F) Changes in the emission spectrum $\left(\lambda_{\mathrm{exc}}=530 \mathrm{~nm}\right.$ ) of $\mathbf{4}_{2} \subset \mathbf{1}$ in $\mathrm{H}_{2} \mathrm{O}$ upon the gradual addition of $\mathrm{MeCN}$ (concentration-corrected). 
Samples for solid-state UV-vis absorption spectra were prepared by dropcasting MeCN solutions of BODIPYs 2-4 and aqueous solutions of inclusion complexes $\mathbf{2}_{2} \subset \mathbf{1}, \mathbf{3}_{2} \subset \mathbf{1}$, and $\mathbf{4}_{2} \subset \mathbf{1}$ on glass slides, followed by solvent evaporation. For solution-state spectra of free 2, 3, and 4, MeCN was used as the solvent. Samples of aggregates of $\mathbf{2}, \mathbf{3}$, and $\mathbf{4}$ were prepared by diluting $\mathrm{MeCN}$ solutions of the compounds with 49 volumes of $\mathrm{H}_{2} \mathrm{O}$; the spectra were recorded $\sim 30 \mathrm{~s}$ after the addition of $\mathrm{H}_{2} \mathrm{O}$. For solid-state spectra of $\mathbf{2}_{2} \subset \mathbf{1}$ and $\mathbf{4}_{2} \subset \mathbf{1}$, note that the samples contain residual unfilled cages ( $\sim 1$ equiv of free $\mathbf{1}$ for $\mathbf{2}_{2} \subset \mathbf{1}$ and $\sim 0.6$ equiv of free $\mathbf{1}$ for $\mathbf{4}_{2} \subset \mathbf{1}$ ).
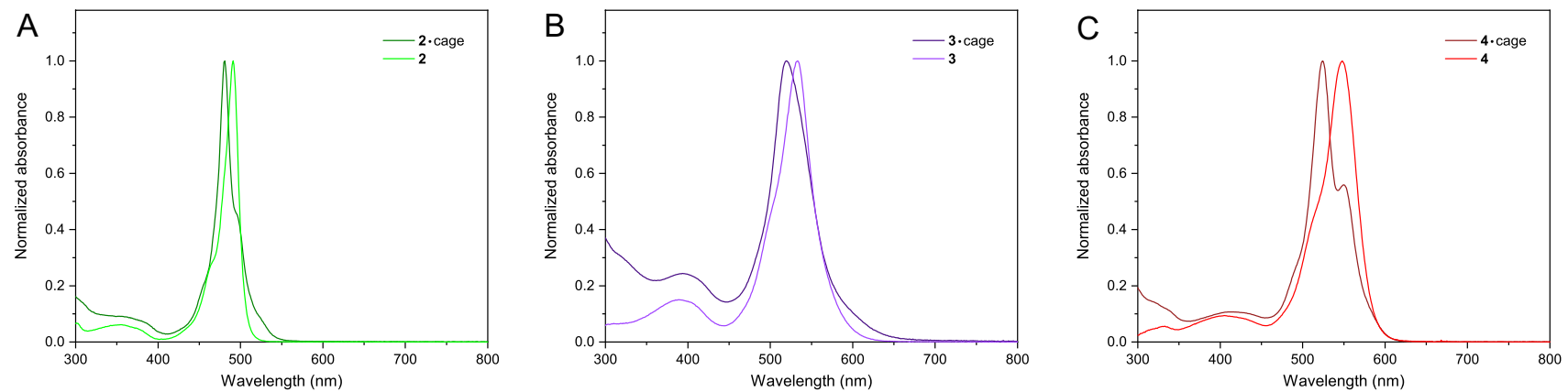

Figure S54. (A) Comparison of normalized solution UV-vis absorption spectra of $\mathbf{2}(\mathrm{MeCN})$ and $\mathbf{2}_{2} \subset \mathbf{1}\left(\mathrm{H}_{2} \mathrm{O}\right)$. (B) Comparison of normalized solution UV-vis absorption spectra of $\mathbf{3}(\mathrm{MeCN})$ and $\mathbf{3}_{2} \subset \mathbf{1}\left(\mathrm{H}_{2} \mathrm{O}\right)$. (C) Comparison of normalized solution UV-vis absorption spectra of $\mathbf{4}(\mathrm{MeCN})$ and $\mathbf{4}_{2} \subset \mathbf{1}\left(\mathrm{H}_{2} \mathrm{O}\right)$.
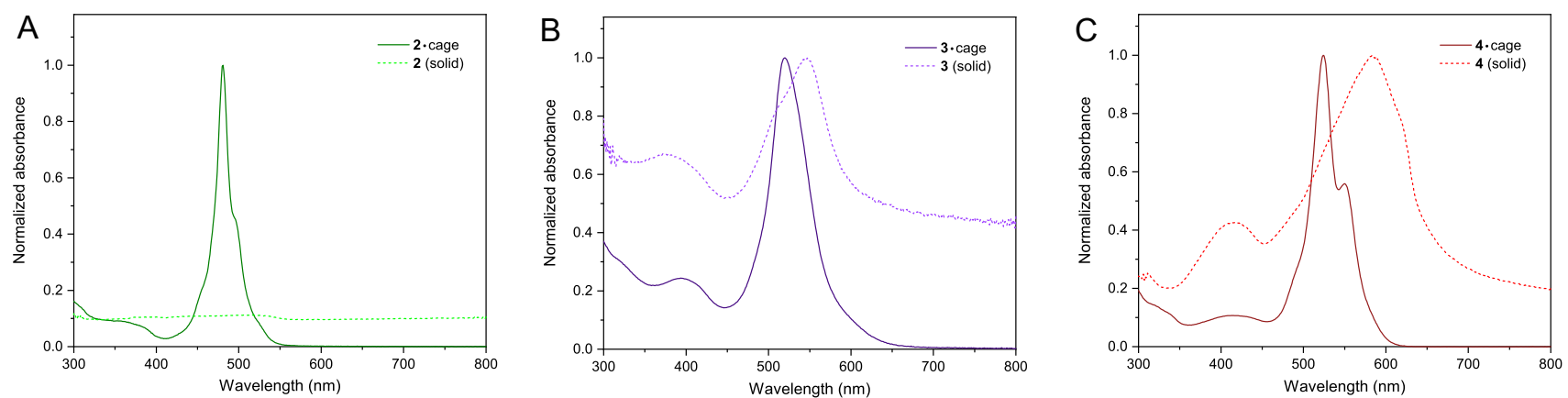

Figure S55. (A) Comparison of normalized solution $(\mathrm{MeCN})$ and solid-state UV-vis absorption spectra of 2. (Note that due to the formation of large crystals as opposed to continuous films, the solid-state spectrum of 2 is featureless and has not been normalized.) (B) Comparison of normalized solution $(\mathrm{MeCN})$ and solid-state UV-vis absorption spectra of 3. (C) Comparison of normalized solution (MeCN) and solid-state UV-vis absorption spectra of $\mathbf{4}$.
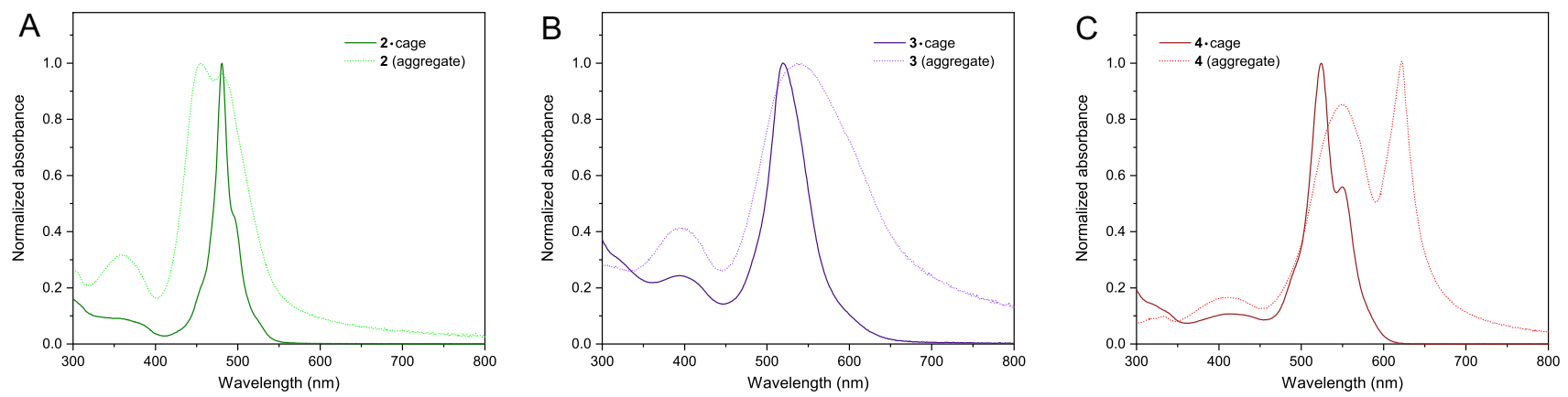

Figure S56. (A) Comparison of normalized UV-vis absorption spectra of $\mathbf{2}_{2} \subset \mathbf{1}\left(\mathrm{H}_{2} \mathrm{O}\right)$ and aggregated 2 $\left(\mathrm{H}_{2} \mathrm{O} / \mathrm{MeCN}, v / v=49: 1\right)$. (B) Comparison of normalized UV-vis absorption spectra of $\mathbf{3}_{2} \subset \mathbf{1}\left(\mathrm{H}_{2} \mathrm{O}\right)$ and aggregated $3\left(\mathrm{H}_{2} \mathrm{O} / \mathrm{MeCN}, v / v=49: 1\right)$. (C) Comparison of normalized UV-vis absorption spectra of $\mathbf{4}_{2} \subset \mathbf{1}$ $\left(\mathrm{H}_{2} \mathrm{O}\right)$ and aggregated $4\left(\mathrm{H}_{2} \mathrm{O} / \mathrm{MeCN}, v / v=49: 1\right)$. 

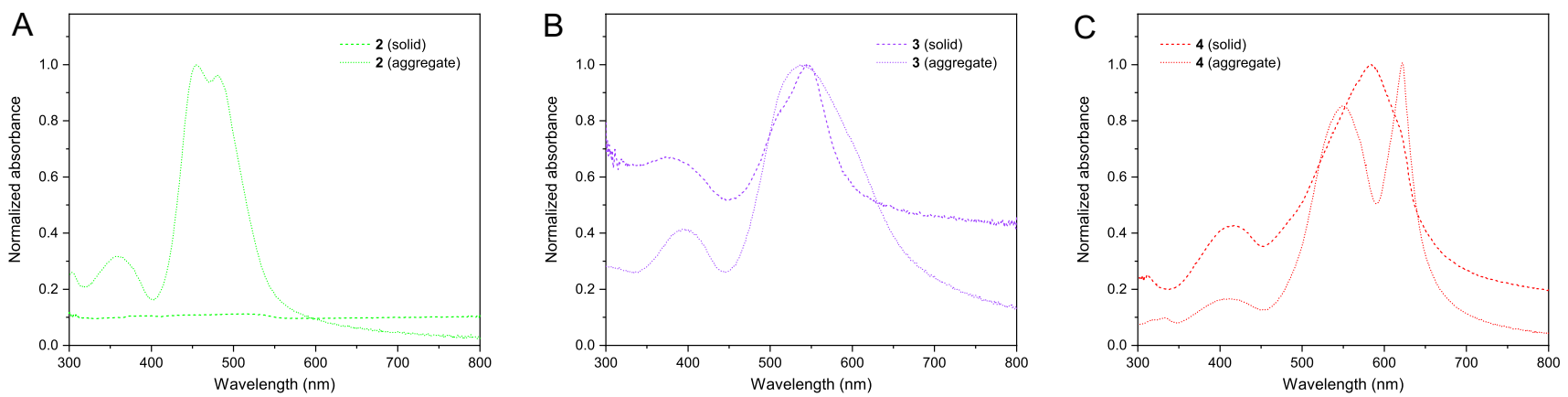

Figure S57. (A) Comparison of the solid-state UV-vis absorption spectrum of $\mathbf{2}$ and the solution spectrum of aggregated $2\left(\mathrm{H}_{2} \mathrm{O} / \mathrm{MeCN}, v / v=49: 1\right.$; normalized). (Note that due to the formation of large crystals as opposed to continuous films, the solid-state spectrum of $\mathbf{2}$ is featureless and has not been normalized.) (B) Comparison of the solid-state UV-vis absorption spectrum of $\mathbf{3}$ and the solution spectrum of aggregated $\mathbf{3}\left(\mathrm{H}_{2} \mathrm{O} / \mathrm{MeCN}, v / v\right.$ $=49: 1$ ) (both normalized). (D) Comparison of the solid-state UV-vis absorption spectrum of 3 and the solution spectrum of aggregated $\mathbf{3}\left(\mathrm{H}_{2} \mathrm{O} / \mathrm{MeCN}, v / v=49: 1\right)$ (both normalized).
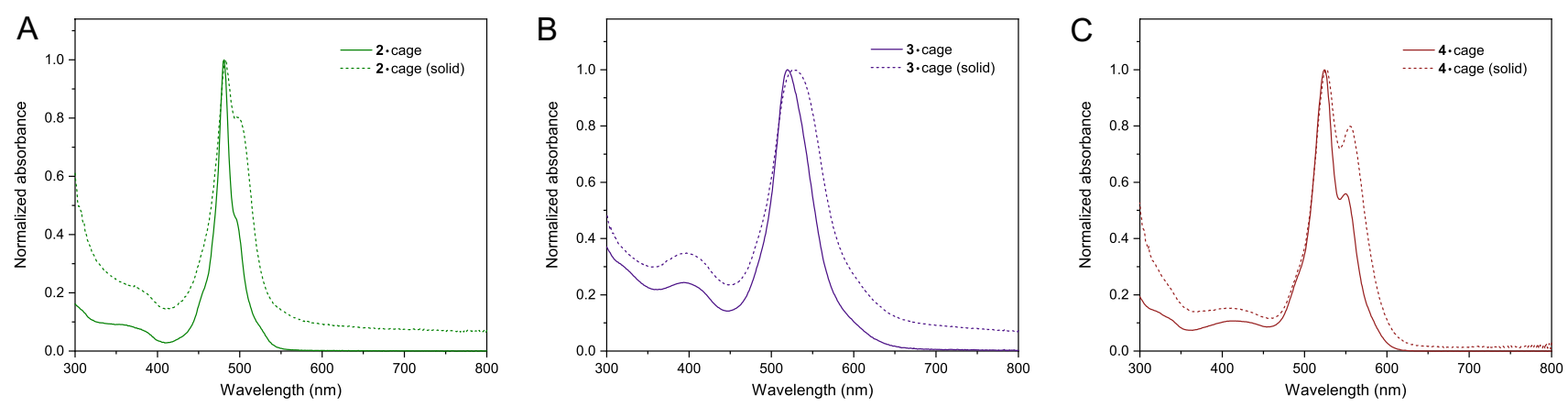

Figure S58. (A) Comparison of normalized solution $\left(\mathrm{H}_{2} \mathrm{O}\right)$ and solid-state $\mathrm{UV}-$ vis absorption spectra of $\mathbf{2}_{2} \subset \mathbf{1}$. (B) Comparison of normalized solution $\left(\mathrm{H}_{2} \mathrm{O}\right)$ and solid-state $\mathrm{UV}-\mathrm{vis}$ absorption spectra of $\mathbf{3}_{2} \subset \mathbf{1}$. (C) Comparison of normalized solution $\left(\mathrm{H}_{2} \mathrm{O}\right)$ and solid-state $\mathrm{UV}$-vis absorption spectra of $\mathbf{4}_{2} \subset \mathbf{1}$.
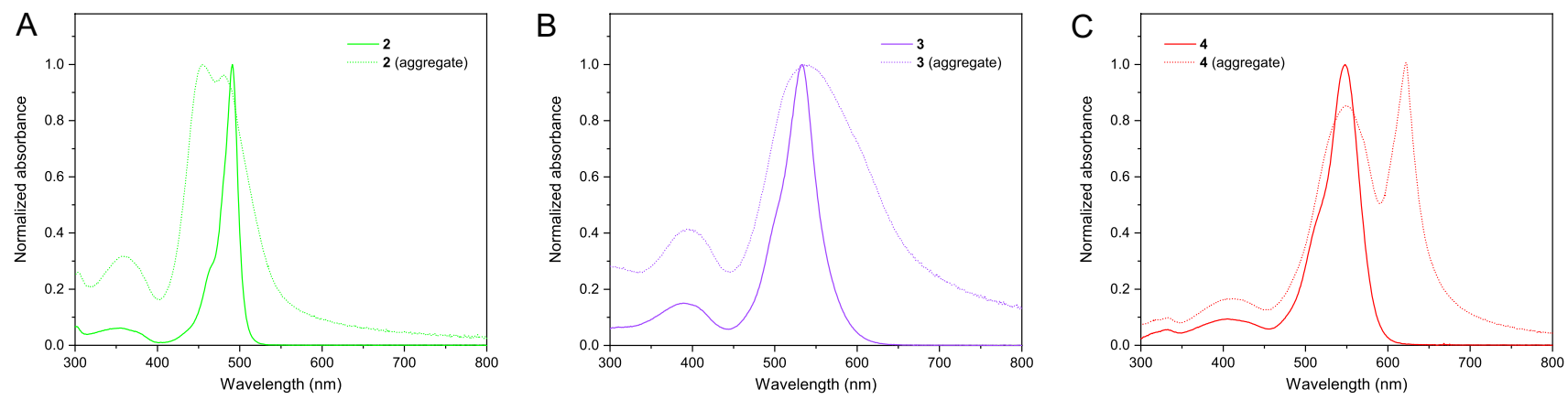

Figure S59. (A) Comparison of normalized UV-vis absorption spectra of free $(\mathrm{MeCN})$ and aggregated $\left(\mathrm{H}_{2} \mathrm{O} / \mathrm{MeCN}, v / v=49: 1\right)$ 2. (B) Comparison of normalized UV-vis absorption spectra of free (MeCN) and aggregated $\left(\mathrm{H}_{2} \mathrm{O} / \mathrm{MeCN}, v / v=49: 1\right) 3$. (C) Comparison of normalized UV-vis absorption spectra of free $(\mathrm{MeCN})$ and aggregated $\left(\mathrm{H}_{2} \mathrm{O} / \mathrm{MeCN}, v / v=49: 1\right) 4$. 

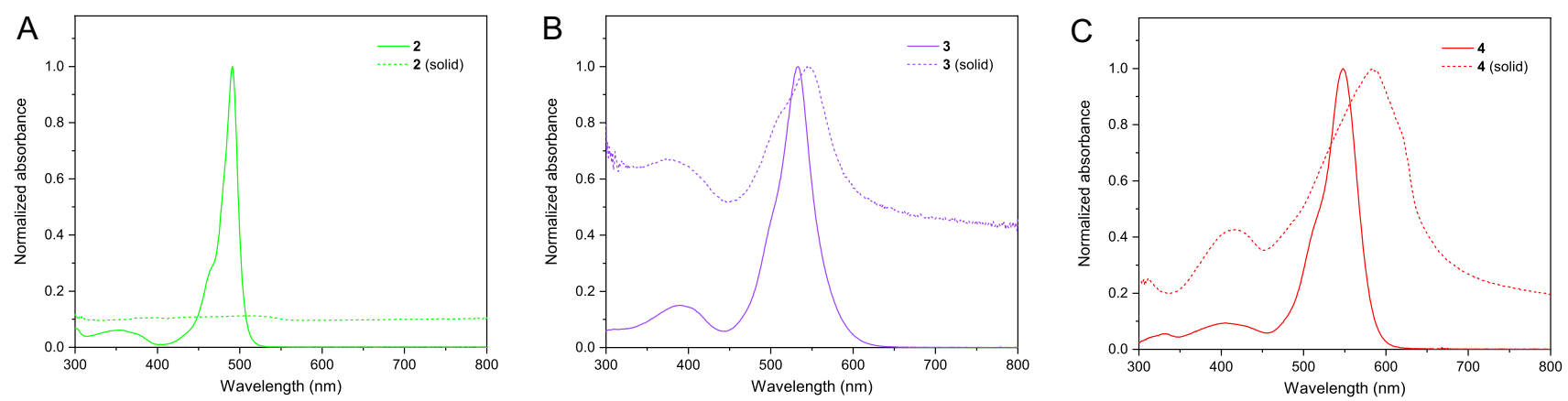

Figure S60. (A) Comparison of solution (MeCN; normalized) and solid-state UV-vis absorption spectra of 2. (Note that due to the formation of large crystals as opposed to continuous films, the solid-state spectrum of $\mathbf{2}$ is featureless and has not been normalized.) (B) Comparison of normalized solution ( $\mathrm{MeCN}$ ) and solid-state UV-vis absorption spectra of 3. (C) Comparison of normalized solution (MeCN) and solid-state UV-vis absorption spectra of 4 .
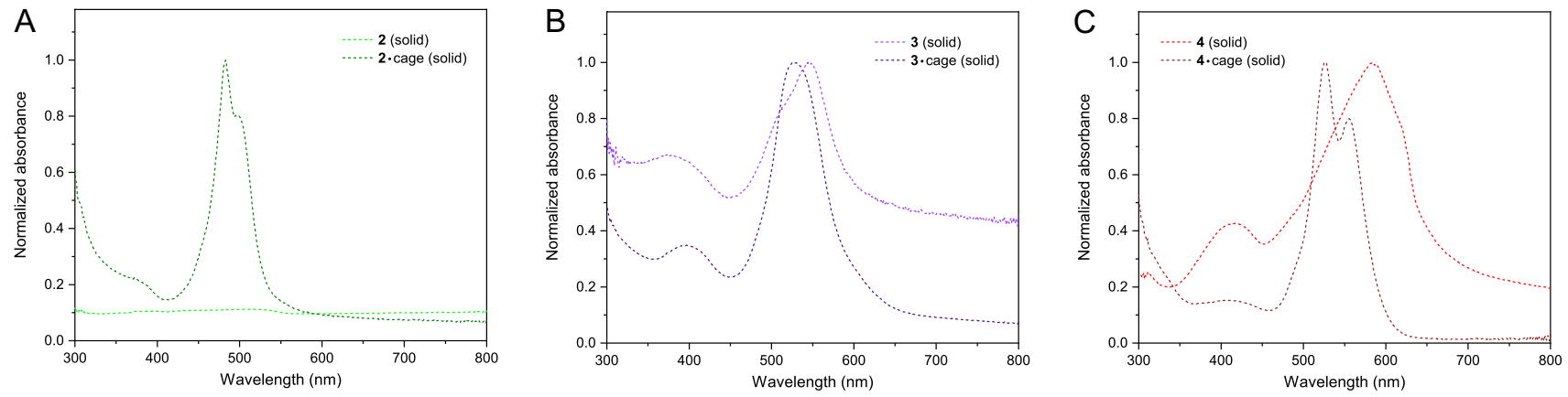

Figure S61. (A) Comparison of solid-state UV-vis absorption spectra of $\mathbf{2}_{2} \subset \mathbf{1}$ (normalized) and 2. (Note that due to the formation of large crystals as opposed to continuous films, the solid-state spectrum of $\mathbf{2}$ is featureless and has not been normalized.) (B) Comparison of normalized solid-state UV-vis absorption spectra of $\mathbf{3}$ and $\mathbf{3}_{2} \subset \mathbf{1}$. (C) Comparison of normalized solid-state UV-vis absorption spectra of $\mathbf{4}$ and $\mathbf{4}_{2} \subset \mathbf{1}$.

\section{Time-resolved optical properties of $\mathbf{2}-\mathbf{4}$ and their inclusion complexes}

For $\mathbf{2}$ and $\mathbf{2}_{2} \subset \mathbf{1}$, femtosecond transient absorption measurements were carried out on a system based on a mode-locked Ti:sapphire oscillator (Spectra Physics Mai Tai SP). The oscillator produced a train of $<120$ fs pulses (bandwidth $\sim 12 \mathrm{~nm}$ FWHM) with a peak wavelength at $800 \mathrm{~nm}$, typically of $900 \mathrm{~mW}$, corresponding to $\sim 10 \mathrm{~nJ}$ per pulse. The weak oscillator pulses were amplified by a chirped-pulse regenerative amplifier (CPA, Spectra Physics Spitfire Ace). The pulses were first stretched to several picoseconds, then regeneratively amplified in a Ti:sapphire cavity, pumped by a pulsed Nd:YLF laser (Spectra Physics Empower 45) operating at $1 \mathrm{kHz}$. After the pulse was amplified and recompressed, its energy was about $5 \mathrm{~mJ}$ in a train of $1,000 \mathrm{~Hz}$ pulses. An independent pump pulse was obtained by pumping an optical parametric amplifier (Spectra Physics OPA-800CF) that produces 120-fs pulses tunable from $300 \mathrm{~nm}$ to $3 \mu \mathrm{m}$. One Watt of light amplified on Spitfire was used; the output power of the OPA varied between a few $\mu \mathrm{J}$ and tens of $\mu \mathrm{J}$ (depending on the chosen wavelength) at 1,000 Hz. In the reported experiments, the pump was turned to $440 \mathrm{~nm}$ and the optical densities of the samples in $1 \mathrm{~mm}$ and $2 \mathrm{~mm}$ optical path length cuvettes were kept between 0.2 and 0.4 at the excitation wavelength. Spectral corrections and analyses were performed using SURFACE XPLORER Pro (Ultrafast Systems) and Origin 9.1 (OriginLab) software. The nanosecond fluorescence was measured using a LP920 flash photolysis system (Edinburgh Instruments, UK). The system was pumped by a Nd:YAG third harmonic (355 nm) driving an OPO (Spectra Physics, USA, Quanta-Ray Lab series, and GWU Lasertechnik, Germany, VersaScan-355 midband). The spectra were collected on an intensified CCD camera (i-Star, Andor, UK), where the delay was determined by gating the image intensifier. 

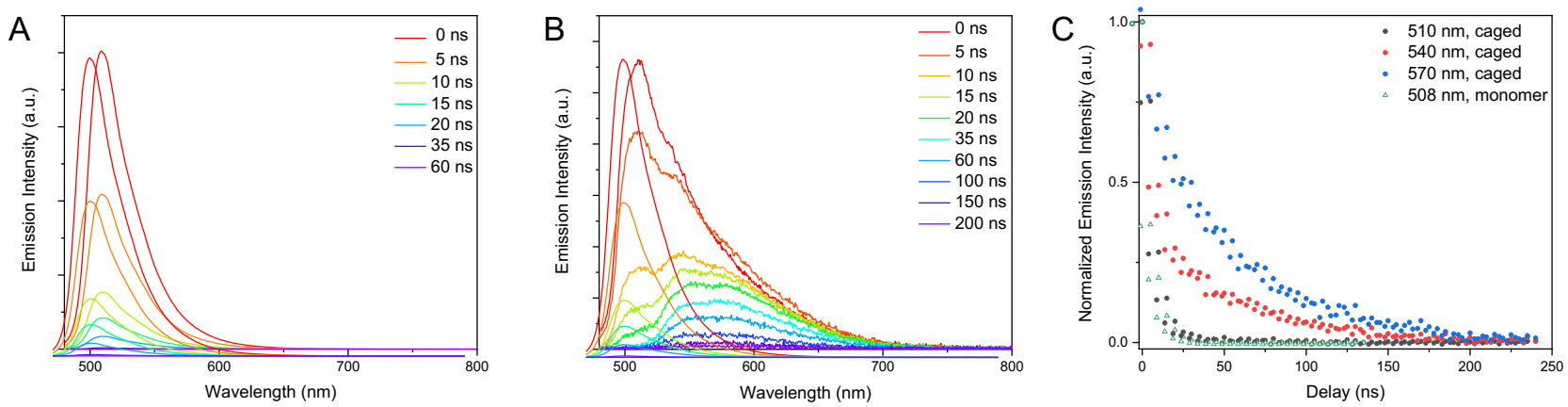

Figure S62. (A) Time-resolved fluorescence spectra of $\mathbf{2}$ dissolved in MeCN. (B) Time-resolved fluorescence spectra of $\mathbf{2}_{2} \subset \mathbf{1}$ in $\mathrm{H}_{2} \mathrm{O}$ (replotted from Figure $2 \mathrm{~b}$ in the main text). (C) Decay of fluorescence of $\mathbf{2}$ and $\mathbf{2}_{2} \subset \mathbf{1}$ at three different wavelengths.

For $\mathbf{3}, \mathbf{3}_{2} \subset \mathbf{1}, \mathbf{4}$, and $\mathbf{4}_{2} \subset \mathbf{1}$, the samples were excited by a frequency-tripled $\mathrm{Nd}$ :YAG Q-switched laser, pumping an optical parametric oscillator (Ekspla NT342/C/3/UVE) with a pulse duration of $5 \mathrm{~ns}$ and a repetition rate of $10 \mathrm{~Hz}$. The fluorescence was collected in the direction orthogonal to the laser incident beam using a $20 \times 0.4 \mathrm{NA}$ objective and spectrally filtered using a long pass filter onto a monochromator (Acton SpectraPro2150i), coupled to a photomultiplier (PMT) tube (Hamamatsu R10699). Transient emission measurements were recorded by a $600 \mathrm{MHz}$ digital oscilloscope (LeCroy Wavesurfer 62Xs). Emission spectra were plotted by integrating over transient emission curve at each wavelength. Excitation pulse energy was measured by a pyroelectric sensor (PE9-C, Ophir Optronics). The same setup was used to acquire time-resolved spectra of 2 and $\mathbf{2}_{2} \subset \mathbf{1}$, and results similar to those in Figure S62 were obtained.
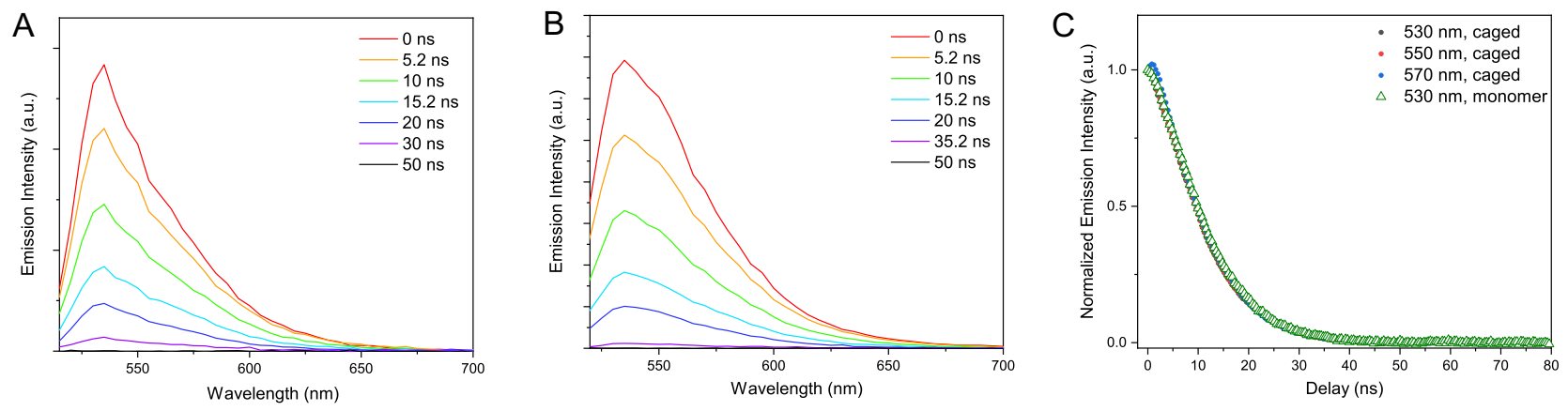

Figure S63. (A) Time-resolved fluorescence spectra of $\mathbf{3}$ dissolved in MeCN. (B) Time-resolved fluorescence spectra of $\mathbf{3}_{2} \subset \mathbf{1}$ in $\mathrm{H}_{2} \mathrm{O}$. (C) Decay of fluorescence of $\mathbf{3}$ and $\mathbf{3}_{2} \subset \mathbf{1}$ at three different wavelengths.
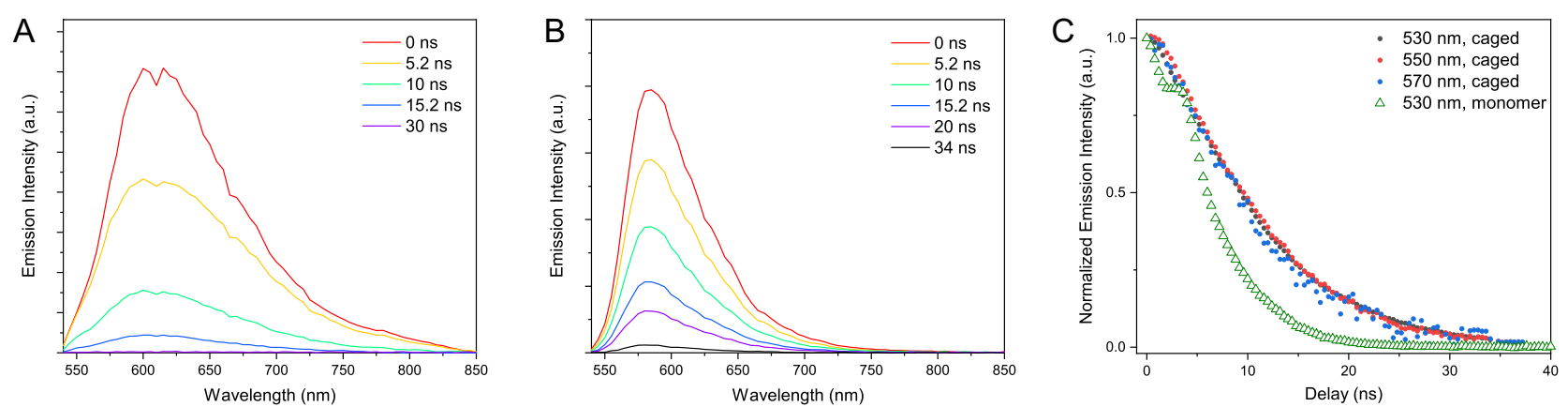

Figure S64. (A) Time-resolved fluorescence spectra of 4 dissolved in $\mathrm{MeCN}$. (B) Time-resolved fluorescence spectra of $\mathbf{4}_{2} \subset \mathbf{1}$ in $\mathrm{H}_{2} \mathrm{O}$. (C) Decay of fluorescence of $\mathbf{4}$ and $\mathbf{4}_{2} \subset \mathbf{1}$ at three different wavelengths. 
For nanosecond-to-microsecond transient absorption measurements on $\mathbf{2}$ and $\mathbf{2}_{2} \subset \mathbf{1}$, the same excitation beam as described on p. S40 was used in combination with an EOS-Sub-Nanosecond Transient Absorption Spectrometer (Ultrafast Systems, USA). The measurement was based on combining the ultrashort pump pulse with a white-light continuum generated by a photonic crystal fiber, thereby allowing for longer delays that are electronically triggered rather than using an optical delay line.
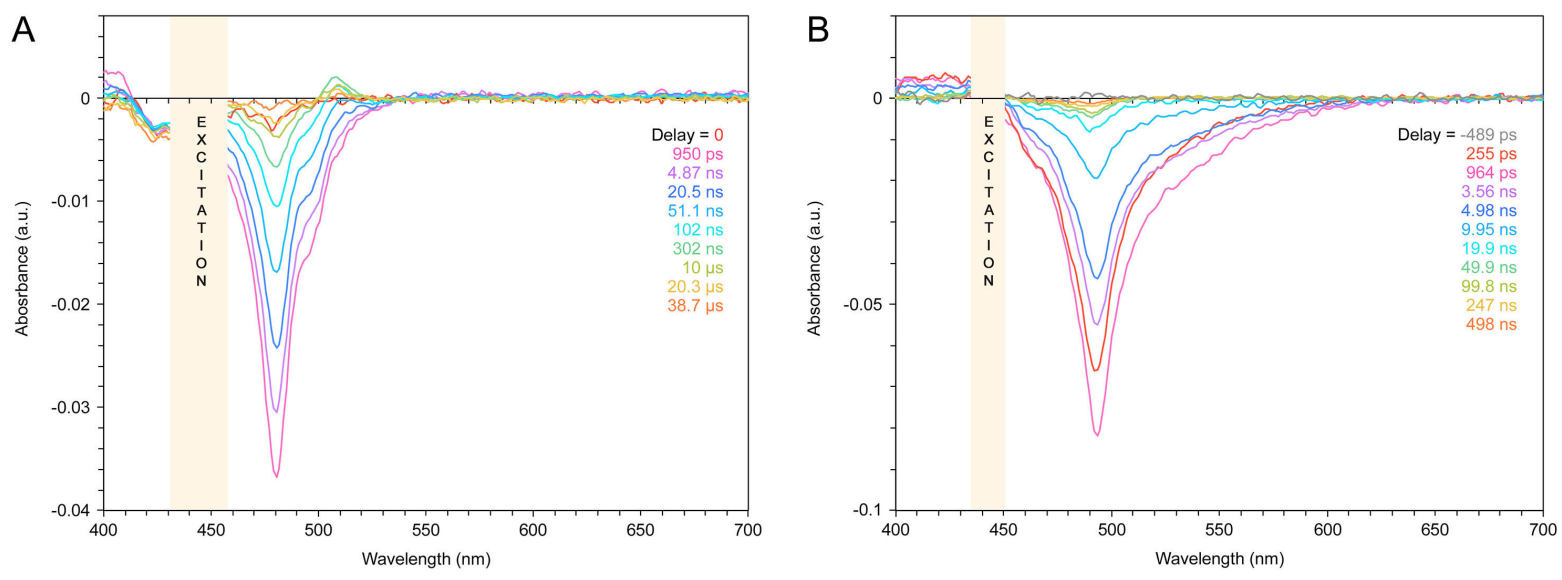

Figure S65. (A) Time-resolved absorption spectra of $\mathbf{2}_{2} \subset \mathbf{1}$ in $\mathrm{H}_{2} \mathrm{O}$ (replotted from Figure $2 \mathrm{c}$ in the main text). (B) Time-resolved absorption spectra of 2 in MeCN.

\section{Competitive binding of BODIPY 2 vs azobenzene guests}

We have previously demonstrated ${ }^{1}$ that azobenzene (Azo in Figure S66) and tetra-o-fluoroazobenzene (F-Azo in Figure S66) can bind within the cavity of cage 1. Moreover, we roughly estimated the association constant of Azo, $K_{\mathrm{a}}=\left[\mathrm{Azo}_{2} \subset 1\right] /[1] \cdot[\mathrm{Azo}]^{2}$, as $\sim 10^{9} \mathrm{M}^{-2}$. Although determining the $K_{\mathrm{a}}$ of F-Azo was not possible, we hypothesized that the binding strength would be higher owing to the presence $\mathrm{C}-\mathrm{F} \cdots \mathrm{H}$ hydrogen bond interactions. ${ }^{1}$ In order to obtain hints about the binding strength of the model BODIPY 2, we followed competitive binding of $\mathbf{2}$ vs Azo and $\mathbf{2}$ vs F-Azo in the presence of an equimolar amount of both guests with respect to cage 1 and in the presence of a three-fold excess of both guests with respect to 1 . The blue trace in Figure S66A shows the UV-vis spectrum of an equilibrated mixture of cage 1, BODIPY 2, and Azo in a 1:1:1 molar ratio. When the amount of both guests was increased three times, absorbance at $\sim 480 \mathrm{~nm}$ (due to encapsulated 2) increased slightly, whereas absorbance at $320 \mathrm{~nm}$ (due to encapsulated Azo) decreased (Figure S66A, red trace). This result indicates that in the presence of competition between 2 and Azo, BODIPY 2 is bound preferentially, i.e., its $K_{\mathrm{a}}$ is higher than $10^{9} \mathrm{M}^{-2}$. In contrast, when the amounts of 2 and F-Azo were increased from 1 equiv to 3 equiv with respect to the cage, we observed a drastic decrease in the amount of encapsulated 2 (blue and red spectra in Figure S66B, respectively), indicating that F-azo is bound significantly stronger than BODIPY 2. 

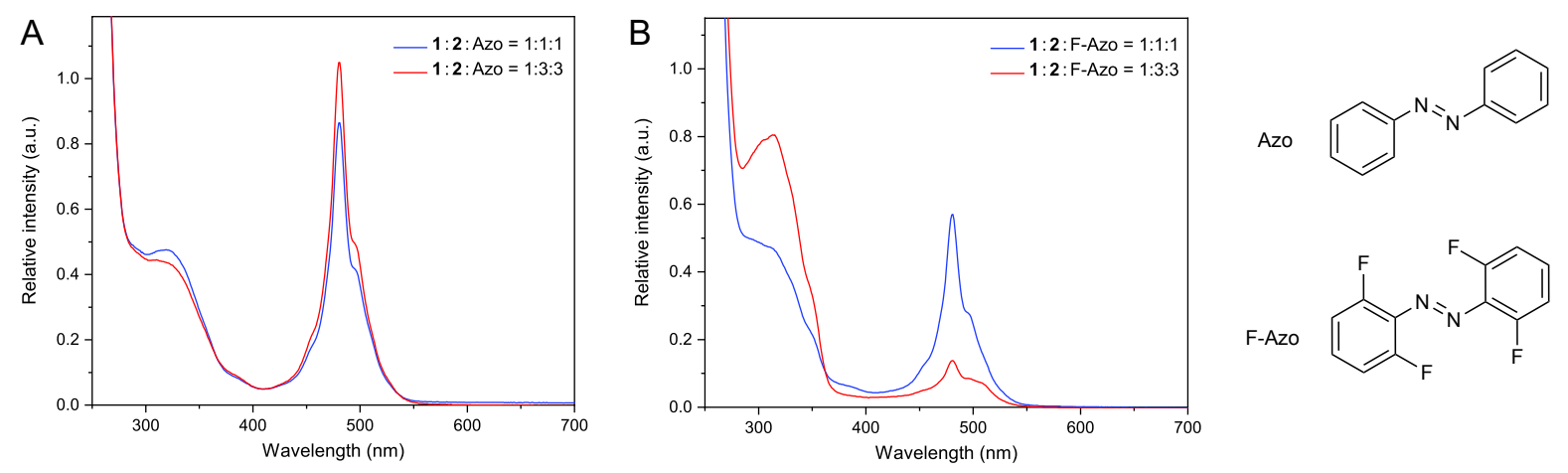

Figure S66. (A) UV-vis absorption spectra of cage 1 equilibrated with 1 equiv of BODIPY 2 and 1 equiv of azobenzene (blue trace), and with 3 eqiuv of 2 and 3 equiv of azobenzene (red trace). (B) UV-vis absorption spectra of cage 1 equilibrated with 1 equiv of 2 and 1 equiv of tetra-o-fluoroazobenzene (blue trace), and with 3 equiv of $\mathbf{2}$ and 3 equiv of tetra-o-fluoroazobenzene (red trace).

\section{Reversible switching between H- and J-aggregates of BODIPY 4}

The addition of 4 in $\mathrm{MeOH}(20 \mu \mathrm{L})$ to an excess $(980 \mu \mathrm{L})$ of water leads to the formation of J-type aggregates. ${ }^{4}$ These aggregates have a sharp absorbance band at $>600 \mathrm{~nm}$ (the exact position, intensity, and shape of the band depend on the degree of aggregation) and a sharp emission band at $\sim 630 \mathrm{~nm}$ (the very small Stokes shift is characteristic of J-aggregates). When this solution of J-aggregates of $4(77 \mu \mathrm{M})$ was treated with an excess of cage $\mathbf{1}$ in water, the J-aggregates' absorbance band rapidly decreased as a result of the encapsulation of $\mathbf{4}$ within $\mathbf{1}$ as an H-type dimer.

Figure S67 shows the changes in the UV-vis spectra of 4 during titration with aliquots of $\mathbf{1}$. The band due to J-aggregates can no longer be seen after the addition of 0.5 equiv of $\mathbf{1}$, whereas the H-dimer band increases until $\sim 0.8$ equiv of 1 has been added, in agreement with the $\sim 60 \%$ encapsulation yield of 4 within 1 . The J-to$\mathrm{H}$-aggregate transition can also be followed by fluorescence spectroscopy, which showed a decrease in the $\sim 630 \mathrm{~nm}$ sharp emission band accompanied by a rapid increase in a broader, more intense band at $\sim 585 \mathrm{~nm}$ (Figure S68). Remarkably, the addition of 1 equiv of the cage broke the J-aggregates of 4 and induced the formation of $\mathbf{4}_{2} \subset \mathbf{1}$ within only $\sim 30 \mathrm{~s}$.

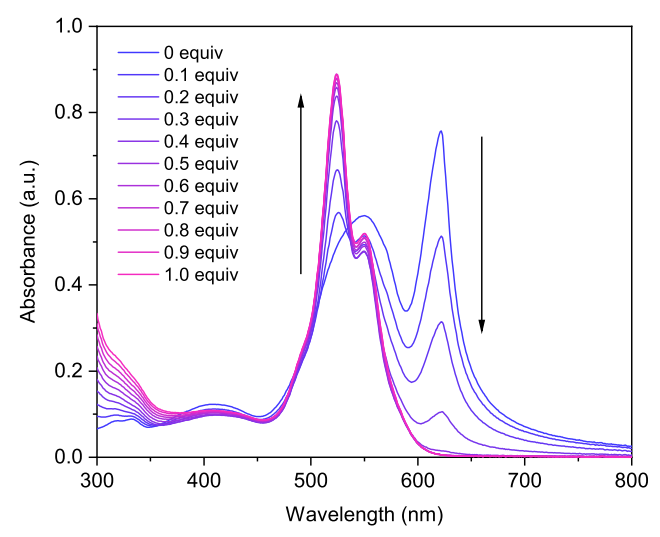

Figure S67. Titration of $\mathbf{4}$ aggregated in 1:49 MeOH: $\mathrm{H}_{2} \mathrm{O}(77 \mu \mathrm{M})$ with $\mathbf{1}$ to yield $\mathbf{4}_{2} \subset \mathbf{1}$. 


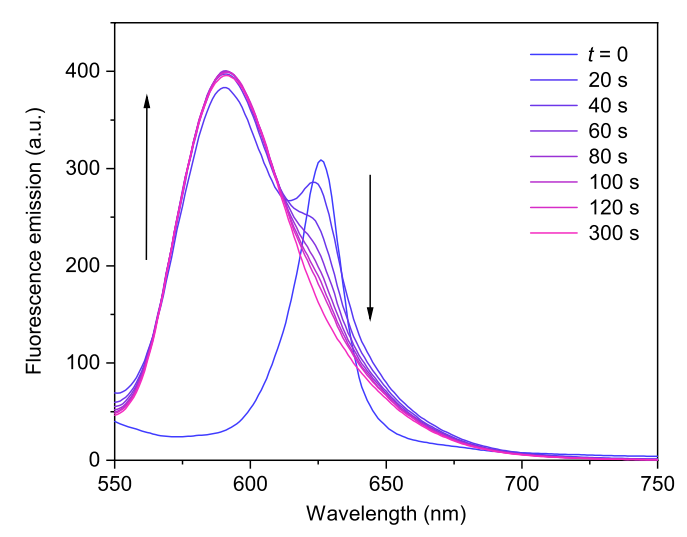

Figure S68. Changes in the fluorescence emission $\left(\lambda_{\mathrm{exc}}=530 \mathrm{~nm}\right)$ of $\mathbf{4}$ aggregated in 1:49 MeOH: $\mathrm{H}_{2} \mathrm{O}(39 \mu \mathrm{M})$ after adding 1 equiv of $\mathbf{1}$.

We hypothesized that the H-to-J-aggregate transition accompanying the encapsulation of $\mathbf{4}$ could be reversed by a controlled disassembly of cage $\mathbf{1}$, and that such disassembly could be achieved using aqueous KCN, which forms a strong complex with $\mathrm{Pd}^{2+}$. To verify that $\mathrm{KCN}$ can decompose the cage, a solution of $10 \mathrm{mg}$ ( 3.14 $\mu \mathrm{mol})$ of 1 in $0.7 \mathrm{~mL}$ of $\mathrm{D}_{2} \mathrm{O}$ in an NMR tube was treated with aliquots of $100 \mathrm{mM}$ aqueous $\left(\mathrm{D}_{2} \mathrm{O}\right) \mathrm{KCN}$. After the addition of each aliquot of $\mathrm{KCN}$ (which induced the precipitation of a white solid), the NMR tube was shaken and an NMR spectrum was recorded.

Cyanide $\mathrm{CN}^{-}$is a strong ligand for $\mathrm{Pd}^{2+}$; therefore, the addition of $\mathrm{KCN}$ gradually displaces the TIm $(1,3,5-$ triimidazoylbenzene, "triimidazole") and TMEDA ( $N, N, N^{\prime}, N^{\prime}$-tetramethylethylenediamine) ligands from the $\mathrm{Pd}^{2+}$ centers, resulting ultimately in the formation of the $\left[\mathrm{Pd}(\mathrm{CN})_{4}\right]^{2-}$ complex. Interestingly, the addition of 1.0 equiv of $\mathrm{KCN}$ (with respect to the $\mathrm{Pd}^{2+}$ centers) is sufficient to remove all the TIm ligands from the solution (TIm's aromatic protons are no longer detectable; Figure S69). Further addition of KCN replaces the bidentate TMEDA ligands; the NMR spectrum after the addition of 4.0 equiv corresponds to that of free TMEDA in water (two singlets at $2.55 \mathrm{ppm}$ and $2.26 \mathrm{ppm}$, corresponding to TMEDA's $\mathrm{CH}_{2}$ and $\mathrm{CH}_{3}$ groups; Figure S69). 


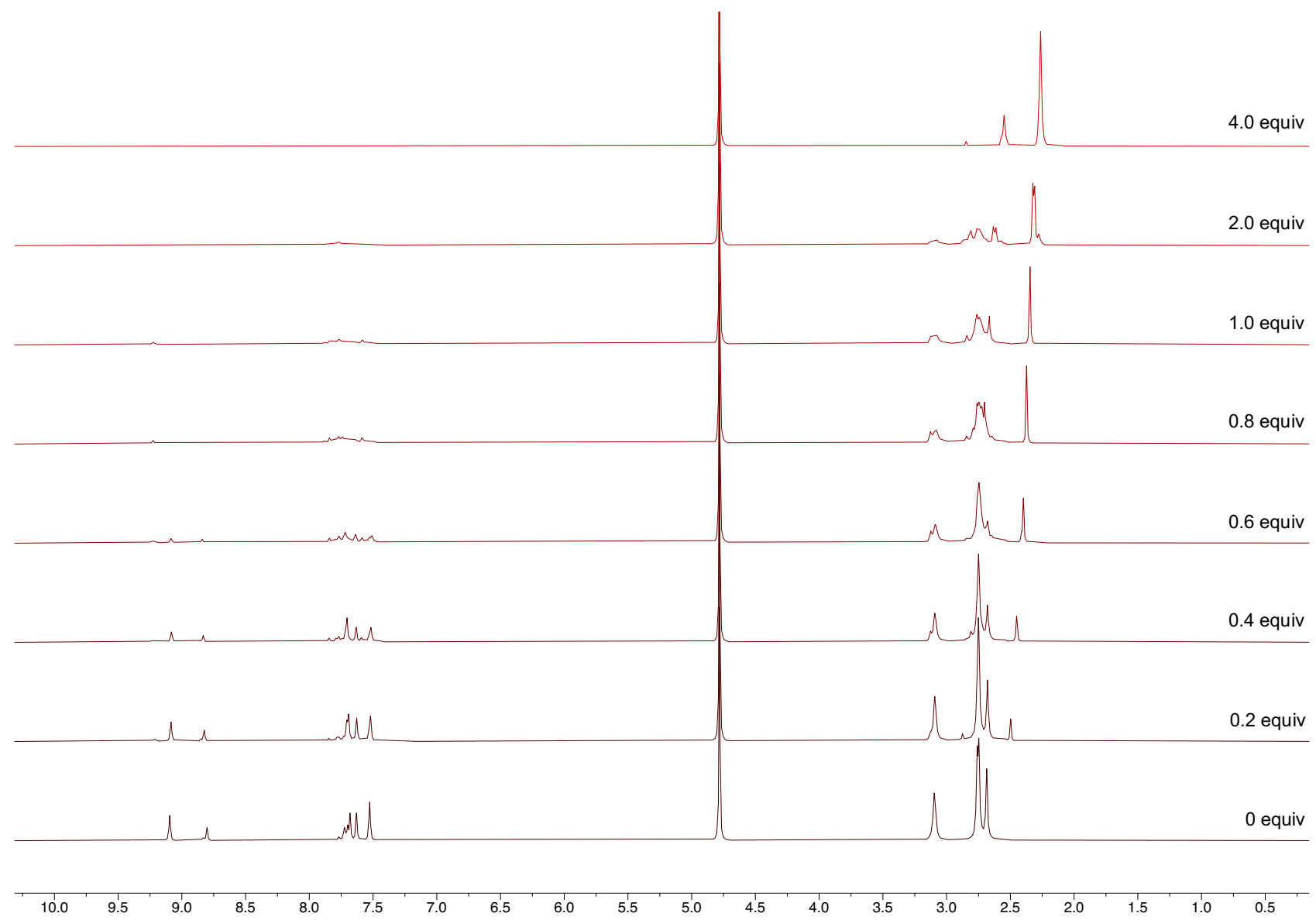

Figure S69. Changes in the ${ }^{1} \mathrm{H}$ NMR spectra of 1 during the gradual addition of $\mathrm{KCN}\left(400 \mathrm{MHz}, \mathrm{D}_{2} \mathrm{O}, 298\right.$ $\mathrm{K}$ ) (the numbers of equivalents of $\mathrm{KCN}$ with respect to the $\mathrm{Pd}^{2+}$ centers).

Having established that KCN can readily disassemble cage $\mathbf{1}$, we studied its effect on inclusion complex $\mathbf{4}_{2} \subset \mathbf{1}$. When a solution of $\mathbf{4}_{2} \subset \mathbf{1}$ in water was titrated with aqueous $\mathrm{KCN}$, the formation of J-aggregates of $\mathbf{4}$ was observed by UV-vis spectroscopy (see Figure S70). Notably, a substoichiometric amount of KCN was sufficient to restore the full intensity of the J-aggregate band. This process could also be followed by fluorescence spectroscopy (see Figure S71).

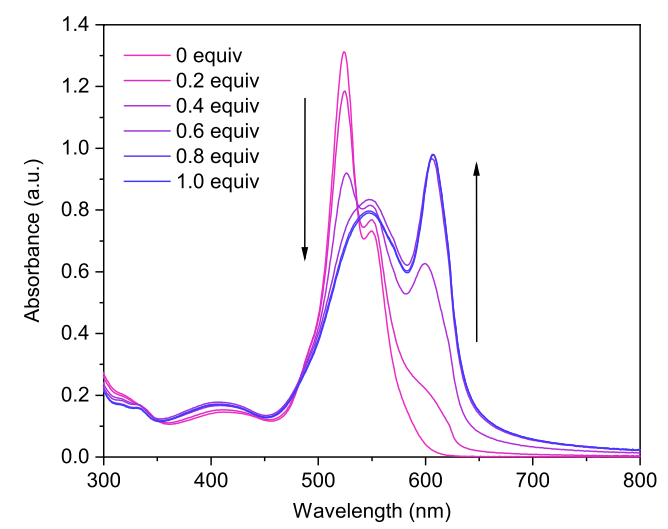

Figure S70. Changes in the absorption spectrum of $\mathbf{4}_{2} \subset \mathbf{1}$ in $\mathrm{H}_{2} \mathrm{O}(75 \mu \mathrm{M})$ during titration with $\mathrm{KCN}$ (the number of equivalents with respect to $\mathrm{Pd}^{2+}$ ). Note the non-monotonous change in the absorbance at $\lambda=548$ $\mathrm{nm}$ (corresponding to the wavelength of the maximum absorption of free $4 \mathrm{in} \mathrm{MeCN}$ ), which increases from $\sim 0.73$ (at $t=0$ ) to $\sim 0.83$ (for 0.6 equiv $\mathrm{KCN}$ ) and then decreases to $\sim 0.79$ (for 1.0 equiv $\mathrm{KCN}$ ). This result strongly suggests the presence of free $\mathbf{4}$ as an intermediate between the H-aggregate $\mathbf{4}_{2} \subset \mathbf{1}$ and the J-aggregate $\mathbf{4}_{\infty}$. 


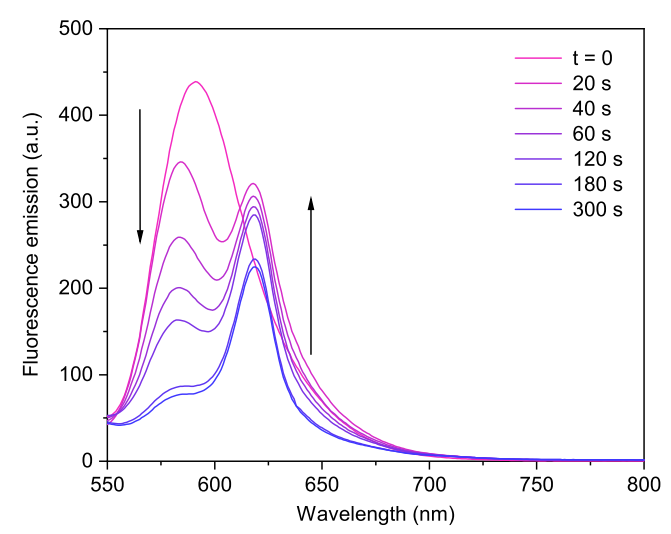

Figure S71. Changes in the fluorescence spectrum $\left(\lambda_{\mathrm{exc}}=530 \mathrm{~nm}\right)$ of $\mathbf{4}_{2} \subset \mathbf{1}$ in $\mathrm{H}_{2} \mathrm{O}(39 \mu \mathrm{M})$ following the injection of $\mathrm{KCN}\left(0.8\right.$ equiv with respect to $\left.\mathrm{Pd}^{2+}\right)$ followed over time.

Finally, we hypothesized that the pronounced color change caused by treating $\mathbf{4}_{2} \subset \mathbf{1}$ with KCN could be used to detect the presence of cyanide in water. To demonstrate a proof-of-concept system for cyanide detection, we soaked small pieces of agarose gels with aqueous $\mathbf{4}_{2} \subset \mathbf{1}$ and treated them with $\mathrm{KCN}$, among other inorganic salts (see Figure S72). We found that the only other salt causing visual changes similar to those induced by cyanide was thiocyanate, although it took significantly longer for color change to appear.
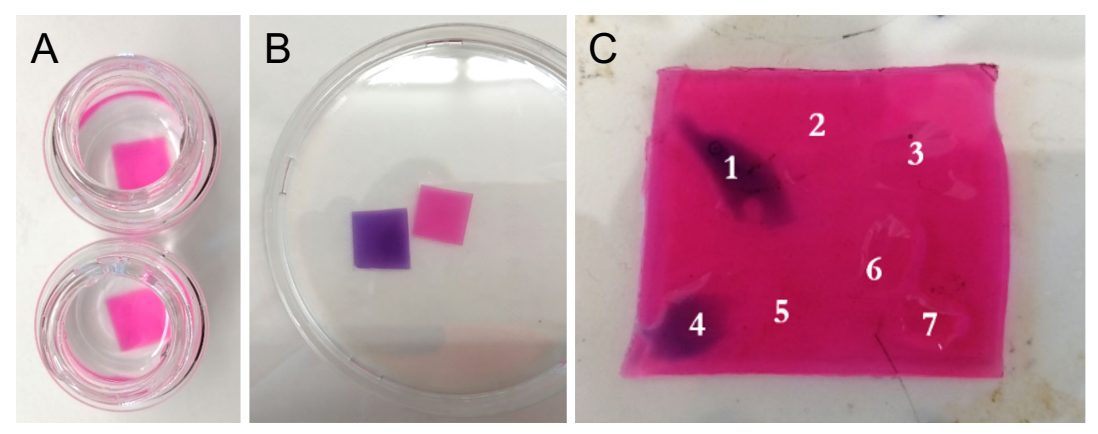

Figure S72. (A) Two pieces of agarose gel $\left(10 \times 10 \times 1 \mathrm{~mm}\right.$; prepared using $1 \mathrm{~g}$ of agarose and $50 \mathrm{~mL}$ of $\left.\mathrm{H}_{2} \mathrm{O}\right)$ soaked with aqueous $\mathbf{4}_{2} \subset \mathbf{1}(2 \mathrm{~mL} ; 0.5 \mu \mathrm{M})$. (B) Left: a $10 \times 10 \times 1 \mathrm{~mm}$ piece of agarose gel soaked with aqueous $\mathbf{4}_{2} \subset 1(2 \mathrm{~mL} ; 0.5 \mu \mathrm{M})$ following immersion in a $760 \mu \mathrm{M} \mathrm{KCN}$ solution $(2 \mathrm{~mL})$ for $10 \mathrm{~min}$. Right: a control sample not exposed to KCN. (C) A larger $(60 \times 50 \times 1 \mathrm{~mm})$ piece of agarose gel soaked with a $0.5 \mu \mathrm{M} \mathbf{4}_{2} \subset \mathbf{1}$ and exposed to different salts (150 nmol each): $\mathrm{KCN}$ (1), $\mathrm{KCl}$ (2), $\mathrm{NaI}$ (3), $\mathrm{NaSCN}$ (4), $\mathrm{Na}_{2} \mathrm{SO}_{4}$ (5), $\mathrm{NaNO}_{3}$ (6), and $\mathrm{AcOK}$ (7). The photograph was taken 10 min after applying droplets of the salt solutions. For KCN, the color change was immediate; for $\mathrm{NaSCN}$, it took several minutes. We have also tested $\mathrm{HCl}, \mathrm{Na}_{2} \mathrm{SO}_{3}$, and $\mathrm{NaOH}(10 \mu \mathrm{L}$ of $100 \mathrm{mM}$ solutions, corresponding to $1 \mu \mathrm{mol}) ; \mathrm{HCl}$ and $\mathrm{Na}_{2} \mathrm{SO}_{3}$ did not cause any visual changes; $\mathrm{NaOH}$ induced the decomposition of BODIPY and rendered the gel colorless. 


\section{X-ray data collection and structure refinement}

Single crystals of inclusion complexes $\mathbf{2}_{2} \subset \mathbf{1}, \mathbf{3}_{2} \subset \mathbf{1}$, and $\mathbf{4}_{2} \subset \mathbf{1}$ were obtained by slow evaporation of water from their respective aqueous solutions. Single crystals of $\mathbf{3}$ were obtained by slow evaporation of $\mathrm{CH}_{2} \mathrm{Cl}_{2}$ from a $\mathrm{CH}_{2} \mathrm{Cl}_{2}$ /hexane solution mixture of 3. All crystals were coated in Paratone oil (Hampton Research) and mounted on MiTeGen loops. They were flash frozen in a liquid nitrogen stream of the Oxford Cryostream system. Data collection was performed under a stream of nitrogen at $100 \mathrm{~K}$. The diffraction data of $\mathbf{2}_{2} \subset \mathbf{1}$ and $\mathbf{4}_{2} \subset \mathbf{1}$ were collected on a Rigaku XtaLAB ${ }^{\mathrm{PRO}}$ diffractometer using $\mathrm{Cu}-\mathrm{K} \alpha$ radiation $(1.54184 \AA)$ and processed with CrysAlisPRO. The diffraction data of $\mathbf{3}$ and $\mathbf{3}_{2} \subset \mathbf{1}$ were collected on a Bruker APEX-II Kappa CCD diffractometer using Mo-K $\alpha$ radiation $(0.7107 \AA$ ) and processed with Bruker SAINT. The structures were solved by direct methods using SHELXT. All non-hydrogen atoms were further refined by SHELXL with anisotropic displacement coefficients. Hydrogen atoms were placed in calculated positions and assigned isotropic displacement coefficients, $\mathrm{U}(\mathrm{H})=1.2 \mathrm{U}(\mathrm{C})$ or $1.5 \mathrm{U}$ (C-methyl), and their coordinates were refined in riding mode. The SQUEEZE protocol of Platon was run on $\mathbf{2}_{2} \subset \mathbf{1}, \mathbf{4}_{2} \subset \mathbf{1}$, and $\mathbf{3}_{2} \subset \mathbf{1}$. The crystal data and structural refinement are summarized in Table S3.

\begin{tabular}{|c|c|c|c|c|}
\hline Species & 3 & $2 \mathbf{2}_{2} \subset \mathbf{1}$ & $\mathbf{3}_{2} \subset \mathbf{1}$ & $\mathbf{4}_{2} \subset 1$ \\
\hline CCDC No. & 1990914 & 1551436 & 1990915 & 1990916 \\
\hline Formula* & $\mathrm{C}_{16} \mathrm{H}_{19} \mathrm{BF}_{2} \mathrm{~N}_{2} \mathrm{O}$ & $\begin{array}{c}\mathrm{C}_{124} \mathrm{H}_{178} \mathrm{~B}_{2} \mathrm{~F}_{4} \mathrm{~N}_{51.34} \\
\mathrm{O}_{36.73} \mathrm{Pd}_{6}\end{array}$ & $\begin{array}{c}\mathrm{C}_{256} \mathrm{H}_{360} \mathrm{~B}_{4} \mathrm{~F}_{8} \mathrm{~N}_{96} \\
\mathrm{O}_{79} \mathrm{Pd}_{12}\end{array}$ & $\begin{array}{c}\mathrm{C}_{124} \mathrm{H}_{172} \mathrm{~B}_{2} \mathrm{~F}_{10} \mathrm{~N}_{51} \\
\mathrm{O}_{50} \mathrm{Pd}_{6}\end{array}$ \\
\hline Formula weight ${ }^{\star}$ & 304.14 & 3711.68 & 7518.46 & 4027.16 \\
\hline Crystal system & Monoclinic & Monoclinic & Triclinic & Monoclinic \\
\hline Space group & $P 2_{1} / \mathrm{c}$ & $P 2{ }_{1}$ & $P-1$ & $P 2{ }_{1} / \mathrm{c}$ \\
\hline Crystal size (mm) & $0.20 \times 0.20 \times 0.20$ & $0.29 \times 0.28 \times 0.10$ & $0.23 \times 0.13 \times 0.04$ & $0.06 \times 0.04 \times 0.03$ \\
\hline Crystal color and shape & Purple prism & Red prism & Red plate & Purple plate \\
\hline Temperature (K) & 100 & 100 & 100 & 100 \\
\hline Wavelength ( $(\AA)$ & 0.71073 & 1.54184 & 0.71073 & 1.54184 \\
\hline a $(\AA)$ & $16.6385(9)$ & $15.0428(1)$ & $18.784(2)$ & $14.93362(6)$ \\
\hline b $(\AA)$ & $12.1506(6)$ & $41.8036(2)$ & $20.018(2)$ & $42.0135(2)$ \\
\hline c $(\AA)$ & $14.9855(8)$ & $15.4289(1)$ & $29.223(2)$ & $15.34383(7)$ \\
\hline$\alpha\left({ }^{\circ}\right)$ & 90 & 90 & $72.801(4)$ & 90 \\
\hline$\beta\left({ }^{\circ}\right)$ & $103.585(2)$ & $95.093(1)$ & $73.439(4)$ & 94.5872(4) \\
\hline$\gamma\left({ }^{\circ}\right)$ & 90 & 90 & $65.094(4)$ & 90 \\
\hline Volume $\left(\AA^{3}\right)$ & 2944.9(3) & 9664.1(1) & 7518.42 & $9596.09(7)$ \\
\hline $\mathbf{Z}$ & 8 & 2 & 1 & 2 \\
\hline$\rho_{\text {calcd }}\left(\mathrm{g} \mathrm{cm}^{-1}\right)$ & 1.372 & 1.276 & 1.334 & 1.394 \\
\hline$\mu\left(\mathrm{mm}^{-1}\right)$ & 0.102 & 5.062 & 0.645 & 5.243 \\
\hline No. of reflections (unique) & $25986(5389)$ & $39555(20061)$ & $121838(34262)$ & $146699(18217)$ \\
\hline $\mathbf{R}_{\text {int }}$ & 0.0404 & 0.1016 & 0.097 & 0.0390 \\
\hline Completeness to $\theta(\%)$ & 99.8 & 99.0 & 99.3 & 99.8 \\
\hline $\begin{array}{c}\text { Data / restraints / } \\
\text { parameters }\end{array}$ & $5378 / 0 / 416$ & $\begin{array}{c}39275 / 1056 / \\
2151\end{array}$ & $34031 / 289 / 2240$ & $18187 / 111 / 1206$ \\
\hline Goodness-of-fit on $F^{2}$ & 1.053 & 1.061 & 0.918 & 1.020 \\
\hline $\begin{array}{c}\text { Final } R_{1} \text { and } w R_{2} \text { indices } \\
{[I>2 \sigma(I)]}\end{array}$ & $0.0601,0.1531$ & $0.0795,0.2192$ & $0.0718,0.1809$ & $0.0727,0.2056$ \\
\hline $\begin{array}{c}R_{1} \text { and } w R_{2} \text { indices } \\
\text { (all data) }\end{array}$ & $0.0791,0.1687$ & $0.0829,0.2253$ & $0.1262,0.2040$ & $0.0747,0.2080$ \\
\hline
\end{tabular}

Table S3. Crystallographic data. * Derived from the crystal structure. 
1. Samanta, D.; Gemen, J.; Chu, Z.; Diskin-Posner, Y.; Shimon, L. J. W.; Klajn, R., Reversible photoswitching of encapsulated azobenzenes in water. Proc. Natl. Acad. Sci. USA 2018, 115, 9379-9384.

2. Nepomnyashchii, A. B.; Bröring, M.; Ahrens, J.; Bard, A. J., Synthesis, Photophysical, Electrochemical, and Electrogenerated Chemiluminescence Studies. Multiple Sequential Electron Transfers in BODIPY Monomers, Dimers, Trimers, and Polymer. J. Am. Chem. Soc. 2011, 133, 8633-8645.

3. Wang, H.; Vicente, M. G. H.; Fronczek, F. R.; Smith, K. M., Synthesis and Transformations of 5-Chloro2,2'-Dipyrrins and Their Boron Complexes, 8-Chloro-BODIPYs. Chem. Eur. J. 2014, 20, 5064-5074.

4. Choi, S.; Bouffard, J.; Kim, Y., Aggregation-induced emission enhancement of a meso-trifluoromethyl BODIPY via J-aggregation. Chem. Sci. 2014, 5, 751-755.

5. Kasha, M.; Rawls, H. R.; El-Bayoumi, M. A., The exciton model in molecular spectroscopy. Pure Appl. Chem. 1965, 11, 371-392. 